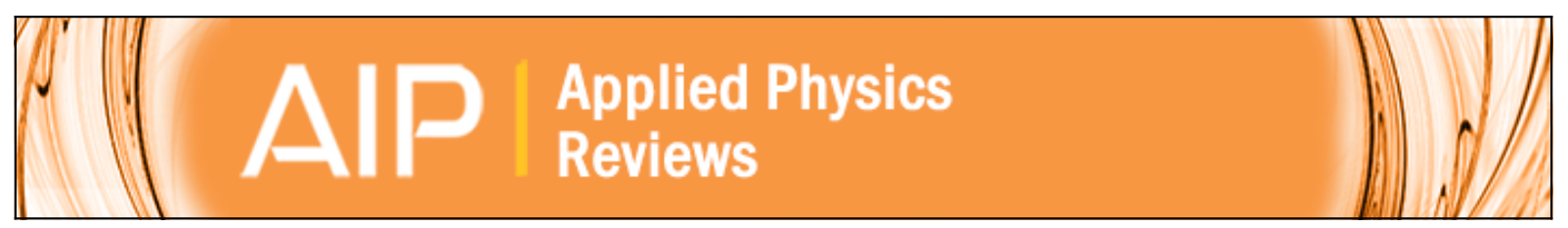

\title{
Intermediate band solar cells: Recent progress and future directions
}

Y. Okada, N. J. Ekins-Daukes, T. Kita, R. Tamaki, M. Yoshida, A. Pusch, O. Hess, C. C. Phillips, D. J. Farrell, K. Yoshida, N. Ahsan, Y. Shoji, T. Sogabe, and J.-F. Guillemoles

Citation: Applied Physics Reviews 2, 021302 (2015); doi: 10.1063/1.4916561

View online: http://dx.doi.org/10.1063/1.4916561

View Table of Contents: http://scitation.aip.org/content/aip/journal/apr2/2/2?ver=pdfcov

Published by the AIP Publishing

\section{Articles you may be interested in}

Proposal of high efficiency solar cells with closely stacked InAs/ln0.48Ga0.52P quantum dot superlattices:

Analysis of polarized absorption characteristics via intermediate-band

Appl. Phys. Lett. 105, 011120 (2014); 10.1063/1.4889805

Optimization of growth conditions of type-II $\mathrm{Zn}(\mathrm{Cd}) \mathrm{Te} / \mathrm{ZnCdSe}$ submonolayer quantum dot superlattices for intermediate band solar cells

J. Vac. Sci. Technol. B 31, 03C119 (2013); 10.1116/1.4797486

Influence of wetting layers and quantum dot size distribution on intermediate band formation in InAs/GaAs superlattices

J. Appl. Phys. 110, 073105 (2011); 10.1063/1.3631785

Quantum dot solar cell: Materials that produce two intermediate bands

J. Renewable Sustainable Energy 2, 013111 (2010); 10.1063/1.3327817

Emitter degradation in quantum dot intermediate band solar cells

Appl. Phys. Lett. 90, 233510 (2007); 10.1063/1.2747195

\section{A|P| $\left.\right|_{\text {Applied Physics }} ^{\text {Journal of }}$}

Journal of Applied Physics is pleased to announce André Anders as its new Editor-in-Chief 


\title{
Intermediate band solar cells: Recent progress and future directions
}

\author{
Y. Okada, ${ }^{1, a)}$ N. J. Ekins-Daukes, ${ }^{2, b)}$ T. Kita, ${ }^{3}$ R. Tamaki, ${ }^{1}$ M. Yoshida,${ }^{2}$ A. Pusch, ${ }^{2}$ O. Hess, ${ }^{2}$ \\ C. C. Phillips, ${ }^{2}$ D. J. Farrell, ${ }^{1, \mathrm{c})}$ K. Yoshida, ${ }^{1, \mathrm{~d})}$ N. Ahsan, ${ }^{1}$ Y. Shoji, ${ }^{1}$ T. Sogabe,${ }^{1}$ \\ and J.-F. Guillemoles ${ }^{4,5}$ \\ ${ }^{1}$ Research Center for Advanced Science and Technology (RCAST), The University of Tokyo, 4-6-1 Komaba, \\ Meguro-ku, Tokyo 153-8904, Japan \\ ${ }^{2}$ Department of Physics, Blackett Laboratory, Imperial College London, London SW7 2AZ, United Kingdom \\ ${ }^{3}$ Department of Electrical and Electronic Engineering, Graduate School of Engineering, Kobe University, \\ 1-1 Rokkodai, Nada, Kobe 657-8501, Japan \\ ${ }^{4}$ Institute of Research and Development of Energy from Photovoltaics (IRDEP-CNRS), Chatou 78401, France \\ ${ }^{5}$ NextPV, Joint RCAST-CNRS Laboratory, The University of Tokyo, 4-6-1 Komaba, Meguro-ku, \\ Tokyo 153-8904, Japan
}

(Received 1 November 2014; accepted 16 March 2015; published online 8 April 2015)

\begin{abstract}
Extensive literature and publications on intermediate band solar cells (IBSCs) are reviewed. A detailed discussion is given on the thermodynamics of solar energy conversion in IBSCs, the device physics, and the carrier dynamics processes with a particular emphasis on the two-step inter-subband absorption/recombination processes that are of paramount importance in a successful implementation high-efficiency IBSC. The experimental solar cell performance is further discussed, which has been recently demonstrated by using highly mismatched alloys and high-density quantum dot arrays and superlattice. IBSCs having widely different structures, materials, and spectral responses are also covered, as is the optimization of device parameters to achieve maximum performance. (C) 2015 AIP Publishing LLC. [http://dx.doi.org/10.1063/1.4916561]
\end{abstract}

\section{TABLE OF CONTENTS}

I. INTRODUCTION . ................ 1

A. Thermodynamic limits ............. 2

II. PHYSICS OF IBSCS ................ 3

A. Interband optical absorption strength...... 3

B. Photofilling and doping ........... 4

1. Development of IBSC device simulation. 4

2. Simulating the IBSC potential profiles .. 5

3. Simulating IBSC solar cell performance . 6

C. Interband recombination rates ......... 7

1. Radiative lifetime and doping ....... 7

2. Radiative lifetime and absorption coefficient................. 8

D. Factors limiting the efficiency of IBSC ... 8

1. Effect of carrier occupancy ......... 9

2. Influence of electron lifetime ....... 9

III. REVIEW OF PRESENT STATUS ON SOLAR CELL DEVELOPMENTS .............

\footnotetext{
a)Electronic mail: okada@mbe.rcast.u-tokyo.ac.jp. Also at NextPV, Joint RCAST-CNRS Laboratory, Meguro-ku, Tokyo, Japan.

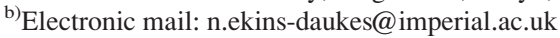

${ }^{c)}$ Currently at Grantham Institute - Climate Change and the Environment, Imperial College London, London SW7 2AZ, United Kingdom.

${ }^{\mathrm{d})}$ Currently at Institute of Applied Physics, University of Tsukuba, 1-1-1

Tennodai, Tsukuba, Ibaraki 305-8573, Japan.
}

A. Highly mismatched alloys .......... 10

1. HMA materials and electronic properties 10

2. III-V GaNAs-based thin-film IBSC ..... 13

3. II-VI ZnTeO-based thin-film IBSC ..... 18

B. Quantum dot arrays.............. 20

1. Fabrication techniques of multi-stacking QDs layers ................. 20

2. Characteristics of type-II QD structures. . 24

3. Effect of doping on QDSCs.......... 26

4. Concentrated photovoltaic (CPV) characterization ................. 29

C. IB materials with transition metals........ 33

1. Dilute magnetic semiconductors ....... 33

2. Lifetime recovery ............. 34

3. Ab-initio computation of IB and DMS band structure.............. 34

D. Spectroscopy measurements and techniques. 36

1. Steady state emission from an IBSC .... 36

2. Two-step photon absorption (TSPA) photocurrent spectroscopy ...........

3. Carrier dynamics of inter-subband transition in InAs/GaAs QDs ............

4. Carrier recombination process in InAs/ GaAs QD-IBSCs ................

5. Spectrally resolved inter-subband photocurrent spectroscopy ............. 43

IV. FUTURE DIRECTIONS ............ 44 


\section{INTRODUCTION}

The possibility for increasing the efficiency of photovoltaic solar cells via sequential absorption of photons in a single material has a relatively long history. Starting with the impurity photovoltaic solar cell in 1960, where sequential absorption was suggested to proceed via defect levels, ${ }^{1}$ the concept gained renewed interest with the proposal of the intermediate band solar cell (IBSC) ${ }^{2,3}$ in the mid-1990s. The compelling difference between the impurity band and the IB concept is that forming an IB should, in principle, reduce the nonradiative transition rate that is likely to dominate when carriers are localised onto isolated impurities. ${ }^{4-6}$ However, the principle challenge to establish an IB within a semiconductor material that supports strong optical transitions with relatively low nonradiative recombination in comparison to the rate of photo-generation by solar photons has remained. This requirement places some fundamental constraints on the nature of the IB that are discussed from a theoretical standpoint in Sec. II and some very significant demands on the semiconductor materials discussed in Sec. III.

\section{A. Thermodynamic limits}

By means of introduction to the IBSC, it is helpful to compare it against a conventional Shockley-Queisser (SQ) solar cell. The Shockley-Queisser efficiency limit ${ }^{7}$ places an upper bound on the power that can be converted from a single semiconductor junction, resulting in a maximum efficiency of $31 \%$ at 1 sun illumination. To achieve these efficiencies, it is assumed that all photons with energies in excess of the bandgap energy are absorbed, that carrier mobility is infinite enabling all carriers to be collected, and that all recombination is radiative. These ideal conditions can be approached by the very best solar cells; recently, a GaAs solar cell has been demonstrated with an efficiency of $28.8 \% .^{8}$ The fundamental losses in a SQ solar cell arise from a trade-off between carrier thermalisation and optical transparency that manifest themselves in a solar cell as a trade-off between current and voltage, respectively. ${ }^{9}$ This trade-off is evident from the current density-voltage $(J-V)$ curves from three SQ solar cells are shown in Fig. 1. A low-gap solar cell yields a high current but low voltage, yet a high-gap solar

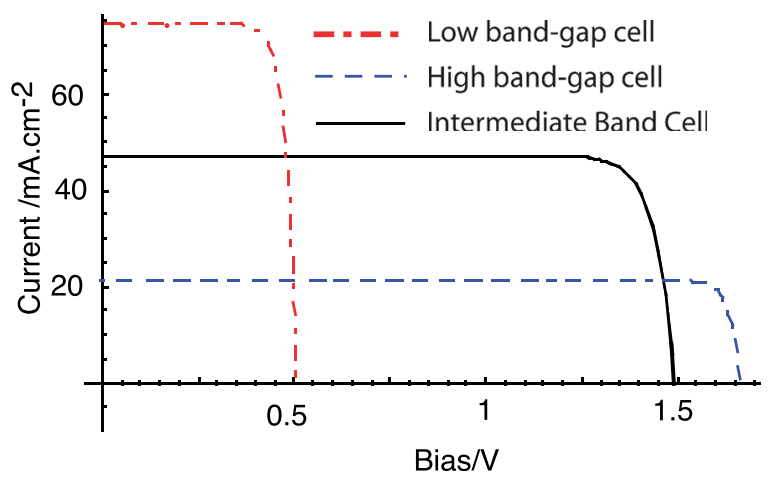

FIG. 1. $J-V$ curves corresponding to two SQ solar cells with absorption thresholds corresponding to the lowest and highest energy gaps in an IBSC and a third $J-V$ curve corresponding to an IBSC with the same absorption thresholds. cell yields a low current but high voltage. By virtue of the sequential absorption, an IBSC can maintain the broad absorption profile of a low-gap solar cell, while preserving much of the voltage of a high-gap cell, illustrated in Fig. 2. Despite the absorption profile of the IBSC being identical to the lowgap cell, the current is approximately half that of the lowgap SQ cell. This arises since the additional photocurrent over the high-gap SQ cell arises from sequential absorption where two photons to be absorbed for every electron in the external circuit.

In a conventional illuminated solar cell, the carrier populations in the conduction band (CB) and valence band (VB) can be considered to be in quasi-thermal equilibrium, meaning that thermal equilibrium exists independently within the electron population in the $\mathrm{CB}$ and hole population in the $\mathrm{VB}^{10}$ but not between the two bands since an electrochemical potential difference now separates the electrons in the CB and VB giving rise to the voltage of the solar cell. In an IBSC, the voltage preservation arises from establishing a third population of carriers in the quasi-thermal IB. The occupancy of all three bands can be described using a Fermi energy, establishing three electrochemical potential differences $\mu_{1,2,3}$ as shown in Fig. 2 such that $\mu_{1}=\mu_{2}+\mu_{3}$. Since electrical contacts are made only to the $\mathrm{CB}$ and $\mathrm{VB}$, the overall voltage of the solar cell is given by $\mu_{1}$. The voltage of an ideal IBSC is therefore considerably higher than that a SQ solar cell with an equivalent absorption profile. The ideal IBSC voltage is marginally lower than that what one would expect from a SQ solar cell with the same CB-VB bandgap since recombination from the CB-VB can proceed via $R_{\mathrm{IC}}$ and $R_{\mathrm{VI}}$, in addition to $R_{\mathrm{VC}}$. A limiting efficiency of $63.1 \%$ is obtained for the IBSC under the assumption of full concentration and a $6000 \mathrm{~K}$ blackbody approximation to sunlight. ${ }^{3}$ To illustrate the dependency of this limiting efficiency on the fundamental, host material bandgap, Fig. 3 shows the efficiency of an IBSC for both maximum concentration and 1 sun. The correspondence between the fundamental host bandgap and the intermediate bandgap is also shown. It is clear that relatively wide bandgap host materials are required for efficient operation of an IBSC. Higher efficiencies up to $65.1 \%$ and marginally different optimal threshold energies are found when considering terrestrial solar spectra. ${ }^{11}$

The IBSC concept shown in Fig. 2 represents the optimal configuration under maximum solar concentration. However, all practical solar cells will operate at lower solar concentrations and there is an efficiency advantage to be

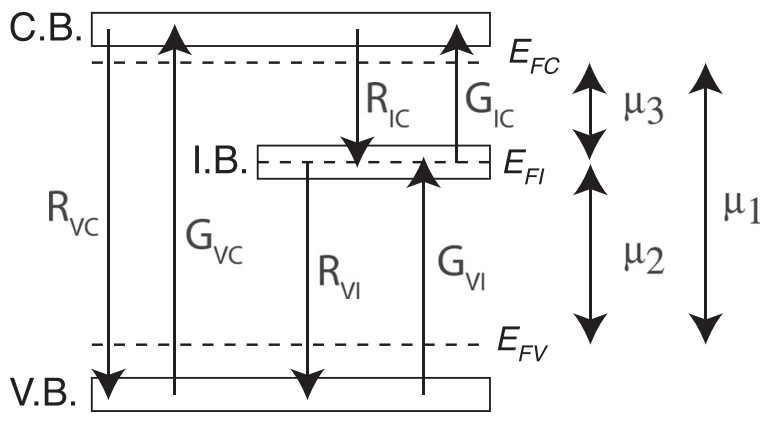

FIG. 2. Schematic diagram showing the basic transitions in an IBSC. 


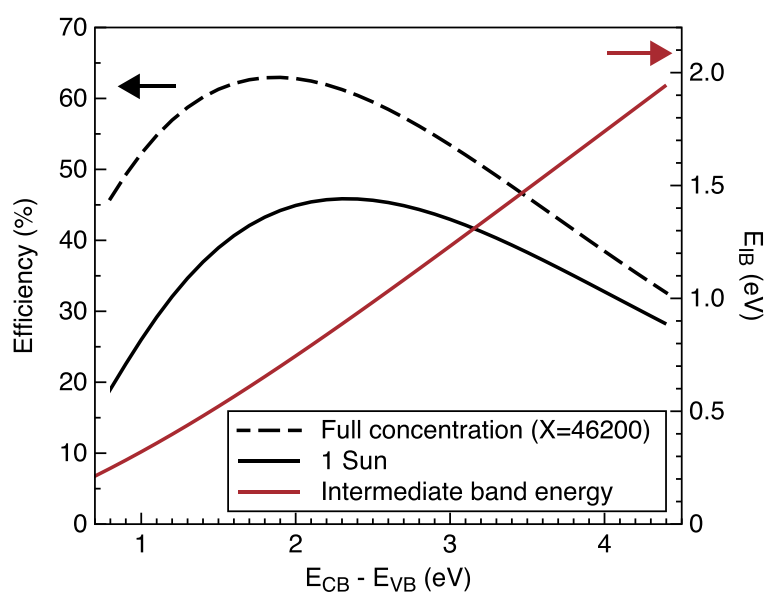

FIG. 3. Limiting efficiency of an IBSC calculated assuming $6000 \mathrm{~K}$ blackbody radiation from the sun. The calculation has been performed as a function of the widest bandgap (host) material and for maximum concentration as well as 1 sun conditions.

gained by introducing a relaxation stage within the IB shown in Fig. 4. Critically, optical transitions between the relaxed IB (RB) and VB are forbidden, hence the only route for relaxation via the IB now involves surmounting a potential barrier from the $\mathrm{RB}$ to the $\mathrm{VB}$. In this configuration, the 1 sun limiting efficiency for an ideal IBSC of $46.8 \%$ rises to $48.5 \%$ with a relaxation energy of $\Delta E=0.27 \mathrm{eV} .^{12}$ This counterintuitive result that introducing a loss results in a more efficient solar cell arises due to entropy generated upon recombination in solar cells with mismatched optical étendues. ${ }^{13}$ By lowering the overall recombination via the IB, this unnecessary entropy loss is reduced. At maximum concentration, the étendues of incident light and recombination are matched and hence the entropy loss is eliminated and with it the fundamental advantage of the relaxation stage. The fundamental efficiency benefit of relaxation has also been recognised in up converting systems that rely upon sequential absorption. ${ }^{14}$ A molecular system promoting this scheme has been proposed ${ }^{15}$ and a ferromagnetic approach ${ }^{16}$ discussed further in Sec. III C.

\section{PHYSICS OF IBSCs}

To move beyond the conceptual limiting efficiency analysis of the IBSC, it is necessary to consider the absorption and emission processes in more detail. In a conventional solar cell, the transitions are uniquely from a filled VB into

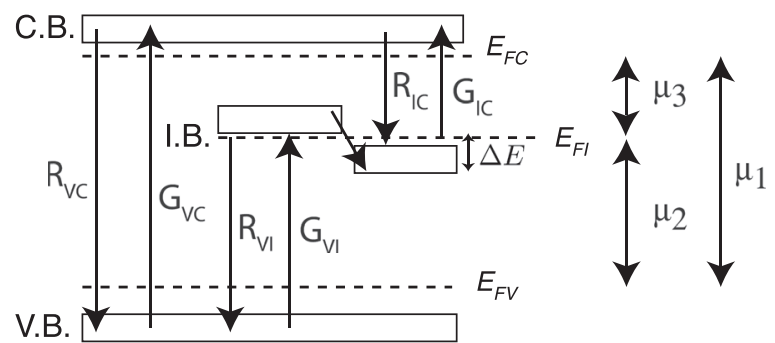

FIG. 4. Band diagram for an IBSC incorporating a split IB and a relaxation (ratchet) step $\Delta E$. Transitions from VB to IB are allowed as are transitions from IB to $\mathrm{CB}$, but the transition from $\mathrm{IB}$ to $\mathrm{VB}$ and $\mathrm{CB}$ to $\mathrm{IB}$ is suppressed owing to the occupancy of the IB. an empty CB. However, in an IBSC, the IB must support transitions both in-to and out-of the IB, thereby complicating the device analysis and practical implementations. In this section, the key theoretical results that elucidate the requirements for an efficient IBSC are reviewed.

\section{A. Interband optical absorption strength}

Photons of energy $\hbar \omega$ impinging on a material can stimulate both upwards (absorptive) and downwards (emissive) transitions. Therefore, the net absorption rate of photons, from the $\mathrm{VB}$ to the $\mathrm{CB}, W_{\mathrm{VC}}\left(\mathrm{cm}^{-3} \mathrm{~s}^{-1}\right)$, is given by the difference between the simulated absorption $r_{a}(\hbar \omega)$ and emission $r_{s t}(\hbar \omega)$ rates

$$
W_{\mathrm{VC}}(\hbar \omega)=r_{a}(\hbar \omega)-r_{s t}(\hbar \omega) .
$$

In turn, these rates are proportional to the photon density $n_{\gamma}(\hbar \omega)$ and to the integral over all possible combinations of occupied $n_{o}(\epsilon)$ and unoccupied $n_{u}(\epsilon)$ carrier populations which preserve both energy and momentum ${ }^{17}$

$$
\begin{aligned}
& r_{a}(\hbar \omega)=n_{\gamma}(\hbar \omega) \int_{0}^{\infty} M(\epsilon, \hbar \omega) n_{o}(\epsilon) n_{u}(\epsilon+\hbar \omega) d \epsilon, \\
& r_{s t}(\hbar \omega)=n_{\gamma}(\hbar \omega) \int_{0}^{\infty} M(\epsilon, \hbar \omega) n_{u}(\epsilon) n_{o}(\epsilon+\hbar \omega) d \epsilon .
\end{aligned}
$$

In the above, energy conservation is ensured by evaluation of the final state at energy $\epsilon+\hbar \omega$, and momentum conservation is ensured by the interband momentum matrix element $M(\hbar \omega)$ (which is only non-zero for combination of states which preserve momentum). The integration can be removed by utilising the one-to-one relationship between the photon energy $\hbar \omega$ and energy state $\epsilon\left(k^{\prime}\right)$ as shown in Fig. 5, where $k^{\prime}$ is given by

$$
k^{\prime}=\sqrt{\frac{2}{\hbar^{2}}\left(\frac{1}{m_{e}^{\star}}+\frac{1}{m_{h}^{\star}}\right)\left(\hbar \omega-E_{g}\right)},
$$

where $E_{g}$ is the bandgap energy and $m_{e}^{\star}$ and $m_{h}^{\star}$ are, respectively, the CB and VB effective masses.

Optical transitions in a semiconductor result in a negligible change in $k$, hence there is a unique correspondence between the photon energy and location in $k$-space for an

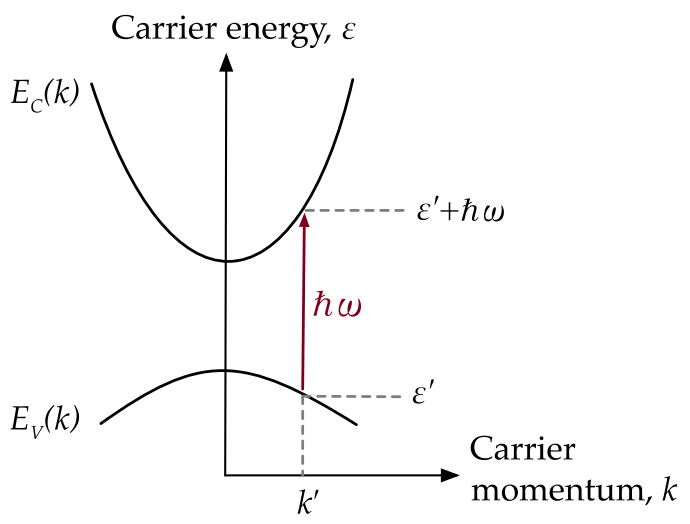

FIG. 5. Allowed optical transitions conserve energy and momentum. 
optical transition. For this reason, the integration in Eqs. (2) and (3) can be removed provided they are evaluated at $\epsilon\left(k^{\prime}\right)$

$$
\begin{aligned}
& r_{a}(\hbar \omega)=n_{\gamma}(\hbar \omega) M\left(\epsilon^{\prime}, \hbar \omega\right) n_{o}\left(\epsilon^{\prime}\right) n_{u}\left(\epsilon^{\prime}+\hbar \omega\right), \\
& r_{s t}(\hbar \omega)=n_{\gamma}(\hbar \omega) M\left(\epsilon^{\prime}, \hbar \omega\right) n_{u}\left(\epsilon^{\prime}\right) n_{o}\left(\epsilon^{\prime}+\hbar \omega\right),
\end{aligned}
$$

where we have used the shorthand notation $\epsilon\left(k^{\prime}\right)=\epsilon^{\prime}$.

Expressions for the occupied $n_{o}(\epsilon)$ and unoccupied $n_{u}(\epsilon)$ carrier populations at their respective energetic locations in the band are functions of the density of states of the lower band, $g_{\mathrm{V}}(\epsilon)$, and upper band, $g_{\mathrm{C}}(\epsilon)$, in combination with the Fermi-Dirac occupancy functions of $\mathrm{CB}$, $f_{\mathrm{C}}(\epsilon)$, and of $\mathrm{VB}, f_{\mathrm{V}}(\epsilon)$. Therefore, the net absorption rate is arrived at

$$
\begin{aligned}
W_{\mathrm{VC}}(\hbar \omega)= & n_{\gamma} M\left(\epsilon^{\prime}, \hbar \omega\right) g_{\mathrm{V}}\left(\epsilon^{\prime}\right) g_{\mathrm{C}}\left(\epsilon^{\prime}+\hbar \omega\right) \\
& \times\left(f_{\mathrm{V}}\left(\epsilon^{\prime}\right)-f_{\mathrm{C}}\left(\epsilon^{\prime}+\hbar \omega\right)\right) .
\end{aligned}
$$

The net absorption rate can also be defined using the BeerLambert law

$$
W_{\mathrm{VC}}(\hbar \omega)=-\frac{d I}{d x}=\alpha(\hbar \omega) I(\hbar \omega),
$$

where $I(\hbar \omega)=v_{g}(\hbar \omega) n_{\gamma}(\hbar \omega)$ is the photon flux $\left(\mathrm{cm}^{-2} \mathrm{~s}^{-1}\right)$ and group velocity in the material, $v_{g}(\hbar \omega)$, is calculated from the refractive index $n(\hbar \omega)$. Equating these two expressions, a relation for absorption coefficient which includes the effect of carrier occupancy is found to be

$$
\alpha(\hbar \omega)=\alpha_{0}(\hbar \omega)\left(f_{\mathrm{V}}\left(\epsilon^{\prime}\right)-f_{\mathrm{C}}\left(\epsilon^{\prime}+\hbar \omega\right)\right),
$$

where $\alpha_{0}(\hbar \omega)=M\left(\epsilon^{\prime}, \hbar \omega\right) g_{\mathrm{V}}\left(\epsilon^{\prime}\right) g_{\mathrm{C}}\left(\epsilon^{\prime}+\hbar \omega\right) / v_{g}(\hbar \omega)$ gives the maximum absorption coefficient of the material. This could be calculated using Fermi's golden rule or for most instances $\alpha_{0}(\hbar \omega)$ can simply be set equal to the experimental value of absorption coefficient (because usually $f_{\mathrm{V}}\left(\epsilon^{\prime}\right)$ $\left.-f_{\mathrm{C}}\left(\epsilon^{\prime}+\hbar \omega\right)=1\right)$. Therefore, the net absorption rate is given by

$$
W_{\mathrm{VC}}(\hbar \omega)=\alpha_{0}(\hbar \omega) I(\hbar \omega)\left(f_{\mathrm{V}}\left(\epsilon^{\prime}\right)-f_{\mathrm{C}}\left(\epsilon^{\prime}+\hbar \omega\right)\right) .
$$

This expression indicates that the interband transition rate can be in one of three regimes:

(1) $\left(f_{\mathrm{V}}\left(\epsilon^{\prime}\right)-f_{\mathrm{C}}\left(\epsilon^{\prime}+\hbar \omega\right)\right) \sim 1$, interband absorption rate is strong: this is the usual property for semiconductor in which the VB is full and the CB is empty.

(2) $\left(f_{\mathrm{V}}\left(\epsilon^{\prime}\right)-f_{\mathrm{C}}\left(\epsilon^{\prime}+\hbar \omega\right)\right) \sim 0$, interband absorption rate is zero: indicating the transition has been bleached due to high occupancy in both levels.

(3) $\left(f_{\mathrm{V}}\left(\epsilon^{\prime}\right)-f_{\mathrm{C}}\left(\epsilon^{\prime}+\hbar \omega\right)\right) \sim-1$, interband absorption rate is negative: indicating the semiconductor is behaving as an optical gain medium.

All conventional solar cells operate in the first regime, where the occupancy factor is normally ignored since the ground state can be assumed to be filled and empty excited states always available. However, for an IBSC the situation is more complex since the IB must support optical transitions both in-to and out-of the same band.
The same optical properties desired in the IBSC already exist in multi-junction solar cells. We may ask why all optical transitions in multi-junction solar cells are strong and yet observing the same transitions in an IB is so challenging. Figure 6 shows a multi-junction solar cell along with band diagrams and the occupancy indicated by the equilibrium Fermi level $\left(E_{F}\right)$ and quasi-Fermi levels $\left(E_{F_{\mathrm{V}}}\right.$ and $\left.E_{F_{\mathrm{C}}}\right)$. In Fig. 6, the Fermi-level, in both junctions, is well above the VB and also well below the CB; therefore the optical absorption is strong. When illuminated additional electrons and holes are generated, yet the intra-band relaxation rates are very much faster than any inter-band recombination rate, so two quasi-Fermi levels can be defined $E_{F_{\mathrm{V}}}$ and $E_{F_{\mathrm{C}}}$ for the $\mathrm{VB}$ and $\mathrm{CB}$, respectively. ${ }^{10}$ Under typical solar illumination levels, the quasi-Fermi levels remain well below the band edges and the optical absorption remains strong.

Figure 7 shows the optical transitions via an IB (the third wide gap junction is not drawn) and possible Fermilevel arrangements for an IBSC. In Fig. 7(a), the transition rate from IB to $\mathrm{CB}$ is severely limited because of the low occupancy in the IB. In the equivalent circuit model, the two subcells are series connected; therefore, the cell suffers from very poor current matching between these two junctions, resulting in very low efficiency. Figure 7(b) shows a more ideal situation where the IB is approaching the "half filled condition."3

\section{B. Photofilling and doping}

The basic principles of the IBSC are well described by fundamental detailed balance models ${ }^{3}$ but for a more practical understanding of IBSC, it is necessary to consider a more realistic model.

\section{Development of IBSC device simulation}

In a detailed solar cell device simulation, the material properties and carrier transport need to be accurately simulated. Under steady state operation, there are two basic equations; the Poisson and continuity equation. The Poisson equation determines the electrostatic potential $\psi$ depending on the charge density $\rho$

$$
\nabla \cdot(\varepsilon \nabla \psi)=-\rho,
$$

where $\varepsilon$ is dielectric constant, and the charge density usually contains free carriers, ionized impurities and trapped charges. The continuity equation is given by

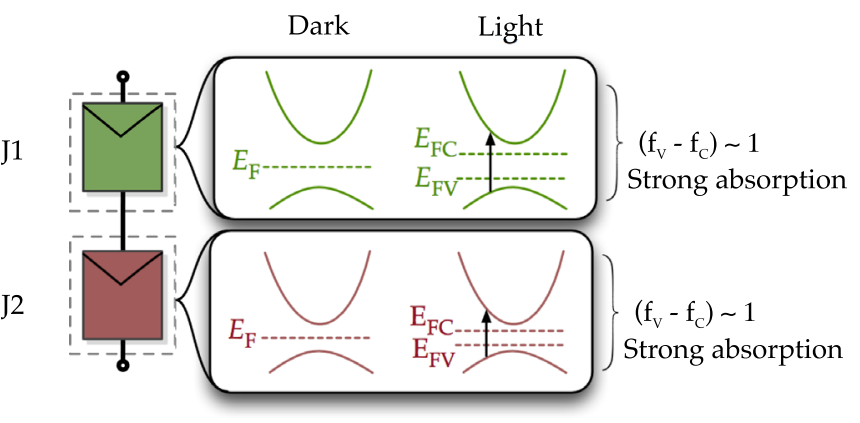

FIG. 6. Strong optical transitions in multi-junction solar cells. 


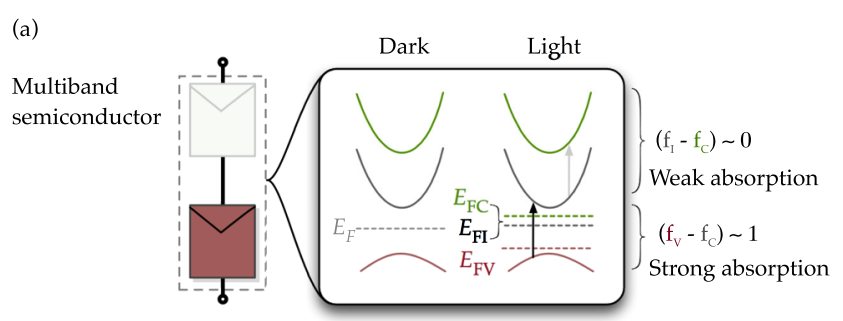

(b)

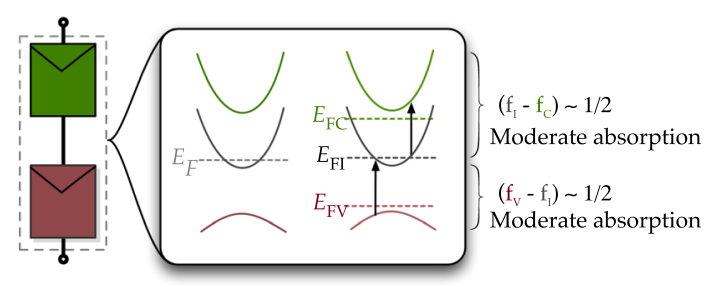

FIG. 7. Two possible regimes of operation for an IB material: (a) below the degeneracy limit in which the IB to CB absorption is weak, (b) approaching degeneracy in which the transition rates in-to and out-of the IB are balanced.

$$
\begin{gathered}
\nabla \cdot \mathbf{J}_{e}=q R, \\
\nabla \cdot \mathbf{J}_{h}=-q R,
\end{gathered}
$$

where $\mathbf{J}_{e}$ and $\mathbf{J}_{h}$ are electron and hole currents, respectively, and $q$ and $R$ are elementary charge and net recombination rate. In drift-diffusion method, the current densities are given by the drift-diffusion equation.

Solar cell device characteristics can be determined by self-consistently solving the Poisson equation, the carrier continuity equation with drift-diffusion equation in steady state. Compared with the conventional single-junction solar cells, the IBSC introduces additional states within the bandgap and these states can become charged with trapped or free carriers. These trapping effects require two additional considerations: how carriers are trapped or extracted from the IB and the effect of their charge on the electrostatic potential profile.

For IBSC device simulation, the transport equation is usually treated within the classical, i.e., drift-diffusion equation. Compared to the semi-classical and quantum methods, ${ }^{18}$ the drift-diffusion method has an advantage that it can include whole cell structure which scale is $\sim 1 \mu \mathrm{m}$. This approach captures all the relevant recombination processes, both in the IB region but also elsewhere in the device structure, e.g., surface recombination. In addition, the potential profile in the IB region and the carrier extraction efficiency from IB largely depend on doping concentration in the device. Therefore, a drift-diffusion calculation can provide intrinsic device properties of IBSC device operation.

In 2002, Martí et al. studied spatial dependence of carrier density and current elements using a quasi-drift diffusion model. ${ }^{19}$ This model is only valid when the quasi-chargeneutrality condition is satisfied. However, this work only included the IB active region. In 2009, Lin and Phillips ${ }^{20}$ developed the drift-diffusion simulation without IB occupancy dependence of optical carrier generation rate through IB. This simulation accounted for the whole device structure, and clarified how quasi-Fermi levels are connected throughout the device. They showed how the the potential profile is modified under short-circuit condition from its equilibrium value due to optical filling of the IB. In 2010, Yoshida et $a .^{21}$ developed IBSC drift-diffusion simulation where IB occupancy is also solved self-consistently accounting for the generation and recombination rates via IB and the potential profile. They showed how the need for doping arises in the IB at low solar concentrations. ${ }^{22}$ Strandberg and Reenaas ${ }^{23}$ compared the effects of pre-filling (doping) and photofilling for IB region using similar treatment used in Ref. 19. While many works only include radiative recombination process, Tobías et al., also developed IBSC drift-diffusion simulator and included nonradiative recombination. ${ }^{24}$ Other studies have considered transitions between multiple interstates. ${ }^{25,26}$

By means of an illustrative example, we present the results of Yoshida et al. ${ }^{22}$ from a self-consistent drift-diffusion simulation. Here, the optical generation $\left(G_{i j}\right)$ and recombination $\left(R_{i j}\right)$ rates between $\mathrm{IB}-\mathrm{VB}$ and $\mathrm{CB}-\mathrm{IB}$ are assumed to be locally balanced. Thus,

$$
G_{\mathrm{IC}}-R_{\mathrm{IC}}=G_{\mathrm{VI}}-R_{\mathrm{VI}}
$$

It should be noted that $G_{\mathrm{IC}}$ and $G_{\mathrm{VI}}$ are roughly proportional to $f_{\mathrm{I}}$ and $1-f_{\mathrm{I}}$, respectively, where $f_{\mathrm{I}}$ is the IB occupancy. Thus, the IB occupancy largely affects the optical generation rate and the potential profile. Energy gaps of CB-VB and IB-VB are 1.43 and $0.95 \mathrm{eV}$, and photon fluxes which can be absorbed by CB-IB and IB-VB transitions are equal, the effect of non-equal transition rates is discussed further in Sec. II D. The charge density is given by

$$
\rho=q\left(p-n_{\mathrm{C}}-n_{\mathrm{I}}+N_{D}^{+}-N_{A}^{-}\right)
$$

where $p, n_{\mathrm{C}}$, and $n_{\mathrm{I}}$ are hole, $\mathrm{CB}$, and IB electron concentrations. $N_{D}^{+}$and $N_{A}^{-}$are ionized donor and accepter concentrations. The device structure considered is a $p$-type top emitter, IB region, and $n$-type bottom base.

\section{Simulating the IBSC potential profiles}

The calculated band profiles of IBSC with non-doped IB are shown in Fig. 8 for the three different conditions of sun illumination; (a) no illumination (equilibrium), (b) 1 sun illumination, and (c) 1000 suns illumination, respectively. The potential profile under equilibrium (Fig. 8(a)) is very similar to that found in $p-i-n$ diodes except the region near the bottom base layer, where the quasi-Fermi level crosses the IB, indicating substantial filling of the band in this region. Hence, the large accumulation of electrons in the bottom base layer results in an acute drop in potential. Under solar illumination, the potential profiles greatly change, as shown in Figs. 8(b) and 8(c). The electron density in the IB increases through optical absorption and the potential profile resembles that of a $p-p^{-}-n$ diode. Increasing the solar concentration, the states in IB become significantly occupied and the quasi-Fermi levels of IB nearly coincide with $E_{\mathrm{I}}$, as seen in Fig. 8(c).

The band profiles of the IBSC with doped IB are shown in Fig. 9 for the three different conditions of solar illumination the same as in Fig. 8. The doping density is one half of the effective density of states in the IB. Because of the 
(a)

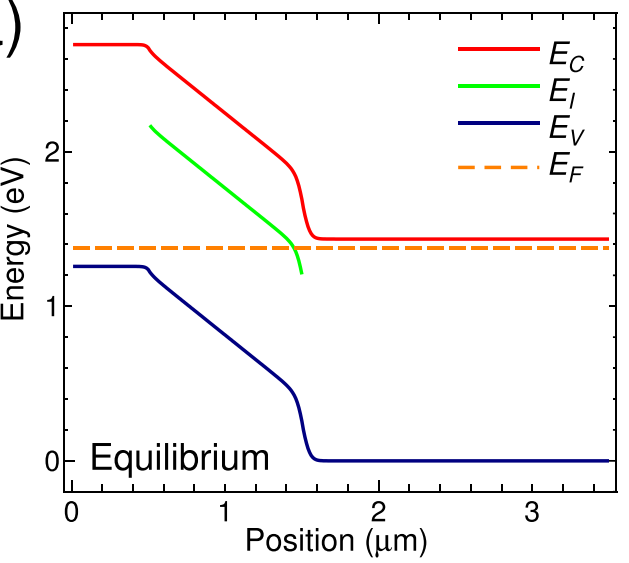

(b)

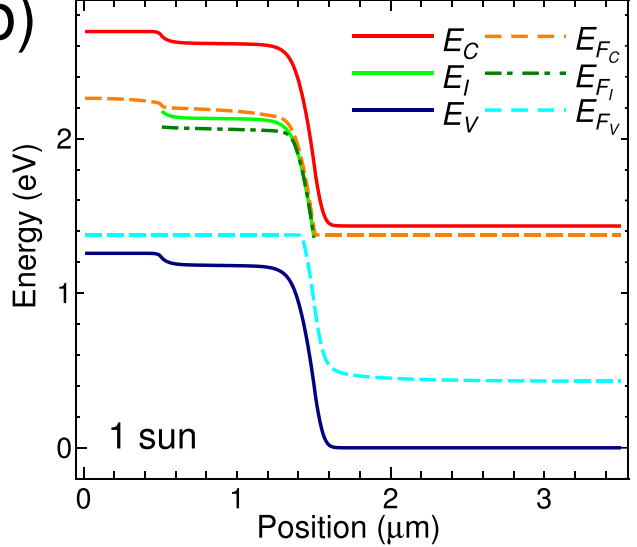

(c)

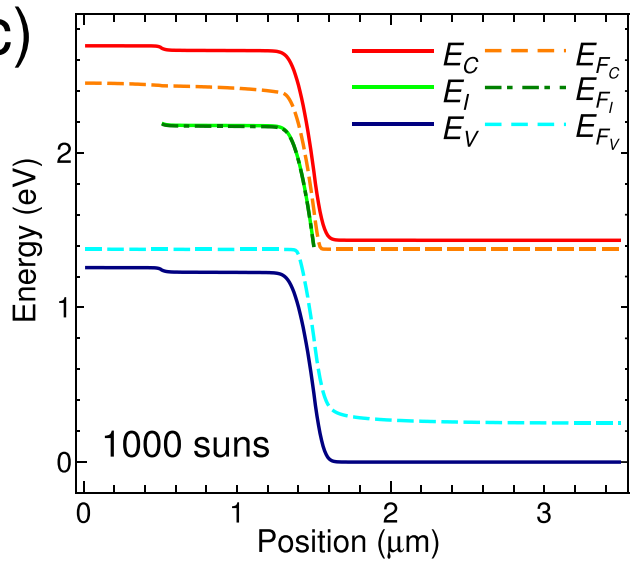

FIG. 8. Band profile of IBSC in short-circuit condition along $x$-direction with non-doped IB under (a) no illumination (equilibrium), (b) 1 sun illumination, and (c) 1000 suns illumination. $E_{\mathrm{C}}$ and $E_{\mathrm{V}}$ represent the band edge of $\mathrm{CB}$ and $\mathrm{VB}$, respectively, and $E_{\mathrm{I}}$ is that of IB. $E_{F_{\mathrm{C}}}, E_{F_{\mathrm{I}}}$, and $E_{F_{\mathrm{V}}}$ are the quasi-Fermi levels of electrons in $\mathrm{CB}$, IB and of holes in $\mathrm{VB}$, respectively.

ionized impurity in the IB region, the potential profile under equilibrium (no illumination) becomes similar to that in $p-n^{-}-n$ diodes (see Fig. 9(a)). The depletion regions are located only near the emitter and base layers. Figures 9(b) and $9(\mathrm{c})$ show that sun illumination drastically changes the potential profiles inside the cell. Compared with Fig. 9(a), the occupation probability near the edges of the IB region greatly changes and the depletion region spreads over the whole IB region. Consequently, the potential profile becomes similar to that in $p-i-n$ diodes. The quasi-Fermi levels of IB coincide with $E_{\mathrm{I}}$ over the whole region under 1000 suns and, thus, the energy difference of $E_{\mathrm{I}}$ and $E_{F_{\mathrm{I}}}$ becomes
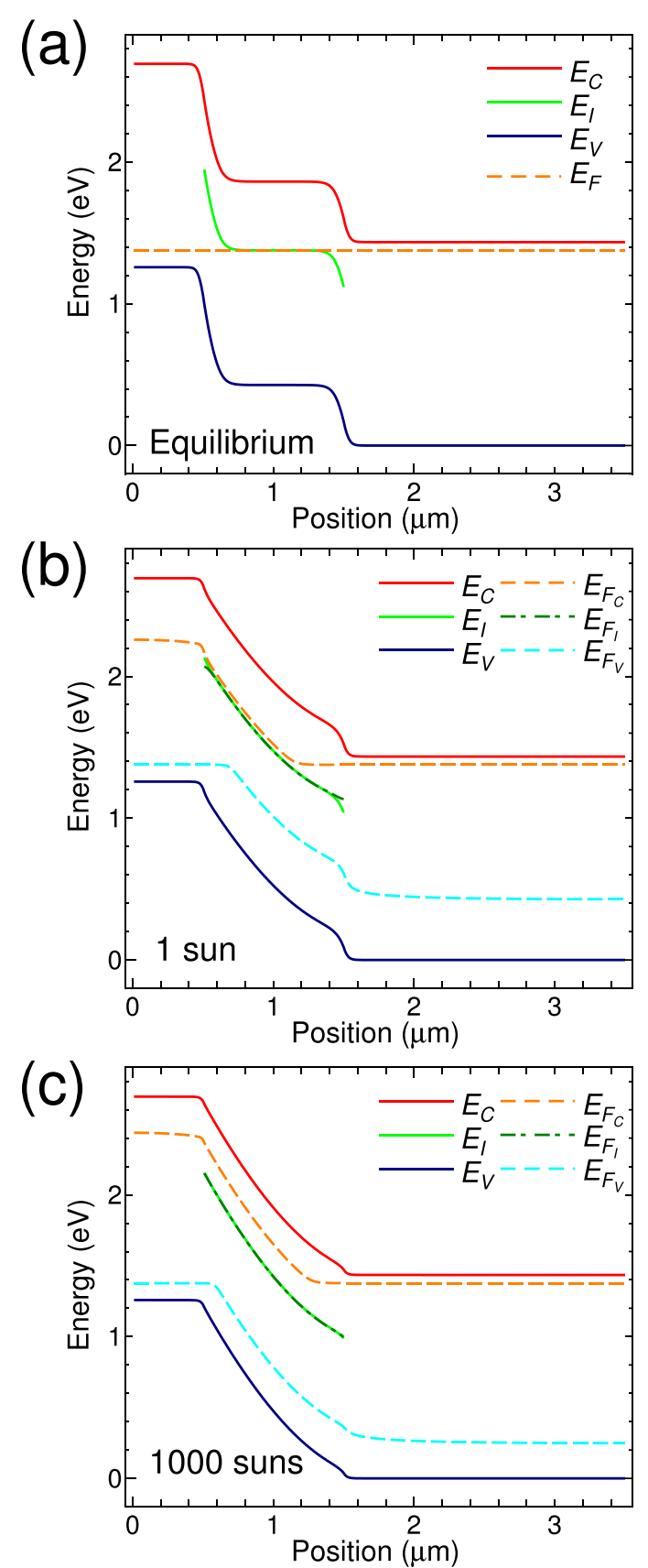

FIG. 9. Band profile of IBSC in short-circuit condition along $x$-direction with doped IB under (a) no illumination (equilibrium), (b) 1 sun illumination, and (c) 1000 suns illumination.

independent of doping density in IB. This indicates that the occupation probability in IB is determined by the photon density, which is often called as "photofilling." 27

In short, the presence of significant charge in the IB results in a sharp departure from the usual "depletion" approximation that is applied in a typical Shockley diode analysis of a semiconductor solar cell. The potential profile can only be determined through self-consistent simulation of the Poisson and drift-diffusion transport equations.

\section{Simulating IBSC solar cell performance}

The short-circuit current density $\left(J_{\mathrm{SC}}\right)$, open-circuit voltage $\left(V_{\mathrm{OC}}\right)$, and conversion efficiency as a function of light 
concentration factor $X$ for IBSCs and a bulk single-junction (GaAs like) control cell are shown in Fig. 10. As discussed earlier, $J_{\mathrm{SC}}$ for IBSC with a non-doped IB shows a strong dependence of $X$, whereas those for an IBSC with doped IB and a bulk single-junction control cell are nearly independent of $X$. However, as $X$ increases to $1000, J_{\mathrm{SC}}$ for the IBSC with a non-doped IB approaches that for IBSC with doped IB due to the photofilling effects. Figure 10(b) shows that the $V_{\mathrm{OC}}$ of IBSC with either non-doped or doped IB region is strongly dependent on $X$, while that for GaAs control cell is logarithmically dependent which is typical for a singlejunction solar cell. ${ }^{28}$ This is because that IBSC has a recombination process through IB in addition to $\mathrm{CB}-\mathrm{VB}$ recombination and this recombination mainly determines the $V_{\mathrm{OC}}$. In addition, the recombination process through IB is similar to Shockley-Read-Hall (SRH) recombination, and thus, IBSCs show stronger dependence on the concentration $X$ than the control cell. Under high concentration $(X>100)$, optical generation rates of $\mathrm{CB}-\mathrm{IB}$ and $\mathrm{IB}-\mathrm{VB}$ become enough large to suppress the recombination via IB and the $V_{\text {OC }}$ of IBSCs yield a value similar to that of the control cell.

Figure 10(c) shows that the conversion efficiency of all cells (IBSCs and control cell) increases as $X$ becomes larger. In particular, the light concentration dependence of conversion efficiency for IBSC with doped IB and GaAs control cell is determined by the $X$-dependence of $V_{\mathrm{OC}}$ because $J_{\mathrm{SC}}$ is nearly independent of $X$. However, under weak illumination, there is degradation of $V_{\mathrm{OC}}$ and fill factor $(F F)$ leading to a loss in efficiency. For the non-doped IB, there is also a strong reduction in $J_{\mathrm{SC}}$ further illustrating the need to photofill the IB when it is non-doped and the cell operated under anything other than highly concentrated light.

In the simulation above, the doping concentration is set to the optimal value of half the effective density of states of IB. This is because incident photon fluxes absorbed by CBIB and IB-VB transitions are almost the same, and thus, the optimal IB occupancy is almost 0.5. Therefore, the optimal doping concentration largely depends on the ratio of the two photon fluxes. However, the other doping concentrations in the top emitter and bottom base regions and the potential profile under the operation should also be considered. Thus, the simulation with including the whole cell structure is important especially under low light concentration.

\section{Interband recombination rates}

The analysis above considers the problem of sequential photon absorption via the IB, but assumed radiatively limited recombination. We now turn to an equally important aspect that of the interband recombination rate. For the IB to add to the solar cell efficiency, there must be a net generation of carriers via the IB. However, if the recombination rate is very fast compared to generation rate, then this will limit the carrier density that can develop in the IB.

The spontaneous emission rate, $r_{s p}$, is proportional to the density of photon states $\left(g_{\gamma}\right)$ and an integration over all possible carriers that can participate in a optical transitions while conserving energy and momentum

$$
r_{s p}(\hbar \omega)=g_{\gamma}(\hbar \omega) \int_{0}^{\infty} M(\epsilon, \hbar \omega) n_{u}(\epsilon) n_{o}(\epsilon+\hbar \omega) d \epsilon
$$

The condition for energy and momentum was derived in Sec. II A, therefore the spontaneous emission rate can be written as

$$
r_{s p}(\hbar \omega)=g_{\gamma}(\hbar \omega) M\left(\epsilon^{\prime}, \hbar \omega\right) n_{u}\left(\epsilon^{\prime}\right) n_{o}\left(\epsilon^{\prime}+\hbar \omega\right) .
$$

The total transition rate is found by integrating over all possible transition energies

$$
R_{s p}=\int_{0}^{\infty} r_{s p}(\hbar \omega) d(\hbar \omega) .
$$

A convenient and commonly used expression for the net integrated radiative recombination rate is given by the bimolecular recombination equation

$$
R_{s p}=B n p
$$

where $B$ is related to the absorption coefficient by ${ }^{29}$

$$
B=\frac{8 \pi}{h^{3} c^{2}} \int_{E g}^{\infty} \alpha(E) E^{2} \exp \left(\frac{-E}{k_{B} T}\right) d E .
$$

Equation (19) is valid for dilute carrier concentrations, as it begins to overestimate the recombination rate for degenerate carrier concentrations ( $\sim 10^{20} \mathrm{~cm}^{-3}$ in most III-V materials). There are two main factors that influence the interband radiative recombination:

(1) Equilibrium carrier density (doping).

(2) Absorption coefficient.

\section{Radiative lifetime and doping}

To consider the effect of carrier density in more detail, an illuminated semiconductor with excess number of electrons $\Delta n$, and holes $\Delta p$, generated by the absorption of light and equilibrium carrier concentrations $n_{0}$ and $p_{0}$ due to the intrinsic or extrinsic nature of the material are introduced. Therefore, the radiative recombination rate in Eq. (19) can be written as

$$
R_{s p}=B\left(n_{0}+\Delta n\right)\left(p_{0}+\Delta p\right)
$$

In the low-injection limit, where $\Delta n \ll\left(n_{0}+p_{0}\right)$, this leads to the rate equation for excess carrier concentration

$$
\frac{d}{d t} \Delta n(t)=-B\left(n_{0}+p_{0}\right) \Delta n(t)
$$

which yields a lifetime

$$
\tau_{\text {rad }}=\frac{1}{B\left(n_{0}+p_{0}\right)} .
$$

The main result is that the radiative lifetime, in the nondegenerate limit, is only dependent on the equilibrium carrier concentrations. For this reason, doping of semiconductor materials strongly influences the radiative lifetime. ${ }^{30}$ For example, 

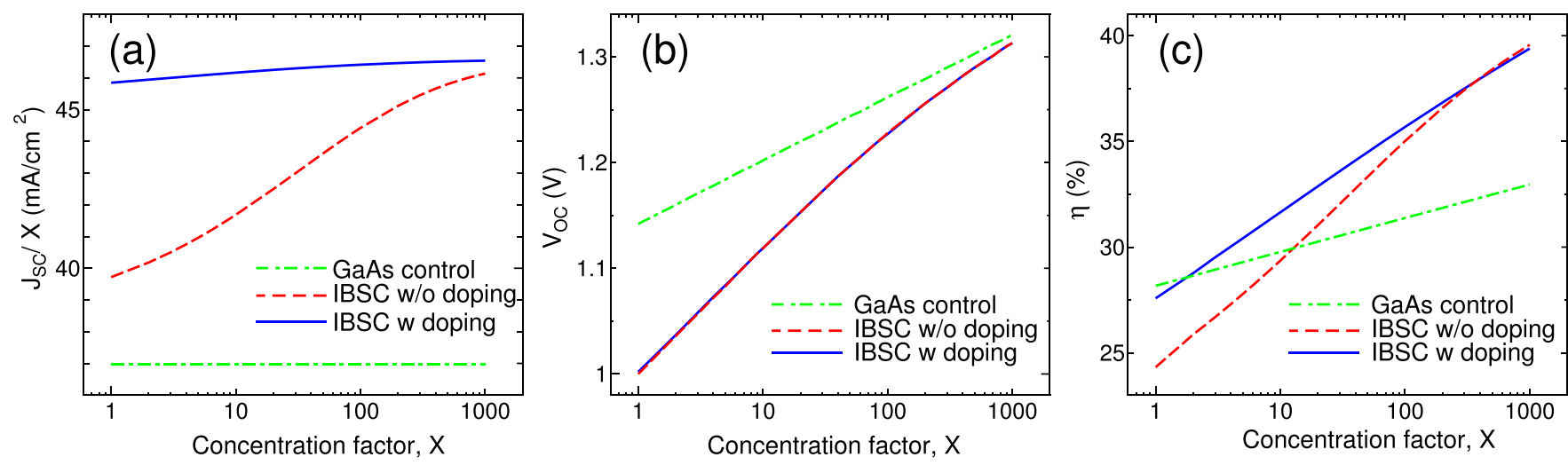

FIG. 10. (a) $J_{\mathrm{SC}}$, (b) $V_{\mathrm{OC}}$, and (c) conversion efficiency for IBSCs with non-doped and doped IB and for GaAs control cell as a function of the light concentration factor $X . J_{\mathrm{SC}}$ is normalized by the light concentration factor $X$. Reprinted with permission from Yoshida et al., J. Appl. Phys. 112, 084510 (2012). Copyright 2012 AIP Publishing LLC.

- Intrinsic semiconductors: The radiative lifetime for intrinsic GaAs is $\tau_{\text {rad }}=\left(B 2 n_{i}\right)^{-1}=2500 \mathrm{~s}$ using $B=10^{-10} \mathrm{~cm}^{3}$ $\mathrm{s}^{-1}$ and $n_{i}=2 \times 10^{6} \mathrm{~cm}^{-3}$. This is a remarkably long lifetime but arises simply from the low carrier density in intrinsic GaAs and hence a low probability for interaction.

- Extrinsic semiconductors: Doping a semiconductor such that one carrier type is in extreme abundance, significantly reduces the radiative lifetime. For $n$-type extrinsic GaAs, the radiative lifetime is $\tau_{\text {rad }}=\left(B N_{D}\right)^{-1}=1 \mathrm{~ns}$, with the donor concentration $N_{D}=10^{18} \mathrm{~cm}^{-3}$. In extrinsic materials, radiative recombination is very fast because an excess carrier (in this case the hole) will always in close proximity to electron and can therefore recombine rapidly.

\section{Radiative lifetime and absorption coefficient}

Absorption coefficient is the second major factor influencing the radiative recombination rate. A material with a high absorption coefficient will also, in general, have fast radiative recombination. To understand this better, the principle of detailed balance to radiative transitions has to be applied first

$$
r_{a}(\hbar \omega)-r_{s t}(\hbar \omega)-r_{s p}(\hbar \omega)=0 .
$$

Previously, the term $r_{a}(\hbar \omega)-r_{s t}(\hbar \omega)=W_{\mathrm{VC}}(\hbar \omega)$ was defined; therefore, the detailed balance condition can be written in terms of the absorption coefficient

$$
\alpha_{0}(\hbar \omega) I(\hbar \omega)\left(f_{\mathrm{V}}\left(\epsilon^{\prime}\right)-f_{\mathrm{C}}\left(\epsilon^{\prime}+\hbar \omega\right)\right)-r_{s p}(\hbar \omega)=0 .
$$

This implies that if $\alpha_{0}(\hbar \omega)$ increases it must be countered by a related increase in the spontaneous recombination rate. This can be stated succinctly as

$$
\alpha_{0}(\hbar \omega) \propto r_{s p}(\hbar \omega) \propto \frac{1}{\tau_{r a d}} .
$$

\section{Factors limiting the efficiency of IBSC}

Here, we draw on the elements from the previous discussion regarding absorption coefficient and radiative lifetime with a view to elucidate further how these processes affect the efficiency of an IBSC. We restrict our attention to the case of an InAs/GaAs quantum dot (QD)-IBSCs discussed experimentally more detail in Sec. IIID4. In our simulation, we have carefully treated the energy-band structure with the one-dimensional Poisson equation and the continuity equations, ${ }^{31}$ similar to the approach described in Sec. II B 1.

The key process of an IBSC comprises a two-step photon absorption (TSPA): absorption of a photon which promotes an electron from VB to IB and the subsequent absorption of a second photon which promotes an electron from the IB to $\mathrm{CB}$. All the charges in the IB are provided by optical transitions, either by the direct absorption of sub-bandgap photons or by recombination processes from higher laying bands. In steady-state conditions, the net absorptive (upwards) transition rates (VB to IB and IB to $\mathrm{CB}$, respectively) have to be balanced at each position in the device. This leads the balance equation (see also Eq. (14))

$$
G_{\mathrm{VI}}(x)-R_{\mathrm{VI}}(x)=G_{\mathrm{IC}}(x)-R_{\mathrm{IC}}(x) .
$$

Because QDs generate isolated states in the bandgap of the host material, a finite energetic bandwidth for the IB is assumed rather than a continuous band which extends to infinity. This assumption is consistent with a band with high effective mass (thus low curvature in $k$-space) and therefore also low electron mobility. Upon incorporating the effects of sub-bandgap absorption coefficients, the generationrecombination rates are represented by ${ }^{19}$

$$
\begin{array}{r}
G_{i j}=\iint \alpha_{i j} I(\hbar \omega) \exp \left(-\alpha_{i j} x\right) d(\hbar \omega) d x, \\
R_{i j}=\frac{8 \pi}{h^{3} c^{2}} \iint \frac{\alpha_{i j}(\hbar \omega)^{2}}{\exp \left(\frac{\hbar \omega-\Delta \mu}{k_{B} T}\right)-1} d(\hbar \omega) d x,
\end{array}
$$

where $\alpha_{i j}$ is the effective absorption coefficient, $I(\hbar \omega)$ is the photon flux calculated using the Planck formula, and $\Delta \mu_{i j}$ is the quasi-Fermi level splits. Here, a $6000 \mathrm{~K}$ blackbody is used to simulate the solar spectrum at maximum concentration. 


\section{Effect of carrier occupancy}

It is important to note that the sub-bandgap absorptions via IB depend on the probability of IB occupation, as discussed previously this is represented by

$$
\begin{aligned}
& \alpha_{\mathrm{IC}}=\alpha_{\mathrm{IC}, 0}\left(f_{\mathrm{I}}-f_{\mathrm{C}}\right), \\
& \alpha_{\mathrm{VI}}=\alpha_{\mathrm{VI}, 0}\left(f_{\mathrm{V}}-f_{\mathrm{I}}\right) .
\end{aligned}
$$

Assuming that the VB is completely full $\left(f_{\mathrm{V}}=1\right)$ and $\mathrm{CB}$ is completely empty $\left(f_{\mathrm{C}}=0\right)$ the equation can be simplified to $^{21,32}$

$$
\begin{gathered}
\alpha_{\mathrm{IC}}=f_{\mathrm{I}} \alpha_{\mathrm{IC}}^{100}, \\
\alpha_{\mathrm{VI}}=\left(1-f_{\mathrm{I}}\right) \alpha_{\mathrm{VI}}^{0},
\end{gathered}
$$

where the absorption coefficients are parameterised using $\alpha_{\mathrm{IC}}^{100}$ and $\alpha_{\mathrm{VI}}^{0}$ represent the maximum absorption coefficients for the IB to CB transition when the IB is completely full, and maximum absorption coefficient for the VB to IB transition when the IB completely empty, respectively. The absorption coefficient is therefore proportional to the FermiDirac distribution.

Combining Eq. (27) with the one-dimensional Poisson equation and the continuity equations, the IB occupation provability, energy-diagram, and conversion efficiency can be calculated. Here, it is assumed that the collection efficiency of photogenerated carriers to be unity. The absorption coefficients ( $\alpha_{\mathrm{IC}}^{100}$ and $\alpha_{\mathrm{VI}}^{0}$ ) are determined from the oscillator strength and the density of states but are independent of the probability of IB occupation. Equations (27) and (29) reveal that the photo-carrier distribution depends on $f_{\mathrm{I}}$, which is determined by the separate IB quasi-Fermi level. Under solar illumination, the QD number density is not sufficiently high to pin the quasi-Fermi level at the IB level because the total optical generation remains small. It should be noted that the IB quasi-Fermi level is approximately parallel to the IB level, and $f_{\mathrm{I}}$ depends to a small extent on the spatial position because the light intensity decays with the penetration depth. The net generation rates clearly reveal this decay process. Because effective absorption coefficients depend on $f_{\mathrm{I}}$, they do not follow a simple exponential distribution. Besides, the net generation rate curves of the VB-IB and IB-CB transitions almost overlap each other, indicating that the numerical resolutions are accurate and stable.

Figure 11(a) shows the efficiency contour plots calculated for $500 \mathrm{~nm}$ InAs/GaAs QD-IBSCs under maximum concentration. These results reveal that there is no defined value for optimised $E_{\mathrm{I}}$ in any material system; the optimised $E_{\mathrm{I}}$ depends on the ratio of absorption coefficients, $\alpha_{\mathrm{IC}}^{100} / \alpha_{\mathrm{VI}}^{0}$. When $\alpha_{\mathrm{IC}}^{100} / \alpha_{\mathrm{VI}}^{0}$ is decreased, increasing the IB levels increases the photon flux from IB to $\mathrm{CB}$ and decreases the photon flux from VB to IB, eventually compensating for the decrease in $\alpha_{\mathrm{IC}}^{100} / \alpha_{\mathrm{VI}}^{0}$. Similarly, when $\alpha_{\mathrm{IC}}^{100} / \alpha_{\mathrm{VI}}^{0}$ is increased, optimum IB will shift in the opposite direction. The maximum efficiency is $52.8 \%$ when $\alpha_{\mathrm{IC}}^{100} / \alpha_{\mathrm{VI}}^{0}$ is 0.2 and $E_{\mathrm{I}}$ is $1.0 \mathrm{eV}$. Overall, a large $\alpha_{\mathrm{IC}}^{100}$ is necessary for high conversion efficiency. In addition, increasing total thickness of the QD layer can partially address the demand for a large $\alpha_{\mathrm{IC}}^{100}$.
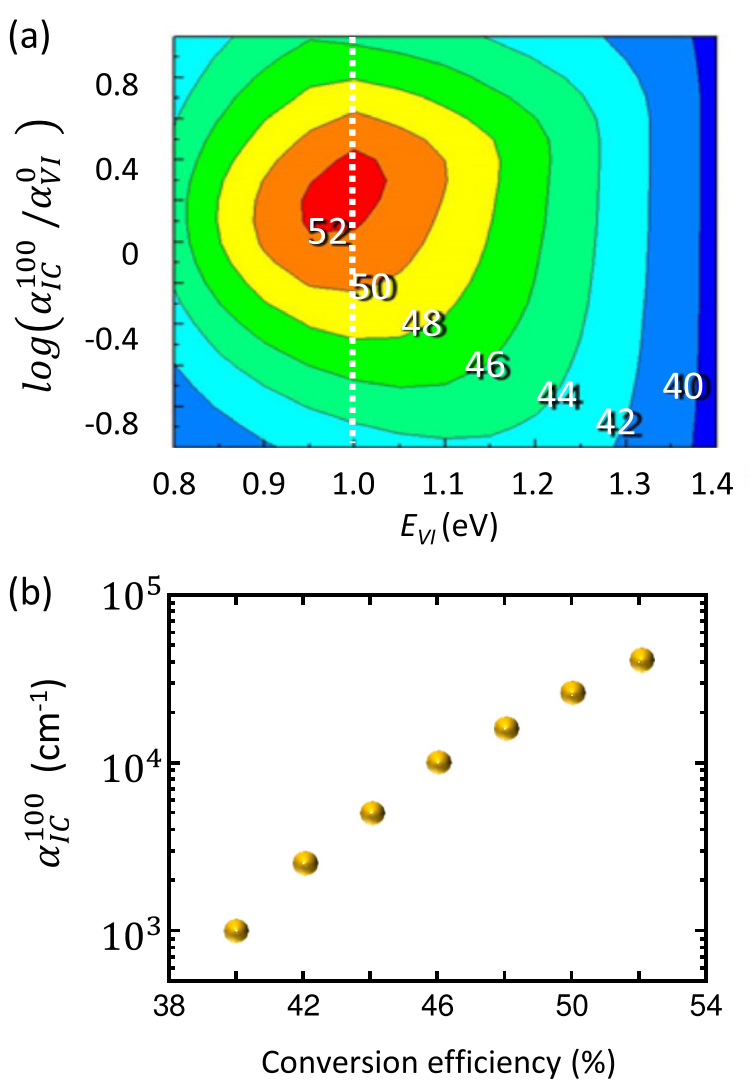

FIG. 11. (a) Efficiency counter plot in $500 \mathrm{~nm}$ InAs/GaAs QD-IBSCs under maximum concentration. (b) A relation between inter-subband absorption coefficient and the conversion efficiency.

Figure 11(b) shows a relation between inter-subband absorption coefficient and the conversation efficiency, evaluated from this result for InAs/GaAs QD-IBSCs. The intersubband absorption coefficient greater than $1000 \mathrm{~cm}^{-1}$ is demanded to surpass single-junction limit. Furthermore, $50 \%$ efficiency requires a coefficient greater than $10000 \mathrm{~cm}^{-1}$, which is close in magnitude to that of the interband transition. These results are supported by the simpler yet more fundamental thermodynamic models of the IBSC which reveal high efficiency is achieved when all optical transitions occurs at similar rates.

\section{Influence of electron lifetime}

Next, the effects of electron lifetime in the IB on the conversion efficiency of QD-IBSCs are considered. A wellknown detailed balance calculation of the high conversion efficiency for an ideal IBSC is neglecting nonradiative lifetime. However, generally, QDs have a finite nonradiative lifetime and may participate in optical transitions because they act as recombination centers. Figure 12 shows conversion efficiency calculated by using the detailed balance theory as a function of the nonradiative electron lifetime in the IB. Here, we assumed 10 QD layers with an in-plane QD density of $10^{10} \mathrm{~cm}^{-2}$. The conversion efficiency depends strongly on the electron lifetime. Electron lifetime in QDs is influenced by carrier recombination strength, thermal escape, and tunneling escape. A long electron lifetime will be accomplished by controlling the recombination rate, ${ }^{12,33}$ using a high 


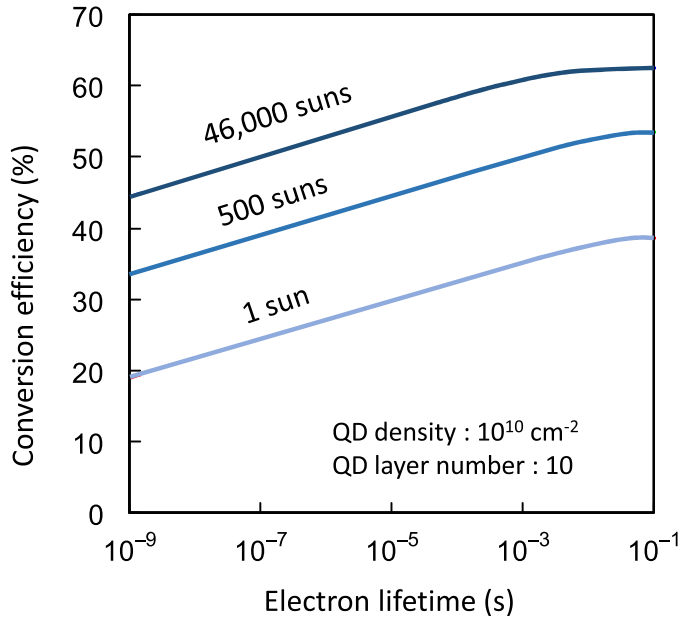

FIG. 12. Conversion efficiency calculated by using the detailed balance theory as a function of electron lifetime in the IB.

potential barrier preventing thermal carrier escape from QDs, ${ }^{34-36}$ and introducing an electric field damping layer. ${ }^{37}$

Introducing a RB into the solar cell, as shown in Fig. 4, assists with the problem of maintaining long carrier lifetime. Assuming that the VB-IB transition is direct and allowed, a typical electron lifetime of the order of $10 \mathrm{~ns}$ can be assumed. Assuming Boltzmann statistics for the electron distribution, introducing the relaxation step of $\Delta E$ will increase the lifetime by a factor $\exp \left(\Delta E / k_{B} T\right)$. This is responsible for intermediate state lifetimes of order $100 \mu$ s measured in molecular up-conversion systems. ${ }^{38,39}$ Further, the introduction of the fourth, RB also brings with it an advantage in terms of occupancy of the levels. The position of the IB Fermi energy ensures that the IB is relatively empty, thereby ensuring the VB-IB transition is strong, yet the RB is full, hence a strong RB-CB transition strength.

In this section, requirements for strong optical transitions in IB materials have been reviewed. The principle difficulty of using the IB approach for photovoltaics stems from the dual nature of the IB: it must have precisely the right carrier occupancy such that it can participate in absorptive optical transitions which promote electrons in-to and out-of the IB. If the occupancy in the IB is too low, the second optical transition (from the IB to CB) is strongly suppressed. Conversely, if the occupancy is too high, the first optical transitions (from the VB to IB) are strongly bleached. This constraint leads to severe current mismatch between the two junctions (and thus very low efficiency) when the IB is not half-filled. Current QD, quantum well (QW), dilute nitride, and dilute oxide approaches discussed in Sec. III suffer from very low occupancy in the IB so for this reason, IB operation is limited by the IB to $\mathrm{CB}$ transition.

\section{REVIEW OF PRESENT STATUS ON SOLAR CELL DEVELOPMENTS}

\section{A. Highly mismatched alloys}

\section{HMA materials and electronic properties}

Highly mismatched alloys (HMAs) are a class of semiconductors containing isoelectronic elements with very large differences in terms of atom size, ionicity, and electronegativity, for instance, $\mathrm{Ga}(\mathrm{N}, \mathrm{As})$ in which $\mathrm{N}$ ions substitute the As sites as well as $\mathrm{Zn}(\mathrm{Te}, \mathrm{O})$ alloys in which $\mathrm{O}$ ions substitute the Te sites. These alloys have very unusual electronic/ optical properties, for example, alloying of $\mathrm{Ga}(\mathrm{N}, \mathrm{As})$ can result in an unusual compositional behaviour of the bandgap. These HMAs have been investigated for optoelectronic applications on account of their strong bandgap reduction, notably the use of GaInNAs, in both edge emitting and vertical cavity surface emitting lasers, semiconductor optical amplifiers, photodetectors, and optical modulators. ${ }^{40}$ Considerable attention has also been drawn due to their potential for $1-\mathrm{eV}$ subcell in ultrahigh efficiency multijunction solar cells that are based on existing GaAs technologies. ${ }^{41}$ Recently, a triple-junction solar cell that contains a GaInNAsSb bottom cell presented a record efficiency solar cell performance of $43.5 \%{ }^{42}$ This section reviews recent reports that extend further the potential of the HMAs toward application into the developing field of thin-film IBSC.

The alloy bandgap varies as a function of composition and can be estimated to a first degree by the linear interpolation between the values of the endpoint materials, A and B, assumed by the virtual crystal approximation (VCA), where the potential of the periodic crystal is taken as an average of the atomic potentials of the constituents. ${ }^{43}$ However, semiconductor alloys usually exhibit some deviation, or bowing, away from this trend due primarily to constituent mismatch and disorder related potential fluctuations. Instead, the bowing is typically described by a quadratic relationship between the two gaps using an empirically determined nonlinear or bowing parameter $b$ to characterize the degree of divergence from the $\mathrm{VCA}^{43}$

$$
E_{g}^{\text {alloy }}(x)=x E_{g}^{A}+(1-x) E_{g}^{B}-b x(1-x) .
$$

Here, $x$ is the fraction of compound A mixed in compound B. Room temperature (RT) bandgaps are 3.4 and $1.4 \mathrm{eV}$, respectively, for the endpoint materials $\mathrm{GaN}$ and $\mathrm{GaAs}$ for the $\mathrm{GaN}_{x} \mathrm{As}_{1-x}$ HMA. Previous investigations have found a large bowing parameter $b=18 \mathrm{eV}$ for this ternary alloy. ${ }^{44}$ While the values of $b$ required to satisfy the bowing in most alloys are typically smaller than their bandgaps, such as in the direct bandgap case of $\mathrm{Al}_{x} \mathrm{Ga}_{1-x} \mathrm{As}(b=0.437),{ }^{45}$ the bowing parameter determined for $\mathrm{GaN}_{x} \mathrm{As}_{1-x}$ is more than ten times the gap of GaAs. Although the large bowing was expected to produce a semimetallic overlap at intermediate compositions, ${ }^{46}$ more detailed studies found a reduction of the bowing parameter with increasing composition. ${ }^{47-49}$ In addition to the bandgap bowing issue, further optical investigations of GaNAs have shown that at energies higher than the fundamental bandgap there is an additional transition denoted $E_{+} \cdot{ }^{50,51}$ These observations suggest that a single bowing parameter no longer adequately describe the trend of the bandgap, and more advanced theory is required. Similarly, large bandgap bowing observed in other III-V, II-VI, and IV-IV compounds including $\mathrm{GaN}_{x} \mathrm{As}_{1-x-y} \mathrm{P}_{y},{ }_{5}$ $\mathrm{GaBi}_{x} \mathrm{As}_{1-x},{ }^{53} \mathrm{ZnO}_{x} \mathrm{Te}_{1-x},{ }^{54} \mathrm{ZnTe}_{x} \mathrm{~S}_{1-x},{ }^{55}$ and $\mathrm{Sn}_{x} \mathrm{Ge}_{1-x} .{ }^{56}$

This anomalous behaviour has been understood in terms of a band anticrossing (BAC) model..$^{51,57,58}$ This model has 
also been invoked to explain Mn-derived impurity band in a dilute magnetic alloy $(\mathrm{Ga}, \mathrm{Mn}) \mathrm{As} .{ }^{59}$ A highly electronegative, isoelectronic impurity atom, such as $\mathrm{N}$ in GaAs or $\mathrm{O}$ in $\mathrm{ZnTe}$, introduces localized $s$-like states near the CB edge of the host compound, while a large metallic impurity atom with a much lower ionization energy than that of the host anion, i.e., $\mathrm{As}$ in $\mathrm{GaN}$ or $\mathrm{Te}$ in $\mathrm{ZnO}$, introduces localized $p$-like states near the VB edge. The BAC model predicts that an anticrossing interaction between the localized states of these impurity atoms and the extended states of the host leads to a restructuring of the $\mathrm{CB}$ or the VB into $E_{+}$and $E_{-}$ subbands. Wu et al. ${ }^{60}$ have derived the BAC model using the Green's functions of Anderson many impurity model ${ }^{61}$ in the coherent potential approximation, and the dispersion relation for the impurity composition $x$ are then determined from the two-state like eigenvalue problem

$$
\left|\begin{array}{cc}
E_{k}^{c}-E(k) & C_{\mathrm{A}} \sqrt{x} \\
C_{\mathrm{A}} \sqrt{x} & E_{\mathrm{D}}+i \Gamma-E(k)
\end{array}\right|
$$

The term $\Gamma$ is the energy broadening of impurity level $E_{\mathrm{D}}, E_{k}^{c}$ is the unperturbed $\mathrm{CB}$ dispersion, $C_{\mathrm{A}}$ is the coupling parameter interpreting as hybridization strength of two anion elements. In the event that $\Gamma=0$, the dispersion relation is given as ${ }^{62}$

$$
E_{ \pm}(k)=\frac{1}{2}\left[\left(E_{k}^{c}+E_{\mathrm{D}}\right) \pm \sqrt{\left(E_{k}^{c}+E_{\mathrm{D}}\right)^{2}+4 C_{\mathrm{A}}^{2} x}\right] .
$$

The dispersion of two $\mathrm{CB}$ subbands $E_{-}$and $E_{+}$can be observed in the schematic examples of calculated band structures in Fig. 13 based on the BAC model. ${ }^{63}$ The effect of the interaction is most pronounced for the states located close to impurity state $E_{\mathrm{D}}$. When $E_{\mathrm{D}}$ is located within the $\mathrm{CB}$ of the matrix (for example, $\mathrm{N}$ state lies at about $0.25 \mathrm{eV}$ above the $\mathrm{CB}$ in GaAs (Ref. 51)), as depicted in Fig. 13(a), the CB
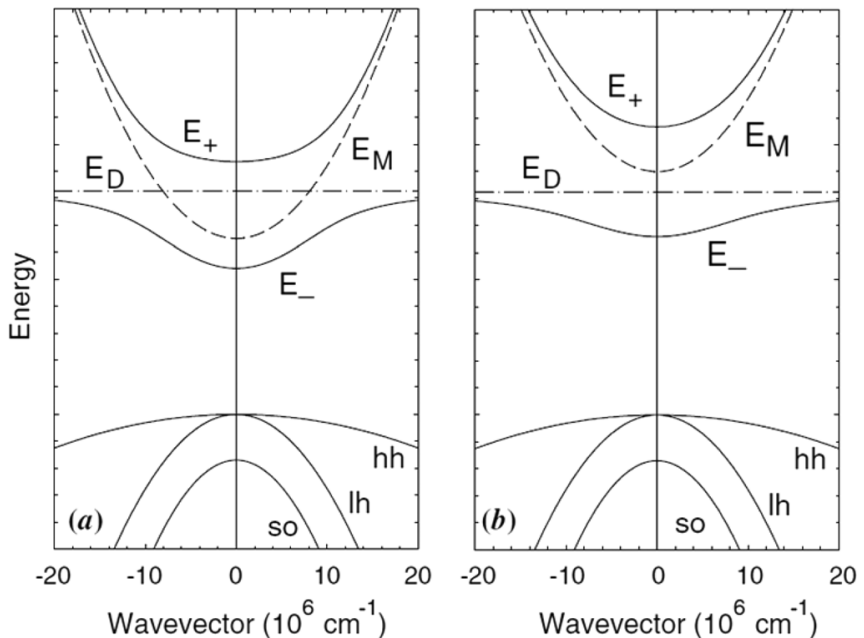

FIG. 13. Illustration of the effects of BAC on the $\mathrm{CB}$ in the vicinity of the $\Gamma$-point minimum for two cases: (a) The isoelectronic $\mathrm{N}$ induced localized state is resonant with the $\mathrm{CB}$ and (b) the localized state is located below the $\mathrm{CB}$. The solid curves are the restructured $E_{-}$and $E_{+}$subbands resulting from the BAC interaction between the localized states (dashed-dotted line) and the extended states of the $\mathrm{CB}$ (broken line). Reprinted with permission from Shan et al., J. Phys.: Condens. Matter. 16, S3355 (2004). Copyright 2004 Institute of Physics. states at the $E_{-}$edge retain mostly the extended $E_{\mathrm{M}}$-like character ( $E_{k}^{c}$ in Eqs. (35) and (36)) and those at the $E_{+}$edge have a more localized $E_{\mathrm{D}}$-like character. The $E_{-}$subband narrows drastically as the energy position of the $E_{\mathrm{D}}$ level moves down relative to the bottom of the CB. Narrowing of the band indicates a gradually increased contribution of the localized nature to the lowest subband, leading to a highly nonparabolic dispersion relationship that induces an enhancement of the effective mass and the density of states in the lower subband. When $E_{\mathrm{D}}$ is located below the CB edge (for example, $\mathrm{N}$ state lies at about $0.1 \mathrm{eV}$ below the $\mathrm{CB}$ in $\mathrm{GaP}$ (Ref. 51) or O state at about $0.4 \mathrm{eV}$ below $\mathrm{ZnTe}$ (Ref. 54)), as illustrated in Fig. 13(b), subbands $E_{-}$and $E_{+}$ switch their character: the $E_{-}$subband states assume a highly localized nature and the $E_{+}$subband states possess the character of the extended state.

Shown in Fig. 14 are photo-modulated reflectance (PR) spectra of metal-organic chemical vapor deposition (MOCVD) grown $\mathrm{GaN}_{x} \mathrm{As}_{1-x}$ thin films. ${ }^{51}$ In addition to the PR spectral features related to the transition from the top of the $\mathrm{VB}\left(E_{\mathrm{V}}\right)$ to $E_{-}$and the transition from the top of the split off VB to $E_{-}\left(E_{-}+\Delta_{0}\right.$ transition), an extra feature due to $E_{\mathrm{V}}$ to $E_{+}$transition appears at higher energies in the PR spectra. With increasing $\mathrm{N}$ concentration, the $E_{-}$and $E_{-}+\Delta_{0}$ transitions shift to lower energy and the $E_{+}$transition moves in the opposite direction toward higher energy. ${ }^{51}$ From the top of $E_{\mathrm{V}}$, the best fits to the data using the BAC model yield the energy of the nitrogen state, $E_{\mathrm{N}}=E_{\mathrm{V}}+1.65 \mathrm{eV}$ at atmospheric pressure, and the interaction parameter $C_{\mathrm{A}}=2.7 \mathrm{eV}$.

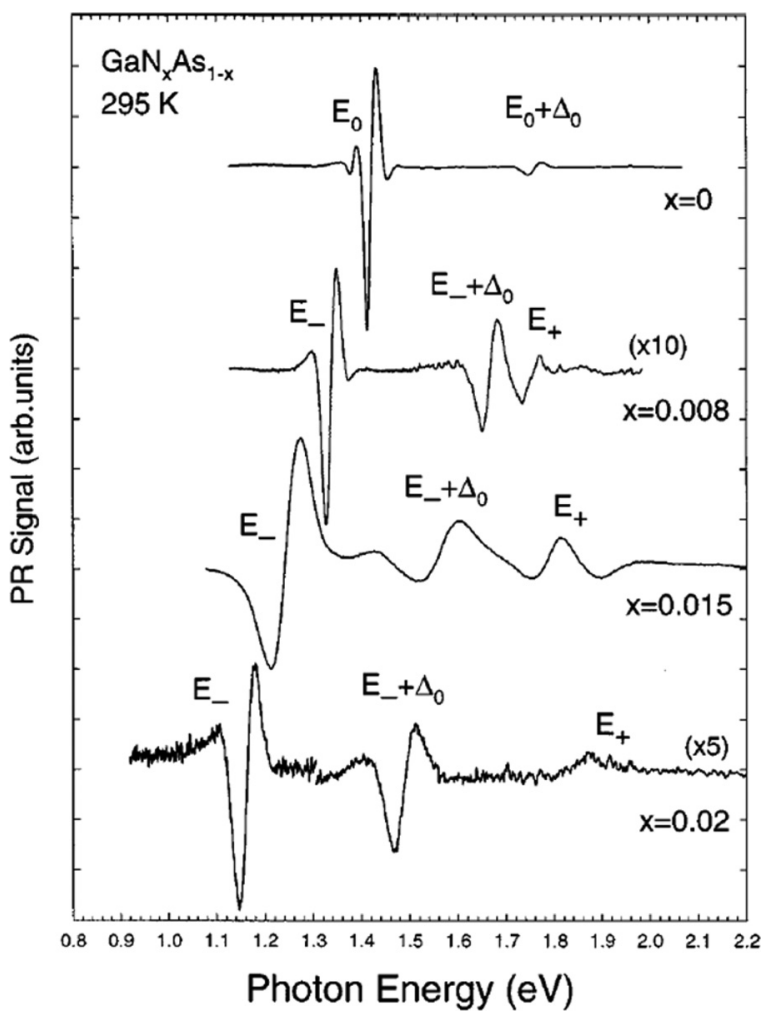

FIG. 14. PR spectra of $\mathrm{GaN}_{x} \mathrm{As}_{1-x}$ thin films with different $\mathrm{N}$ compositions. Reprinted with permission from Shan et al., J. Appl. Phys. 86, 2349 (1999). Copyright 1999 AIP Publishing LLC. 
In II-VI ZnTe system, addition of a small amount of isoelectronic Oxygen $(\mathrm{O})$ leads to the formation of a narrow, O-derived band $\left(E_{-}\right)$of extended states located well below the $\mathrm{CB}\left(E_{+}\right)$edge of the ZnTe. ${ }^{54,64}$ The key difference of this system from the III-V GaNAs system is that the IB is relatively localized in its band dispersion since the $\mathrm{O}$ level exists $0.4 \mathrm{eV}$ below the $\mathrm{CB}$ of $\mathrm{ZnTe}$. Figure 15 shows the transition energies from the VB to the $E_{-}$and $E_{+}$bands as a function of $\mathrm{O}$ composition $x$ of $\mathrm{ZnMnTe}_{1-x} \mathrm{O}_{x}$ layers synthesized using pulsed laser melting followed by rapid thermal annealing. With increasing $x$, the increasing difference between the $E_{-}$and $E_{+}$location shows a typical behavior for interacting two-level systems. The coupling parameter $C_{\mathrm{A}}$ in $\mathrm{ZnTe}_{1-x} \mathrm{O}_{x}$ is estimated to be $3.2 \mathrm{eV},{ }^{65}$ and this value can be compared with those of $\mathrm{ZnSe}_{x} \mathrm{Te}_{1-x}(1.0 \mathrm{eV}), \mathrm{ZnS}_{x} \mathrm{Te}_{1-x}$ $(1.0 \mathrm{eV}), \mathrm{ZnO}_{x} \mathrm{Se}_{1-x}(1.8 \mathrm{eV})$, and $\mathrm{GaN}_{x} \mathrm{As}_{1-x}(2.7 \mathrm{eV})$ systems. ${ }^{57,62,66}$ In the BAC model, the magnitude of this matrix element depends on the electronegativity difference between the matrix anion elements, ${ }^{57}$ and this difference is larger between $\mathrm{O}$ and $\mathrm{Te}(\Delta \chi=1.4 \mathrm{eV})$ than that between Se and $\mathrm{Te}(\Delta \chi=0.3), \mathrm{S}$ and $\mathrm{Te}(\Delta \chi=0.4)$, Se and $\mathrm{O}(\Delta \chi=1.1)$, and $\mathrm{N}$ and As $(\Delta \chi=1.0)$. This trend is in good agreement with obtained coupling parameter for $\mathrm{ZnTeO}$.

The key feature of a HMA is the formation of two subbands due to the highly mismatched impurity-induced splitting of the CB. This feature allows one choosing extra path for photon absorption (three paths in contrast to only one-path in $\mathrm{N}$-free GaAs host or O-free ZnTe). Further, using suitable amount of $\mathrm{N}$ or $\mathrm{O}$ composition, the multiple bandgaps can be optimized which add an additional advantage of the HMA system for IBSC application under desired level of solar concentration. Note that the theoretical background of the HMA system discussed so far has focused on the BAC

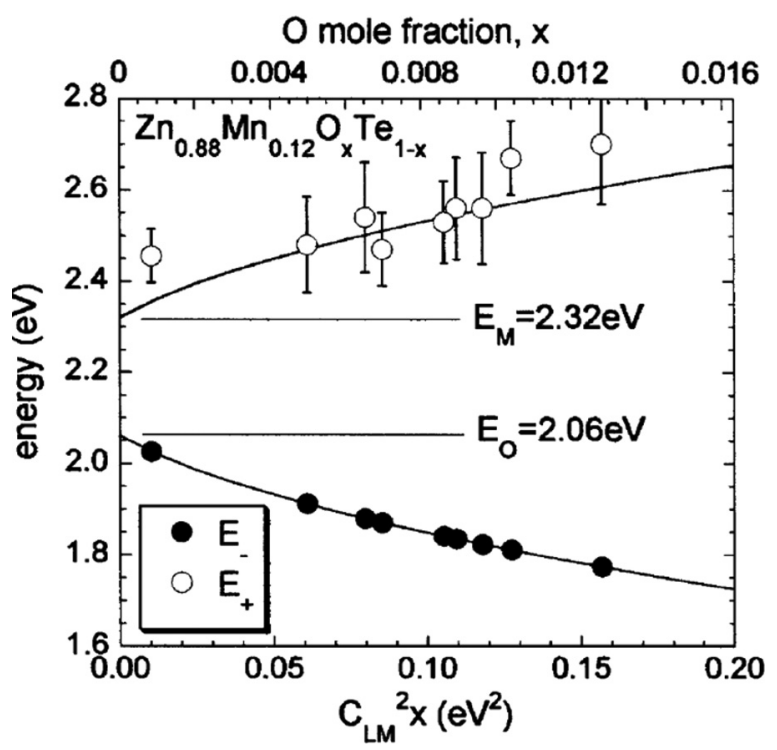

FIG. 15. Experimentally obtained energy positions of $E_{-}$(closed circles) and $E_{+}$(open circles) for the $\mathrm{Zn}_{0.88} \mathrm{Mn}_{0.12} \mathrm{O}_{x} \mathrm{Te}_{1-x}$ alloys plotted against the $\mathrm{O}$ mole fractions $x$. The values of $E_{-}$and $E_{+}$calculated according to the BAC model are plotted as solid lines. Here, the value of the coupling parameter $C_{\mathrm{LM}}$ (or $C_{\mathrm{A}}$ in Eq. (36)) has been assumed $3.5 \mathrm{eV}$ for the ZnMnTe host. Reprinted with permission from Yu et al., Phys. Rev. Lett. 91, 246403 (2003). Copyright 2003 American Physical Society. model despite a number of theoretical approaches have been applied that focus on the interaction of N-clusters. ${ }^{67-69}$ The simplicity and ease of interpretation of BAC model allows one designing multiple bands for certain impurity composition using an analytical expression. The experimental results obtained so far can be fairly explained by the BAC prediction. Yet some of the required feature for IBSC application needs more investigation, for example, nature of transition strength between the $E_{-}$and $E_{+}$subbands. Experimental data of GaNAs alloy using PR show that the PR magnitude of $\mathrm{VB}-E_{+}$transition is weak compared with the VB- $E_{-}$transition. $^{70}$ On the other hand, the direct measurement of $E_{-}-E_{+}$transition is not straightforward. The allowed dipole moments calculated for $E_{-}$and $E_{+}$inter-subbands in a GaNAs/AlGaAs QW using a ten-band $k \cdot p$ method were in the range of 2.16-3.13 $\AA^{71}$ These values, when considered as the upper limit for the GaNAs bulk, are fairly comparable to that of the bulk GaAs VB-CB transition $(5.81 \AA) .{ }^{72} \mathrm{In}$ experiments, as discussed in Secs. III A 2 and III A 3, an evidence of the photocurrent production aided by the $E_{-}-E_{+}$ absorption is presented. For bulk $\mathrm{ZnTeO}$, large oscillator strengths of the IB states to the VB and CB were calculated by Lee and Wang using density functional theory (DFT) ${ }^{73}$ Projection analyses show that the IB states mostly consist of $\mathrm{Zn} s$-states and $\mathrm{O} s$ - and $p$-states, ${ }^{73,74}$ and the strong oscillator strengths from IB to both VB and $\mathrm{CB}$ are ascribed to the mixed $s$ and $p$ characters of the IB states. ${ }^{73}$

However, HMAs are generally difficult to prepare due to large miscibility gaps. For example, in GaNAs system, one problem is the low equilibrium solubility of $\mathrm{N}$ in GaAs, which is only on the order of $10^{14}$ atoms $\mathrm{cm}^{-3} \cdot{ }^{75}$ Low growth temperatures below $600^{\circ} \mathrm{C}$ were used to kinetically limit phase separation, and crystals well above $1 \% \mathrm{~N}\left(10^{20}\right.$ atoms $\mathrm{cm}^{-3}$ ) in $\mathrm{GaAs}$ have been grown by metalorganic vapor phase epitaxy (MOVPE), molecular beam epitaxy (MBE), metalorganic MBE (MOMBE), and chemical beam epitaxy (CBE), etc. ${ }^{76,77}$ Under these conditions, kinetic effects at the growth surface likely play a dominant role, and motivated investigating role of surfactants, which are typically elements with small incorporation and desorption coefficients and accumulate on the surface during growth, thus changing growth properties. ${ }^{77-79}$ Also post growth annealing results in significant improvement of the optical qualities. ${ }^{80-82}$ Further, the observed rate of incorporation of $\mathrm{N}$ content in GaNAs, and correlation between $\mathrm{N}$ and In content in GaInNAs were found strongly dependent on the growth technique and precursor used. Other non-equilibrium preparation method such as ion implantation followed by shorttime annealing also made it possible to synthesize some other HMAs. ${ }^{54}$ As a new class of artificial materials, HMAs are still in the experimental stages of their development.

In this context, application of HMAs to IBSCs adds extra level of complication which encompasses full structure device design including substrate selection, diode formation with suitable level of doping, electrical isolation of IB, and a suitable window layer for a functional solar cell. The two IBSC systems of group III-V (GaNAs) and II-VI ( $\mathrm{ZnTeO})$ semiconductors have demonstrated potential for IBSC application. Sections III A2 and III A 3 review the 
characterization of the two systems composing full structures of IBSCs. Experimental data of photocurrent production using a TSPA setup are also introduced which is a key method for demonstrating the direct evidence of multi-band activity in a solar cell. More recent developments of these two systems are also discussed.

\section{III-V GaNAs-based thin-film IBSC}

Using the unique features of the multiple electronic band character of GaNAs alloys, López et al. have designed, fabricated, and tested an IBSC for the first time. ${ }^{83}$ The device demonstrates an optical activity of three energy bands that absorbs the crucial part of the solar spectrum, and converts into electrical current. Structures were prepared utilizing MOCVD.

Two device structures were designed with and without layers blocking charge transport in the IB. The device structures are shown in Figs. 16(a) and 16(b) together with the energy band diagram in which the energy band structure of the GaNAs layers was calculated using the BAC model. The structure with a blocked IB (BIB) is sandwiched between $\mathrm{AlGaAs}$ layers and has the blocking layers on both the surface and the substrate sides, whereas the structure with an unblocked IB (UIB) has a heavily doped InGaP layer on the substrate side, and the IB is connected to the substrate side of the device. The UIB device plays the role of a reference sample.

The EQE results of the above devices are shown in Fig. 17(a) and each contribution due to different transitions is assigned by the PR results in Fig. 17(b). In the UIB device, electrons excited to either the IB or the CB are collected at the substrate side, whereas the VB holes are driven to the surface contact. The IB plays the role of the CB and the device acts as a single bandgap solar cell whose characteristics are determined by the bandgap of about $1.1 \mathrm{eV}$ between the IB and the VB. On the other hand, in the BIB device the backside blocking layer prevents transfer of electrons from the IB to the back contact. $J-V$ characteristics for the two types of structures obtained under solar spectrum illumination with the intensity of about $20 \times$ air mass (AM) 1.5 are shown in Fig. 18. The UIB structure shows a $V_{\mathrm{OC}}$ of about $0.42 \mathrm{~V}$, whereas the BIB structure has a significantly higher $V_{\mathrm{OC}}$ of $0.92 \mathrm{~V}$. The small $V_{\mathrm{OC}}$ and large value of voltage offset $0.68 \mathrm{eV}$ from its bandgap $(1.1 \mathrm{eV})$ in the UIB structure is consistent with a relatively poor quality junction. However, the large, more than twofold increase of $V_{\mathrm{OC}}$ in the BIB structure can be associated with a gap much larger $1.1 \mathrm{eV}$ meaning that the largest bandgap of $2 \mathrm{eV}$ between $\mathrm{CB}$ and VB is responsible for the charge separation in this structure.

A probable cause for the high $V_{\mathrm{OC}}$ in the BIB device can be ascribed to the photo-generated carriers associated with the $E_{g}=1.42 \mathrm{eV}$ of the GaAs substrate, which is also a component of the structures. The spectral dependence of EQEs in Fig. 17(a) shows two thresholds in the BIB device compared to a single threshold at $1.1 \mathrm{eV}$ in the UIB. The thresholds at $1.1 \mathrm{eV}$ and $2.0 \mathrm{eV}$ correspond to the transitions from $\mathrm{VB}$ to IB and VB to CB in GaNAs, respectively. Although a contribution from GaAs to the EQE can be observed in the UIB
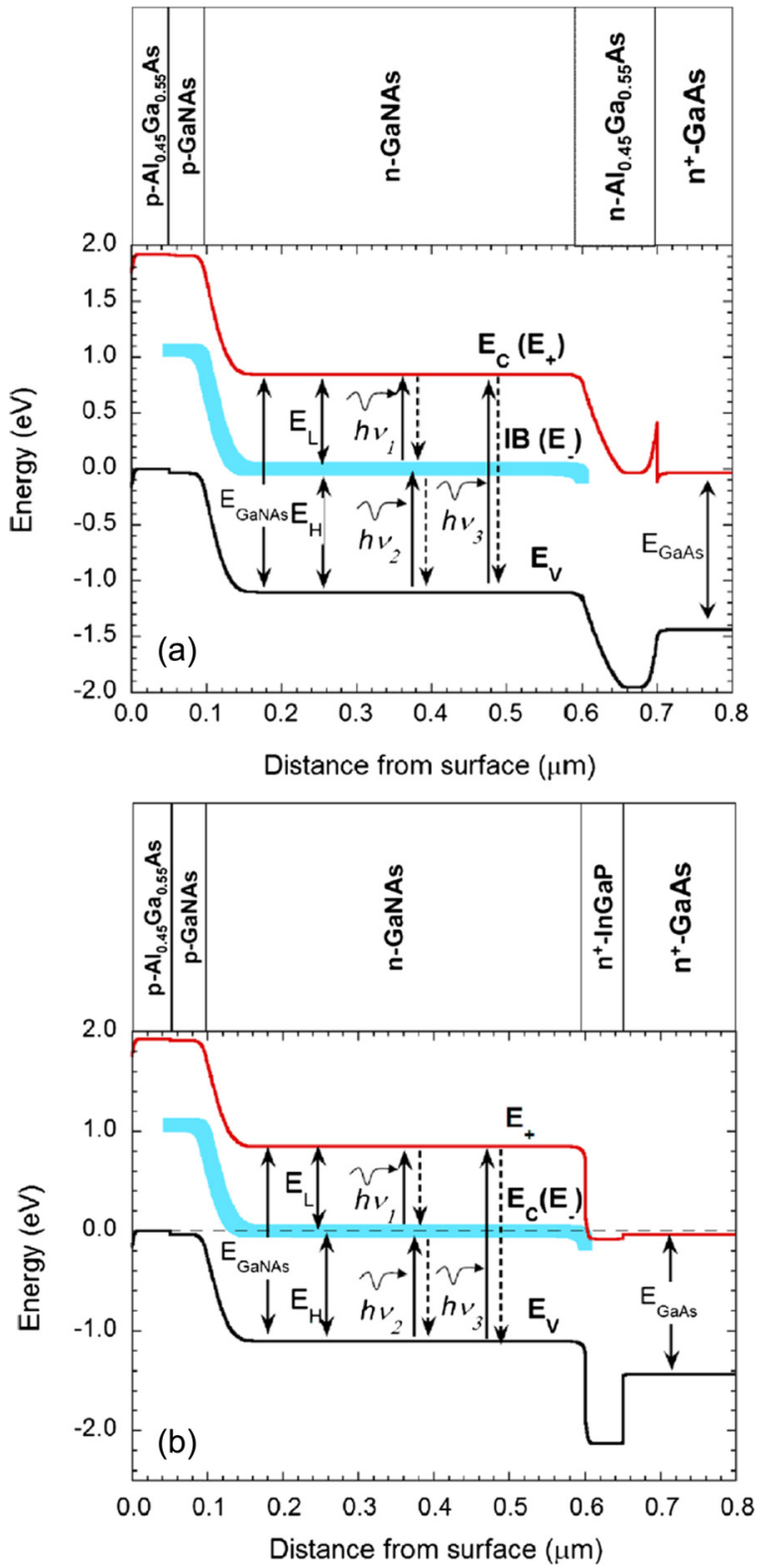

FIG. 16. (a) The structure and band diagram of a BIB device with the IB disconnected from the contacts. Transitions generating electron-hole pairs utilizing the IB are denoted as $h \nu_{1}, h \nu_{2}$. The transitions from VB to CB are represented by $h \nu_{3}$. (b) The structure and the band diagram of an UIB device with the IB connected to the backside contact. Reprinted with permission from López et al., Phys. Rev. Lett. 106, 028701 (2011). Copyright 2011 American Physical Society.

device, this contribution is reduced in the BIB. The main contribution to the BIB photocurrent is dominated by the GaNAs VB-CB transitions, and clarifies that GaNAs is responsible for the transport of the photo-excited carriers. Additional support for the electron transport in the GaNAs $\mathrm{CB}$ has been provided by a comparative study of electroluminescence (EL) on both of the devices whose details are described in the original paper. ${ }^{83}$ Compared to a single EL peak in the UIB at $1.15 \mathrm{eV}$ due to VB-IB recombination, another peak appeared at the low energy side in the BIB at $0.9 \mathrm{eV}$ due to IB-CB recombination originating from electron injection from the GaAs contact to the GaNAs CB in the BIB device. The presence of the optical transition between 


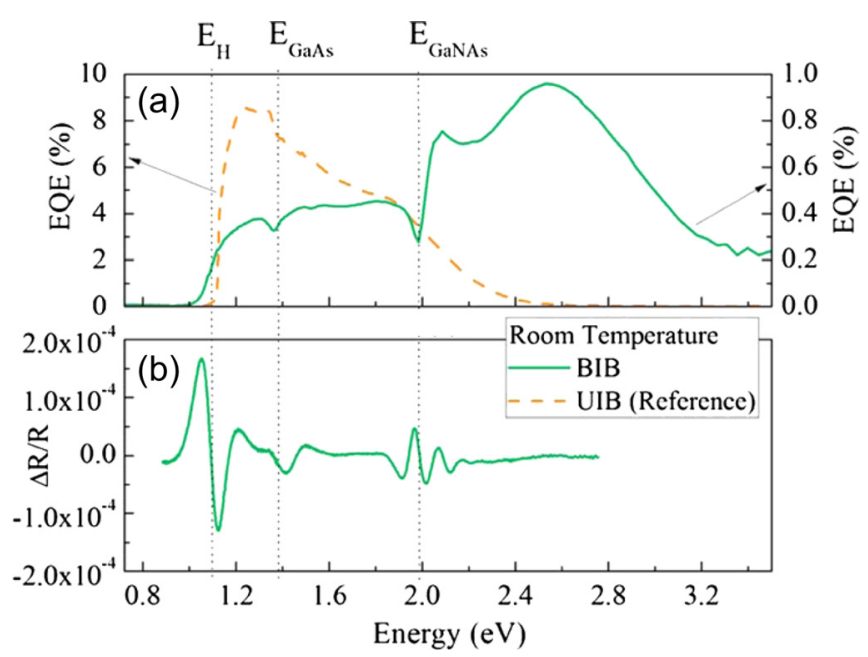

FIG. 17. (a) Spectral dependence of the EQE at RT. The solid line represents the EQE response for the BIB device and the dashed line for the UIB device. (b) PR spectrum of the BIB device. Adapted with permission from López et al., Phys. Rev. Lett. 106, 028701 (2011). Copyright 2011 American Physical Society.

IB and $\mathrm{CB}$ in the BIB device satisfies a key requirement for the operational IBSC. However, in the present BIB device, though reduced yet the VB to IB contribution to the EQE in Fig. 17(a) suggests for the presence of IB electron leakage pathways into the contact layers. In a fully functional IBSC device, perfect electrical isolation of the IB is required to allow selective collection of photo-carriers from the $\mathrm{CB}$ and VB but not from the IB to preserve the voltage.

However, as has been pointed out by Luque and Martí, ${ }^{84}$ the missing link in the above experiments is the absence of a direct observation of photocurrent production by TSPA process. Evidencing photocurrent by TSPA is necessary to demonstrate the validity of the material choice for IBSC application since states existing deep in a semiconductor bandgap typically gives rise to traps and/or recombination centers that severely degrade the photo-voltage.

In the experiments that follow, an evidence for the TSPA effect in GaNAs IBSC structures is presented. ${ }^{85}$ These

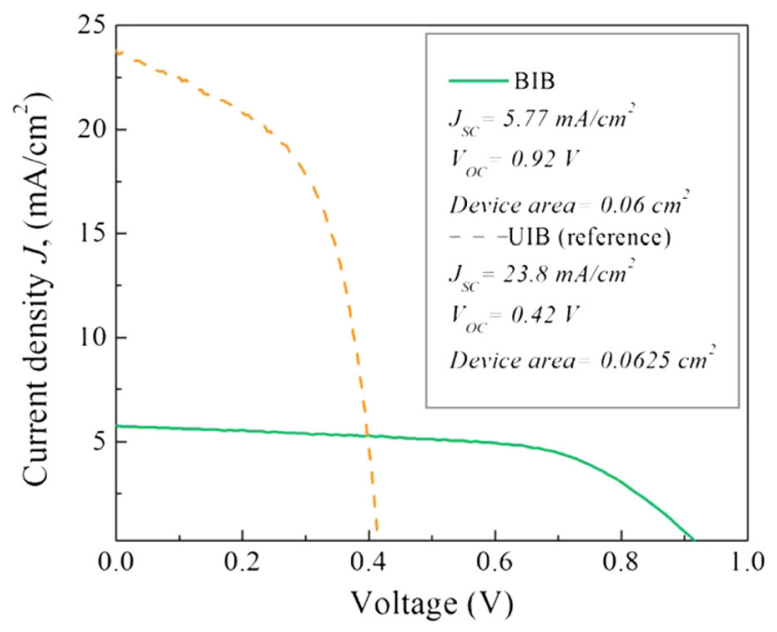

FIG. 18. $J-V$ characteristics measured on BIB (solid line) and UIB (dashed line) devices. Adapted with permission from López et al., Phys. Rev. Lett. 106, 028701 (2011). Copyright 2011 American Physical Society. devices consist of $n$-GaNAs IB layers sandwiched between $p$-type AlGaAs emitters and n-type AlGaAs barrier layers with suitable doping levels. Note that the GaNAs layers are lightly $n$-type doped in order to partially fill the $\operatorname{IB}\left(E_{-}\right)$band to assist low-energy photon absorption between $\operatorname{IB}\left(E_{-}\right)$and $\mathrm{CB}\left(E_{+}\right)$bands. Again, two types of IBSC structures, the UIB and the BIB as shown in Fig. 19(a), were used. Shown in Figs. 19(b) and 19(c) are respective band diagrams calculated using a Poisson solver at dark and zero bias condition. $^{86}$ First in the UIB reference sample, the backside $n$-AlGaAs layer was heavily doped resulting in a thin electrically transparent barrier between the IB in GaNAs layer and the $n$-GaAs substrate. In the second sample, BIB structure, the backside $n$-AlGaAs layer was lightly doped providing a good barrier blocking the electrical contact between the IB and the GaAs substrate. In order to demonstrate the effects

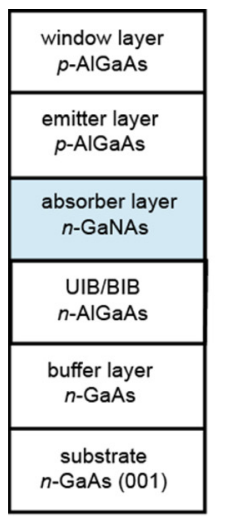

(a)

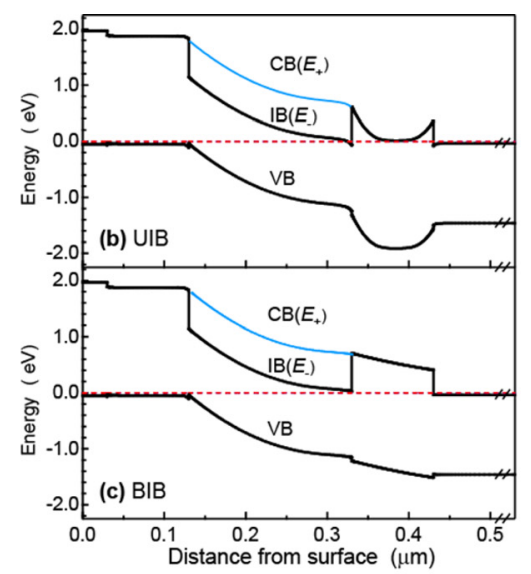

hoton energy (eV)

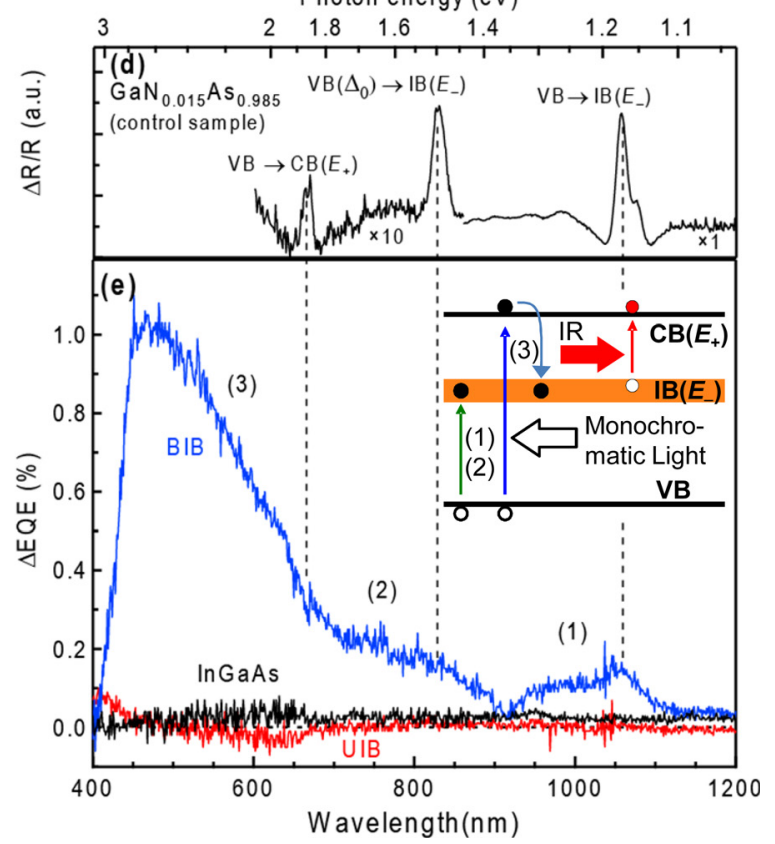

FIG. 19. (a) UIB/BIB structures grown on $n$-GaAs substrates using RF-MBE. In an additional test structure, the GaNAs IB layer of the BIB structure has been replaced by an InGaAs layer with similar bandgap but without an IB. (b) and (c) Calculated band diagrams at RT. (d) PR spectrum of a GaNAs control sample at RT, (e) $\triangle \mathrm{EQE}$ spectra for UIB, BIB, and InGaAs test structures. Reprinted with permission from Ahsan et al., Appl. Phys. Lett. 100, 172111 (2012). Copyright 2012 AIP Publishing LLC. 
of the IB, a test structure was also grown with n-GaNAs layer replaced by $n$-InGaAs which does not have an IB. Structures were grown with radio frequency-plasma assisted MBE (RF-MBE).

Proof for the photocurrent production for the $\operatorname{IB}\left(E_{-}\right)$to $\mathrm{CB}\left(E_{+}\right)$absorption followed by $\mathrm{VB}$ to $\mathrm{IB}\left(E_{-}\right)$absorption has been obtained from the experiment schematically outlined in the inset of Fig. 19(e). Two optical excitations using an IR source for $\mathrm{IB}\left(E_{-}\right)$to $\mathrm{CB}\left(E_{+}\right)$transition and a monochromatic source for other transitions were used in the experiment. The IR light source produced with suitable IR filter which passes AM 1.5 sun light with the wavelength longer than $1400 \mathrm{~nm}$ or the photon energy below $0.88 \mathrm{eV}$. Photons of this energy cannot induce the $\mathrm{VB}$ to $\operatorname{IB}\left(E_{-}\right)$transition that requires energy of more than $1.17 \mathrm{eV}$. However, illumination with these low energy photons from the IR source can pump electrons from $\mathrm{IB}\left(E_{-}\right)$to $\mathrm{CB}\left(E_{+}\right)$. The second, monochromatic source with the photon energy range of $1-4 \mathrm{eV}$ was used to measure the EQE spectra at the short-circuit condition. Under the monochromatic light irradiation, the additional IR bias light illumination enables $\operatorname{IB}\left(E_{-}\right)$to $\mathrm{CB}\left(E_{+}\right)$transitions, resulting in an increased photocurrent through TSPA of electrons from $\mathrm{VB}$ to $\mathrm{CB}\left(E_{+}\right)$via the IB. Therefore, " $\triangle \mathrm{EQE}$ ", the difference of EQE with and without IR illumination, represents the contribution of the photocurrent production through the TSPA process.

Shown in Fig. 19(e) are the $\triangle E Q E$ spectra of the IBSC structures. A finite $\triangle \mathrm{EQE}$ is observed in the BIB structure confirming photocurrent production due to TSPA, while no measurable photocurrent difference is found in the UIB structure. Similar to the case of UIB, no $\triangle \mathrm{EQE}$ has been observed in the $n$-InGaAs test structure. This confirms that in both the UIB and the $n$-InGaAs structures, only the conventional two-band transitions occurred and a TSPA process does not contribute to the photocurrent.

Details of the photocurrent production can be analyzed in terms of three transitions evident in the PR spectrum shown in Fig. 19(e). The $\triangle \mathrm{EQE}$ of the transition (1) [VB to $\left.\mathrm{IB}\left(E_{-}\right)\right]$shows a substantial tail below its edge at $1.17 \mathrm{eV}$. This tail is characteristic of as-grown GaNAs layers ${ }^{79,87}$ and is caused by potential fluctuations in $\operatorname{IB}\left(E_{-}\right)$edge location. This characteristic tail is also visible in transition (2), which involves the electron excitation between spin-orbit split VB and $\operatorname{IB}\left(E_{-}\right)$band of GaNAs, as also observed in the PR spectrum in Fig. 19(d). Transition (3) VB to $\mathrm{CB}\left(E_{+}\right)$occurs in $n$-GaNAs and is very close to $\mathrm{VB}$ to $\mathrm{CB}$ transition in $p$-AlGaAs emitters. The observation of transition (3) in $\triangle E Q E$ spectrum can be explained by efficient excitation of the electrons from the VB to $\mathrm{CB}$ followed by a fast relaxation to the $\operatorname{IB}\left(E_{-}\right)$where the electrons accumulate and can be re-excited to $\mathrm{CB}$ by low energy photons. Comparing to the short wavelength transitions, $\triangle \mathrm{EQE}$ signals in transitions (1) and (2) are much weaker due to the weak optical absorption of long wavelength photons in the thin GaNAs IB layer of the present structures.

One of the key requirements for an efficient IBSC is the perfect electrical isolation of the IB from the contacts allowing selective collection of photo-carriers from the $\mathrm{CB}$ and VB but not from the IB. ${ }^{88,89}$ With this condition, the IB is coupled to the VB and the $\mathrm{CB}$ only through optical transitions. This requires efficient energy barrier layers to block IB electron escape pathways between the IB and the contact layers. Although the importance of blocking layers has been recognized before, there has been missing a systematic study of the layer parameters on the blocking behavior. López et al. used one specific thickness and doping concentration. ${ }^{83}$ Ahsan et al. carried out a systematic study of the effects of the blocking layer parameters of $n$-type AlGaAs on the IBSC performance. They have drawn the barrier design guide by analyzing the electronic structure and electron transport properties in simulation. ${ }^{90}$

As shown in Fig. 20(a), direct tunneling through and thermal excitation over the blocking barrier are major carrier escape pathways reducing the photoinduced occupation of the IB and efficiency of the TSPA resulting in a reduced $V_{\text {OC }}$. In designing the blockage of the carrier escape pathways, a series of experiments were performed on IBSC structures with three different levels of electron doping in the AlGaAs IB barrier layers $\left(4 \times 10^{17} \mathrm{~cm}^{-3}, 4 \times 10^{16} \mathrm{~cm}^{-3}\right.$,
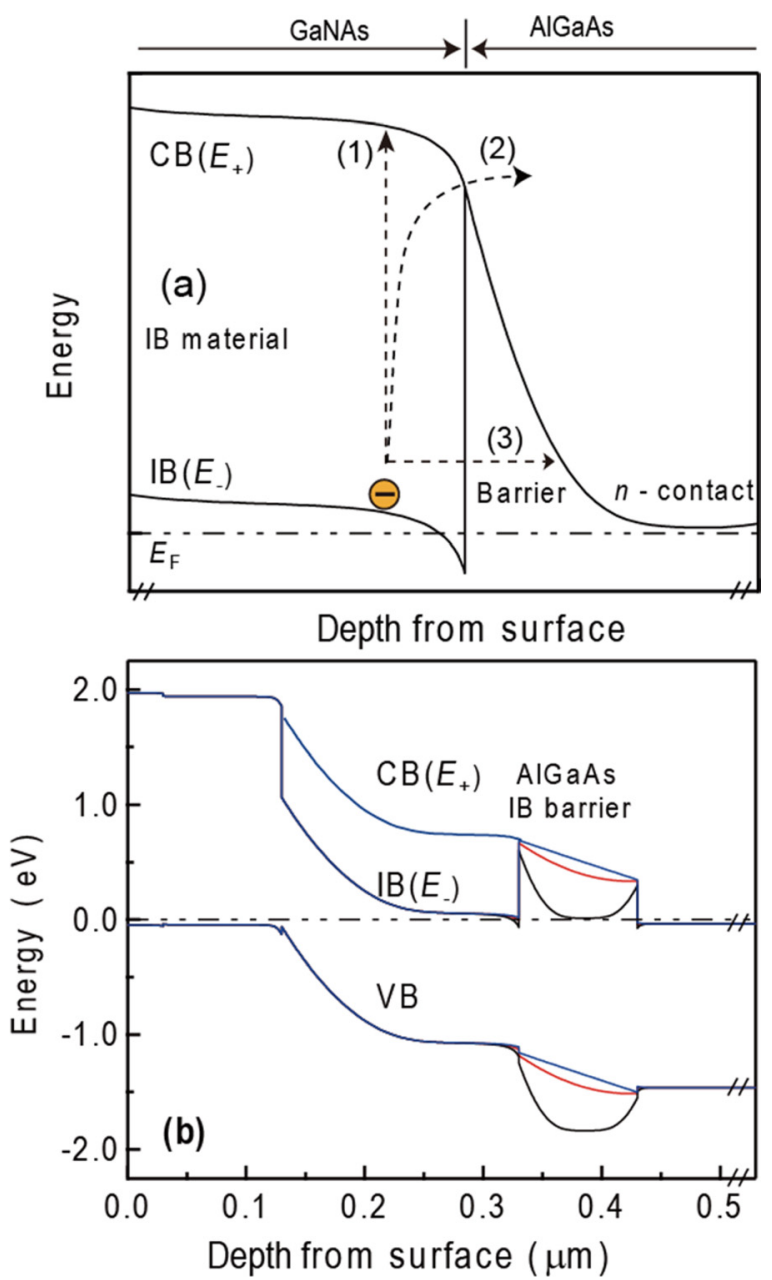

FIG. 20. (a) Schematic illustration of IB electron escape pathways, e.g., (1) optical, (2) thermal, and (3) tunneling toward an $n$-contact layer. Thermal and tunneling escape should be restricted for an operational IBSC. (b) Calculated band diagrams of IBSC structures at dark for three different doping conditions of the backside AlGaAs IB barrier layers. Black, red, and blue lines represent electron doping concentrations of $4 \times 10^{17} \mathrm{~cm}^{-3}$, $4 \times 10^{16} \mathrm{~cm}^{-3}$, and $1 \times 10^{15} \mathrm{~cm}^{-3}$, respectively. The parameters for other layers are kept unchanged. 
and undoped) that allowed different degrees of electrical transparency or blockage of the IB electrons toward the $n$-GaAs contact layers. ${ }^{90}$ The structures are similar as that of Fig. 19(a) except that the electron doping levels in the AlGaAs IB barriers are changed. Figure 20(b) shows the simulated band profiles for three different levels of electron doping in the backside AlGaAs layers $\left(4 \times 10^{17} \mathrm{~cm}^{-3}\right.$, $4 \times 10^{16} \mathrm{~cm}^{-3}$, and $1 \times 10^{15} \mathrm{~cm}^{-3}$ ). The simulated data were calculated using a Poisson solver at dark and zero bias condition. ${ }^{86}$ The electron doping levels used here were taken from measured data in thick Si-doped AlGaAs layers separately grown on semi-insulating GaAs substrates under similar growth conditions. Note that the upper limit of unintentional background doping for the undoped AlGaAs barrier was assumed to be $1 \times 10^{15} \mathrm{~cm}^{-3}$ as it was difficult to measure the carrier concentration below this level in RT Hall measurements. The $\mathrm{CB}$ profile of the heavily doped AlGaAs barrier, represented by black line in Fig. 20(b), shows a very thin triangular potential barrier between IB and the contact. This, in combination with a calculated very high electric field $(300 \mathrm{kV} / \mathrm{cm})$ at the interface, results in an efficient electron tunneling and poor isolation of the IB from the contact. Decreasing level of the electron doping and widening of the triangular barriers results in increasingly efficient blocking of the IB electrons. A nearly flat electric field distribution of around $30 \mathrm{kV} / \mathrm{cm}$ was obtained for the undoped $\mathrm{AlGaAs}$ barriers. Additional effect reducing the charge transfer across the blocking layer originates from the difference in the doping level of AlGaAs barrier and GaNAsSb absorber. Thus, reduction of the $n$-type doping of the AlGaAs barrier from $4 \times 10^{17} \mathrm{~cm}^{-3}$ and $1 \times 10^{15} \mathrm{~cm}^{-3}$ leads to an increase in the effective energy barrier height by almost $100 \mathrm{meV}$ at the GaNAs/AlGaAs interface. This effective increase in energy height can provide an additional blockage for thermal escape pathway of IB electrons.

Figure 21(a) shows the RT $J-V$ characteristics under 1 sun of AM 1.5 spectrum. The $V_{\mathrm{OC}}$ in the IBSC structures are $0.44,0.52$, and $0.80 \mathrm{~V}$, for the $n$-type AlGaAs layers doped to $4 \times 10^{17} \mathrm{~cm}^{-3}, 4 \times 10^{16} \mathrm{~cm}^{-3}$, and undoped, respectively. In solar cells, the $V_{\mathrm{OC}}$ values are related to the quasi-Fermi level difference and are determined by the gap $E_{g}$ between electron and hole collecting bands. The key question in the present case is whether the electron collecting band is $\mathrm{IB}\left(E_{-}\right)$or $\mathrm{CB}\left(E_{+}\right)$. The $\operatorname{VB}-\mathrm{IB}\left(E_{-}\right)$bandgap for the GaNAsSb absorber can be estimated from the onset of EQE response at the long wavelength region around $1.1 \mathrm{eV}$ $(\sim 1140 \mathrm{~nm})$, as observed in the inset of Fig. 21(b). In general, the $V_{\mathrm{OC}}$ is given by $V_{\mathrm{OC}}=\left(E_{g}-\Delta\right) / q$. Here, $q$ is an elemental charge, and the offset $\Delta$ has been shown to be larger than $0.4 \mathrm{eV}$ under 1 sun illumination..$^{91}$ Therefore, in the present IBSC structures the $\Delta$ values with respect to VB$\mathrm{IB}\left(E_{-}\right)$gaps are $0.66,0.58$, and $0.30 \mathrm{eV}$, respectively, with decreasing level of barrier doping. This suggests that the $V_{\mathrm{OC}}$ cannot be attributed to the $\operatorname{VB}-\operatorname{IB}\left(E_{-}\right)$bandgap of $1.1 \mathrm{eV}$, but with the decreasing $n$-type doping levels the more effective blocking of the $\operatorname{IB}\left(E_{-}\right)$leads to an effective collection of electrons photoexcited to $\mathrm{CB}\left(E_{+}\right)$band and increasingly larger role of the $\mathrm{VB}-\mathrm{CB}\left(E_{+}\right)$bandgap in determining the $V_{\mathrm{OC}}$.
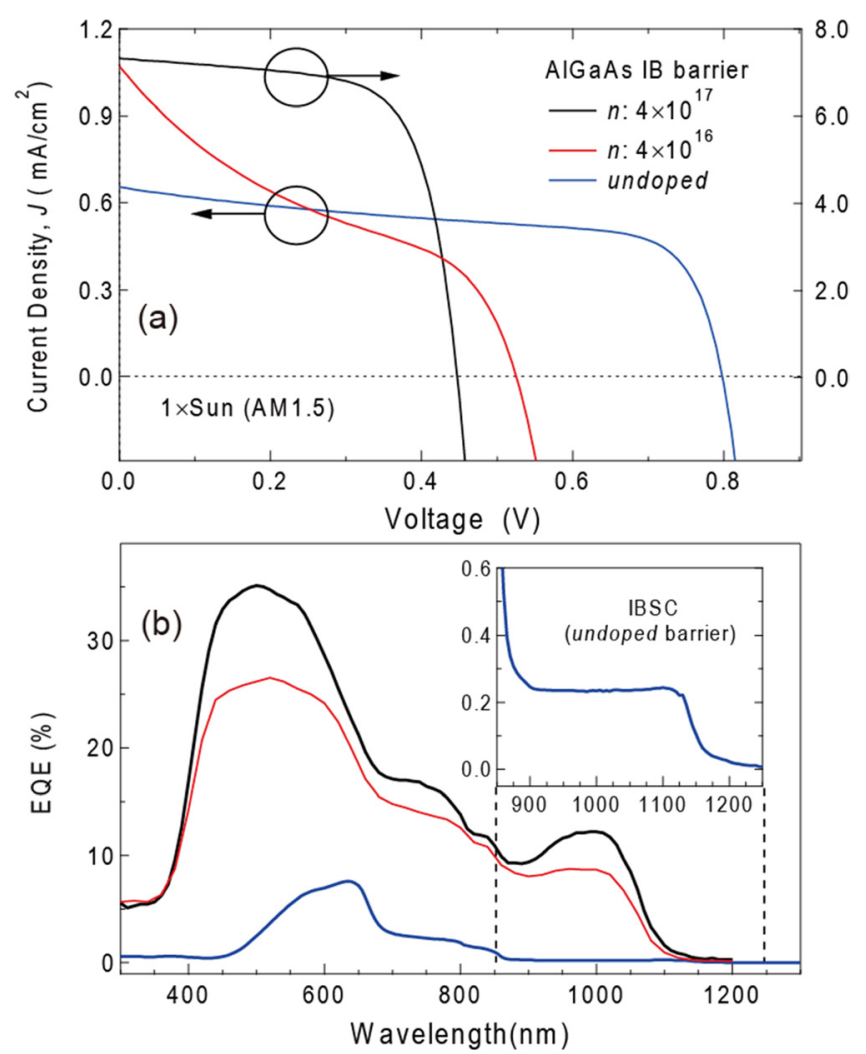

FIG. 21. (a) $J-V$ characteristics under 1 sun illumination of AM 1.5 and (b) spectral dependence of EQE at RT. Black, red, and blue lines represent IBSC structures for the backside AlGaAs electron doping levels of $4 \times 10^{17} \mathrm{~cm}^{-3}, 4 \times 10^{16} \mathrm{~cm}^{-3}$, and undoped barrier, respectively. Inset in (b) shows the enlarged EQE response for the undoped barrier device around the long wavelength $\mathrm{VB} \rightarrow \mathrm{IB}\left(E_{-}\right)$transition (around $1140 \mathrm{~nm}$ or $1.1 \mathrm{eV}$ ).

An apparent reduction in the photocurrent production has been observed in Fig. 21(a) with decreasing barrier doping levels. The $J_{\mathrm{SC}}$ values are $7.32,1.06$, and $0.65 \mathrm{~mA} / \mathrm{cm}^{2}$, respectively, with decreasing level of barrier doping. This is likely due to an inefficient collection of the electrons photoexcited to the $\mathrm{CB}\left(E_{+}\right)$band. Details of the photo-carrier collection are illustrated in Fig. 21(b), in which EQE spectra of the IBSC structures at RT are shown. Note that the EQE magnitudes reduce with the decrease of barrier doping level especially at longer wavelengths. This is an expected behavior as the more efficient blocking layer reduces collection of electrons photoexcited to the $\operatorname{IB}\left(E_{-}\right)$with the long wavelength photons.

The EQE spectra discussed so far were measured at a short-circuit condition by maintaining a zero external bias. Carrier collection can be enhanced with an aid of an electric field by applying reverse biases when the extraction of photo-generated carriers is incomplete. ${ }^{92,93}$ Figure 22(b) shows EQE spectra of the IBSC with undoped barrier with increasing reverse bias from $+0.5 \mathrm{~V}$ to $-1.5 \mathrm{~V}$. Compared with the long wavelength $\mathrm{EQE}$ responses, much enhancement of the short wavelength EQE responses can be observed. To examine the transitions contributing to the short wavelength photon absorptions, PR spectroscopy was measured at RT on an undoped GaNAs control sample of similar $\mathrm{N}$ composition. The features in the PR spectrum in Fig. 22(a) consist of four transitions: three from the GaNAs 


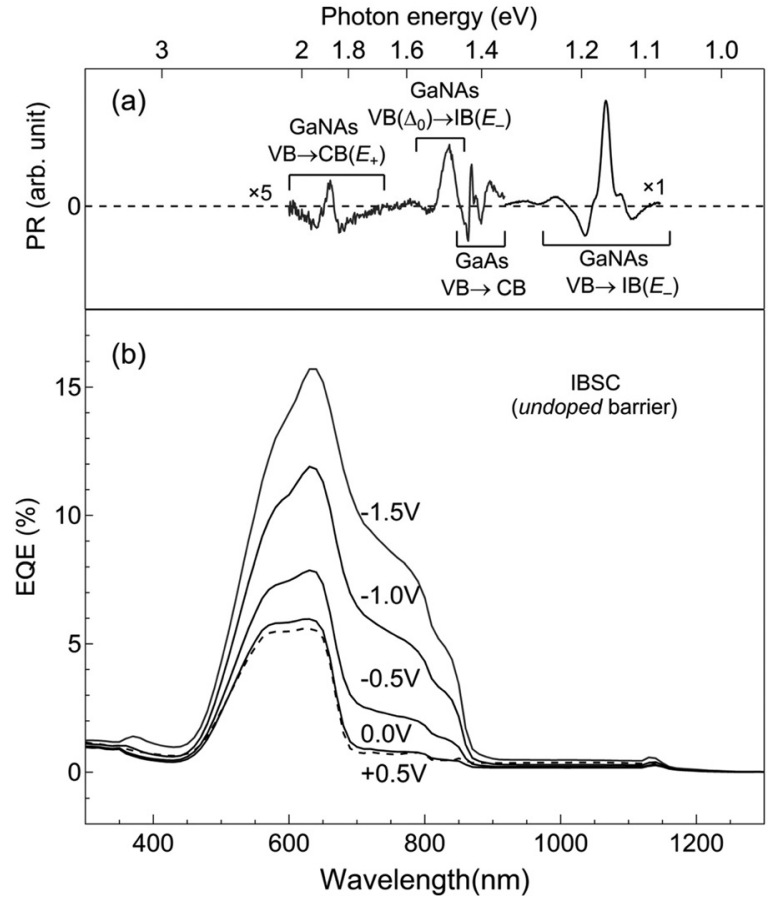

FIG. 22. (a) PR spectrum at RT of a GaNAs control sample (N content is $1.75 \%$ ). (b) EQE spectra of the IBSC with an undoped barrier, measured at RT. With decreasing biases from $+0.5 \mathrm{~V}$ (dashed line) to $-1.5 \mathrm{~V}$ (solid lines), an overall enhancement of the EQE can be observed. $\mathrm{CB}\left(E_{+}\right)$carrier collection, which is otherwise inhibited by poor diffusion length, is enhanced when assisted by an electric field. The apparently low $J_{\mathrm{SC}}$ in Fig. 21(a) is due to the low EQE response at $0 \mathrm{~V}$.

layer and one from the GaAs substrate. This indicates that the dominant contribution to $\mathrm{EQE}$ signals originates from the GaNAs VB $\rightarrow \mathrm{CB}\left(E_{+}\right)$transitions wavelength shorter than $660 \mathrm{~nm}$ (above $1.88 \mathrm{eV}$ ) superimposed with those from $p$ AlGaAs emitter. The onset of mid-wavelength $\mathrm{EQE}$ signal at about $870 \mathrm{~nm}(1.42 \mathrm{eV})$ matches the GaAs signals in PR spectrum in Fig. 22(a). This suggests for the field-assisted collection of minority carriers (holes) from $n$-GaAs contact, which were otherwise likely lost in the GaNAs flat-band area. Another PR feature observed in GaNAs originates from spin-orbit split $\mathrm{VB}\left(\Delta_{0}\right)$ to $\mathrm{IB}\left(E_{-}\right)$transitions. However, similarly to the case of $\mathrm{VB} \rightarrow \mathrm{IB}\left(E_{-}\right)$transitions, it is likely that the contribution from $\operatorname{VB}\left(\Delta_{0}\right) \rightarrow \mathrm{IB}\left(E_{-}\right)$transitions to the $\mathrm{EQE}$ is equally low due to the increased blocking efficiency of the undoped barrier.

The reason for the large difference in the long wavelength EQE responses in Fig. 22(b) of the IBSC of medium doped barrier $\left(4 \times 10^{16} \mathrm{~cm}^{-3}\right)$ compared to that of undoped case is not fully clear. One likely interpretation is the additional presence of carrier leakage path through localized states of the $\operatorname{IB}\left(E_{-}\right)$close to the original nitrogen level. The importance of these states at $\sim 1.6 \mathrm{eV}$ above VB has been shown in transient photocapacitance study performed on GaInNAsSb-based solar cells. ${ }^{94}$ The degree of tunneling escape of IB carriers that are thermally excited into these states will be highly sensitive to the thickness of the triangular potential, and may result in the long wavelength carrier collection when barriers are highly doped, and thin.

The above results have shown that blocking of the charge transport in the IB is the key requirement for the operation of IBSC, in which the best blocking properties were achieved for $100 \mathrm{~nm}$-thick undoped AlGaAs barriers (unintentionally doped $\sim 10^{15} \mathrm{~cm}^{-3}$ ) in experiments. This is in good agreement with the optimal blocking properties achieved for lightly doped $\left(<10^{16} \mathrm{~cm}^{-3}\right)$ AlGaAs barriers thicker than $60 \mathrm{~nm}$ in simulation. ${ }^{90}$

While the direct observation of photocurrent production due to TSPA in GaNAs HMAs demonstrates the potentials of this system for thin-film IBSC application, the amount of photocurrent is still very low. This can be ascribed to the degraded electrical and optical qualities of this system studied extensively since the early 1990s. With increasing N composition, the severe deterioration of the material properties leads to short minority-carrier diffusion lengths and poor carrier extraction in GaNAs single-junction solar cells compared with that of the host GaAs material. ${ }^{41}$ The coexistence of different local environments in the alloy complicates the electronic properties of GaNAs generating band fluctuations. These environments result in deep carrier trap states having a few tens to a few hundreds meV of localization energies. The use of antimony ( $\mathrm{Sb}$ ) has been shown effective in improving material qualities of GaInNAs QWs and thin films. ${ }^{78,79}$

Ahsan et al. have carried out a comparative study of TSPA experiments between a GaNAs and a GaNAsSb IBSC. They show that an improvement in the photocurrent production has been observed in the GaNAsSb IBSC due to improved material quality. ${ }^{95}$ The structures are similar as that of Fig. 19(a) except that during the growth of GaNAs absorber layer with $\mathrm{Sb}$, the $\mathrm{Sb}$ beam flux was set to $1.0 \times 10^{-8}$ Torr by fixing a cracker valve. The $\mathrm{N}$ fluxes were kept constant in both of the structures. Also, the Si dopant concentrations in both of the structures were kept constant at $5 \times 10^{17} \mathrm{~cm}^{-3}$.

The $\triangle \mathrm{EQE}$ spectra of the TSPA experiments are shown in Fig. 23(a). A distinct improvement in the $\triangle \mathrm{EQE}$ of the GaNAsSb IBSC structure is observed compared to the GaNAs IBSC. In both of the IBSC structures, the excess electrons trapped in the $\mathrm{IB}\left(E_{-}\right)$are re-excited to $\mathrm{CB}\left(E_{+}\right)$ with IR photons producing an additional photocurrent that effectively results from two photons producing one electronhole pair. The inset in Fig. 23(a) is an expanded viewgraph in $800-1200 \mathrm{~nm}$ range of wavelength with the $\triangle \mathrm{EQE}$ values in the logarithmic scale. Any degradation of the $\triangle \mathrm{EQE}$ values was not observed despite an increased amount of nitrogen in the GaNAsSb absorber layer. Instead, the enhancement in the $\triangle \mathrm{EQE}$ value of the GaNAsSb IBSC above the $\mathrm{VB} \rightarrow \mathrm{CB}\left(E_{+}\right)$transitions by a factor of 2 suggests improved $\mathrm{IB}\left(E_{-}\right) \rightarrow \mathrm{CB}\left(E_{+}\right)$transitions with IR photons producing an additional photocurrent.

The improved photocurrent production due to TSPA in the GaNAsSb IBSC can be attributed to the enhanced $\mathrm{IB}\left(E_{-}\right) \rightarrow \mathrm{CB}\left(E_{+}\right)$transitions. Theoretically, a suitable electron filling of the IB is expected to increase both of the sub-bandgap transitions at a high rate, ${ }^{88}$ and an increased photocurrent can be achieved. In fact, an increased carrier population has been deduced from PR studies in the GaNAsSb $\mathrm{IB}\left(E_{-}\right)$band compared with that of the GaNAs absorber, and can be attributed to the enhancement of the 

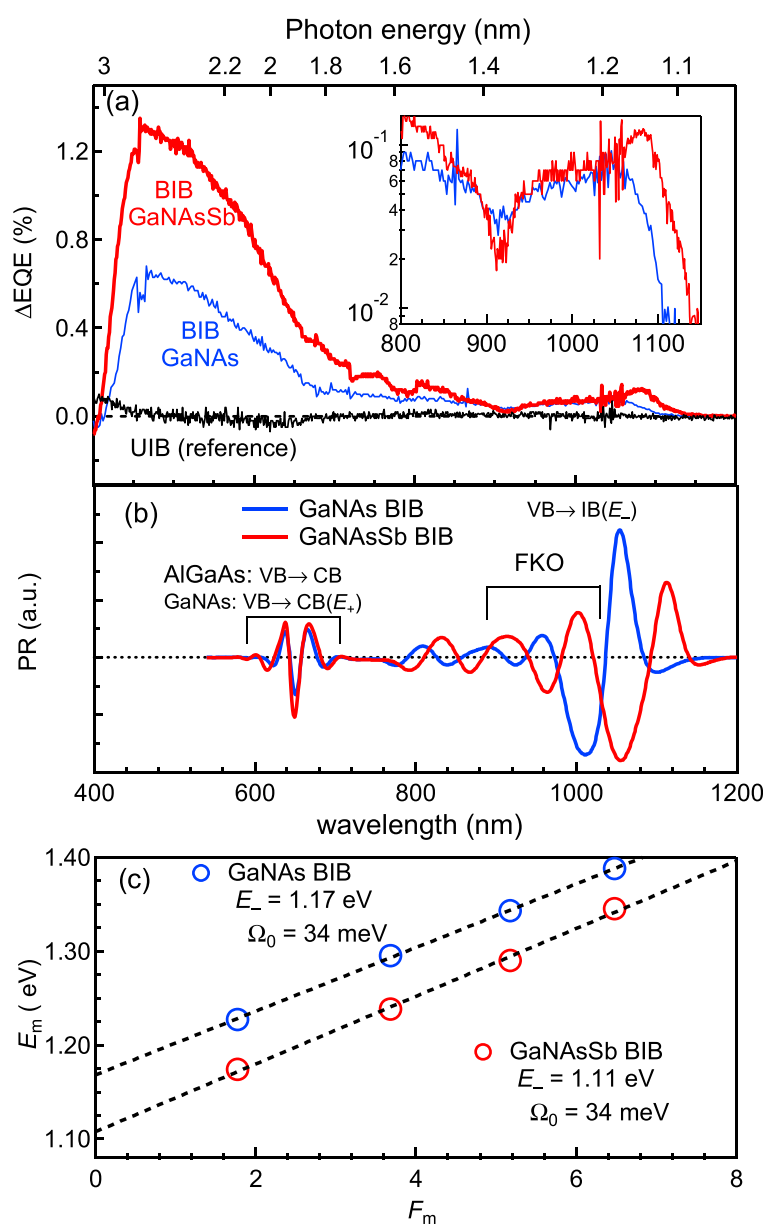

FIG. 23. RT measurements: (a) spectral dependence of $\triangle \mathrm{EQE}$ of GaNAs and GaNAsSb BIB structures, respectively. For reference, the $\triangle \mathrm{EQE}$ spectrum of a GaNAs UIB structure is also shown in (a). (b) PR spectra of both structures at RT. (c) Linear plot of the $E_{m}$ of FKOs around $\mathrm{IB}\left(E_{-}\right)$transitions as a function of $F_{m}$, which is a reduced parameter for the number $(m)$ of the extrema $E_{m}$

improved $\mathrm{IB}\left(E_{-}\right) \rightarrow \mathrm{CB}\left(E_{+}\right)$transitions with IR photons producing an additional photocurrent.

Figure 23(b) shows the PR spectra of the two structures at RT. The structures in the long wavelength regions are related to the GaNAs $\mathrm{VB} \rightarrow \mathrm{IB}\left(E_{-}\right)$transitions. The oscillatory features above the $\mathrm{VB} \rightarrow \mathrm{IB}\left(E_{-}\right)$transitions are the Franz-Keldysh oscillations (FKO) due to the presence of strong electric field in the depleted $\mathrm{GaNAs}(\mathrm{Sb})$ layers. The high energy features above $1.80 \mathrm{eV}$ are most likely superimposed peaks of $\mathrm{VB} \rightarrow \mathrm{CB}\left(E_{+}\right)$and the interband transitions in $p$-AlGaAs emitters having similar energies. An indirect method for determining the bandgap energy and doping concentrations is employed using the FKO features in Fig. 23(b). ${ }^{96}$ Shown in Fig. 23(c) is a linear fit to the energy positions of extrema $\left(E_{m}\right)$ of the FKOs taken below $1.40 \mathrm{eV}$ of photon energy to avoid overlapping PR signals from the top $p$-GaAs contact layers. The intercept of the fit gives the band edge $E_{g}$ and the slope gives $\hbar \Omega$, a characteristic energy associated with the bandgap, and related to the electric field $F$ that can give an estimation of ionized dopants, and hence the carrier concentration. ${ }^{96}$ The obtained values of the $\mathrm{IB}\left(E_{-}\right)$band edges for GaNAs and GaNAsSb absorbers are 1.17 and $1.11 \mathrm{eV}$, respectively. The key observation is that an improved electron concentration in the GaNAsSb layer is obtained by a factor of 1.2 compared with that of the GaNAs layer. Note that a constant amount of Si dopants was provided to each of the layers. This increase in the effective carrier concentration in the GaNAsSb layer despite an increase in the $\mathrm{N}$ composition resulting in a lowered $\operatorname{IB}\left(E_{-}\right)$band edge, can be ascribed to the surfactant effect of the Sb during growth of GaNAs layer. ${ }^{78,79}$ This increased carrier population in the GaNAsSb $\operatorname{IB}\left(E_{-}\right)$band is attributed to the extra carriers enhancing the $\mathrm{IB}\left(E_{-}\right) \rightarrow \mathrm{CB}\left(E_{+}\right)$transitions with IR photons that produces an additional photocurrent compared with that of the GaNAs absorber without Sb.

\section{II-VI ZnTeO-based thin-film IBSC}

In recent years, as mentioned earlier, II-VI ZnTeO is another HMA system besides III-V GaNAs system which is increasingly drawing interest for IBSC application. This is because of the formation of O-derived narrow band $\left(E_{-}\right)$of extended states located well below the $\mathrm{CB}\left(E_{+}\right)$edge of the $\mathrm{ZnTe}$, and the $E_{-}$band can be used as an IB. ${ }^{54,64,65,97,98}$ In order to study the role of IB on the photocurrent production, there are a few experimental results on TSPA reported using laser or light emitting diode as illumination light sources. ${ }^{64,97}$

Very recently, more detailed studies on the TSPA process in $\mathrm{ZnTeO}$ have been reported by Tanaka et al. ${ }^{98,99}$ They prepared a series of $\mathrm{ZnTeO}$ IBSCs ${ }^{99}$ with different thicknesses of surface-side blocking layer (undoped ZnTe layers with $0,50,100$, and $200 \mathrm{~nm}$ of thicknesses) while other layers were kept fixed, as shown in Fig. 24(b). The UIB structure has the IB connected to the $\mathrm{CB}$ of $\mathrm{ZnO}$ window layer, while BIB has the blocking layers (undoped ZnTe layers) on both the surface and the substrate sides. The UIB device serves the purpose of a reference sample. An additional $\mathrm{ZnTe}$ reference solar cell without a $\mathrm{ZnTeO}$ absorber was prepared as an IB-free reference sample, as shown in Fig. 24(a). $J-V$ curves of these devices under 1 sun illumination (AM 1.5), and the EQE spectra at RT are shown in Figs. 25(a) and 25(b). Note that the $V_{\mathrm{OC}}$ and $J_{\mathrm{SC}}$ values in Fig. 25(a) are higher than those for the devices with a thinner blocking layer. This has been ascribed to the increased fraction of light absorption by the thick ZnTe blocking layers, not by the ZnTeO layers. The smaller bandgap between VB and $\mathrm{IB}\left(E_{-}\right)$of $\mathrm{ZnTeO}$ is likely responsible for the smaller $V_{\text {OC }}$ of UIB device, since the low energy photocurrent edge in UIB at about $1.65 \mathrm{eV}$ in Fig. 25(b) corresponds to the optical transitions from $\mathrm{VB}$ to $\mathrm{IB}$. The second edge at about $2.3 \mathrm{eV}$ in UIB and BIB devices can be attributed to the transitions from the $\mathrm{VB}$ to $E_{+}$in $\mathrm{ZnTeO}$ and from the $\mathrm{VB}$ to the $\mathrm{CB}$ in $\mathrm{ZnTe}$. Further, note that the $V_{\mathrm{OC}}$ values are much lower for these devices compared with the bandgap of ZnTe. This has been ascribed mainly due to the large CB offset existing at the interface between $\mathrm{ZnO}$ and $\mathrm{ZnTeO}$. In these contexts, it is not clear about the role of photocurrent due to TSPA on the increase of $J_{\mathrm{SC}}$ and $V_{\mathrm{OC}}$ with increasing blocking layers.

A direct evidence of photocurrent production via TSPA in these II-VI ZnTeO devices has been provided using an additional IR light source similar to the EQE measurement 

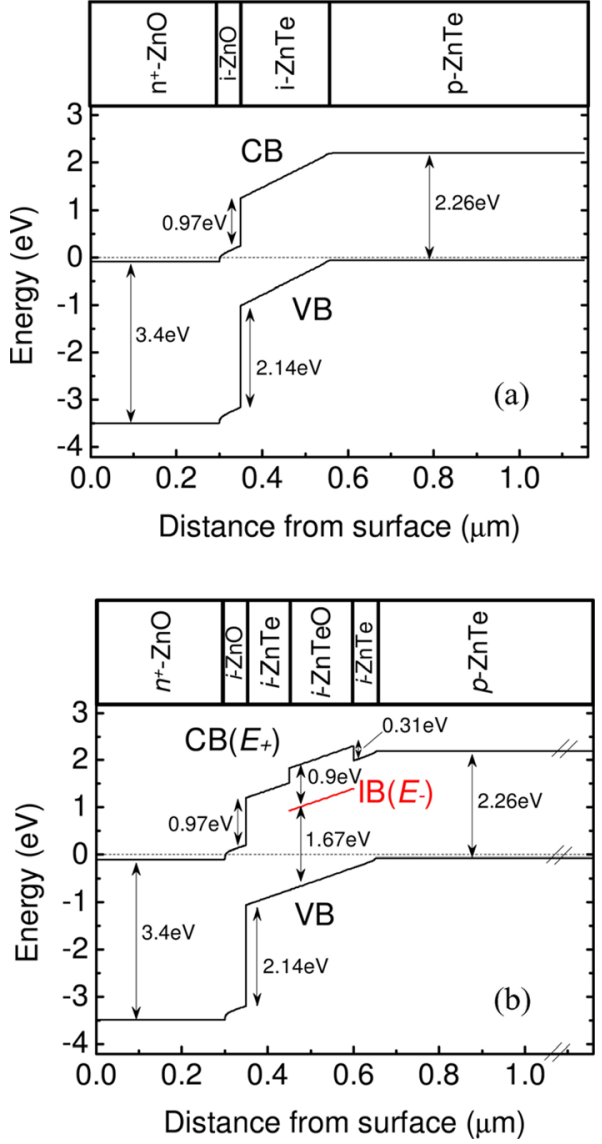

FIG. 24. Device structure and calculated band diagrams of (a) $n^{+}-\mathrm{ZnO} /$ $i-\mathrm{ZnO} / i-\mathrm{ZnTe} / p-\mathrm{ZnTe}$ reference device and (b) $\mathrm{ZnTeO} \mathrm{BIB}$ device. Reprinted with permission from Tanaka et al., IEEE J. Photovoltaics 4, 196 (2014). Copyright 2014 IEEE.

setup discussed in Sec. III A 2. In the present system, the IR light source passes photons with the wavelength longer than $1000 \mathrm{~nm}$ or the photon energy below $1.24 \mathrm{eV}$. The EQE was recorded with and without the IR illumination, and the $\triangle \mathrm{EQE}$ represents photocurrent production due to TSPA. The $\triangle \mathrm{EQE}$ at $\mathrm{RT}$ for the $50 \mathrm{~nm}$-thick barrier device is shown in Fig. $26^{98}$ The increases of $\triangle \mathrm{EQE}$ at the energy region between 1.65 and $2.25 \mathrm{eV}$ are ascribed to the enhanced IB$\mathrm{CB}$ transitions of electrons excited from VB to IB; whereas those at the energy region above $2.25 \mathrm{eV}$ can be explained by the re-excitation of electrons being trapped into IB after the initial transition from VB to $\mathrm{CB}$ and then to IB by a fast relaxation.

While the above experiments demonstrated that the potentials of $\mathrm{ZnTeO}$ alloy for the IBSC application, the performance as a solar cell device is yet low. Improvement of the front-side barrier layer is needed since the present structures require thick $i$-ZnTe barrier layers to isolate the IB from the contacts. As discussed, the thick $i$-ZnTe barrier layer can absorb the crucial part of the solar spectrum which would otherwise be absorbed and converted into electricity by the $\mathrm{ZnTeO}$ base layer. Another key issue is the sufficient collection of photo-carriers due to the sub-bandgap photons. In a real device, the location of the junction existing near the surface poses difficulty in carrier collection for sub-bandgap response where absorption is located farther from the
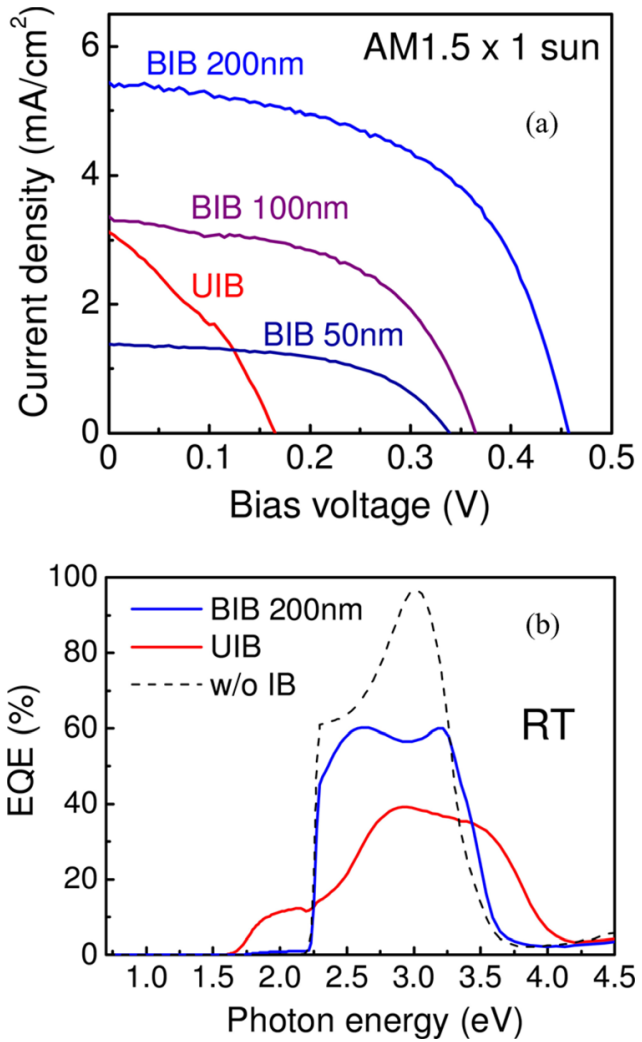

FIG. 25. (a) $J-V$ characteristics of an UIB device and a set of BIB devices with different blocking layer thicknesses $(50,100$, and $200 \mathrm{~nm}$ ). (b) Spectral dependence of EQE for the UIB and the BIB with $200 \mathrm{~nm}$-thick barrier. $\mathrm{EQE}$ spectrum for $\mathrm{ZnO} / \mathrm{ZnTe}$ solar cell without IB is also shown for comparison. Adapted with permission from Tanaka et al., IEEE J. Photovoltaics 4, 196 (2014). Copyright 2014 IEEE.

surface. Phillips et al. investigated an inverted device structure that places the junction near the substrate interface, ${ }^{100}$ using an $n$-ZnSe buffer layer and a graded $n$-ZnSeTe alloy, as shown in Figs. 27(a) and 27(b). The graded ZnSeTe layer is approximately $100 \mathrm{~nm}$-thick and is intended to reduce dislocation formation in transitioning from the $\mathrm{ZnSe}$ lattice constant to the ZnTe lattice constant. Figure 27(c) shows the $J-V$ characteristics of the device. The performance of the inverted structure with $\mathrm{ZnTeO}$ on $\mathrm{GaAs}$ exceeds the performance of a $\mathrm{ZnTe}$ solar cell which is a reference sample

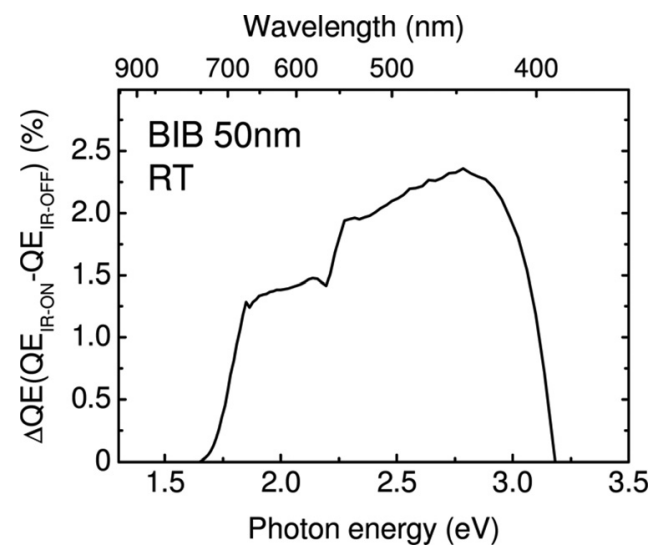

FIG. 26. Spectral dependence of $\triangle \mathrm{EQE}$ measured at RT for the BIB device with a $50 \mathrm{~nm}$-thick blocking layer. Adapted with permission from Tanaka et al., IEEE J. Photovoltaics 4, 196 (2014). Copyright 2014 IEEE. 
(a)

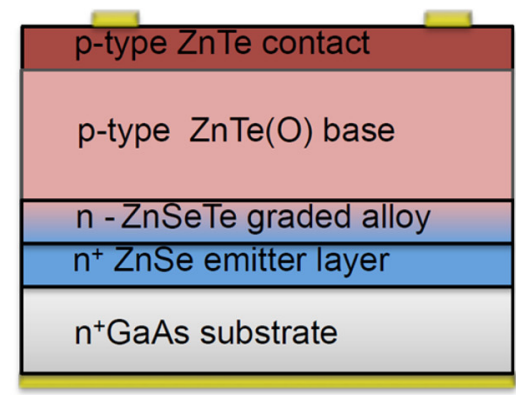

(b) $p^{+} \mathrm{ZnTe}$
contact

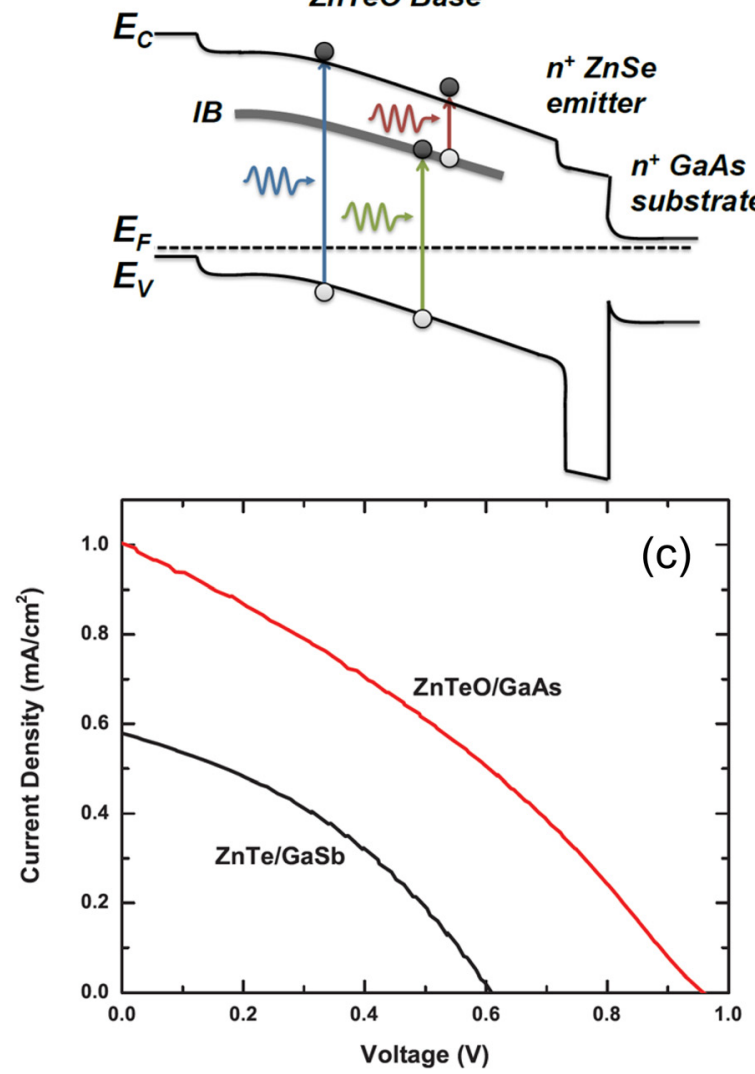

FIG. 27. (a) Device structure and (b) energy band diagram of an inverted $\mathrm{ZnTeO}$ IBSC. (c) $J-V$ characteristics under 1 sun illumination (AM 1.5) of an inverted IBSC structure $(\mathrm{ZnTeO} / \mathrm{GaAs})$ and a homoepitaxially grown $\mathrm{ZnTe} / \mathrm{GaSb}$ reference solar cell. Reprinted with permission from Phillips et al., "IB solar energy conversion in $\mathrm{ZnTeO}$," in Proceedings of the 39th IEEE Photovoltaic Specialists Conference (2013), p. 1640. Copyright 2013 IEEE.

grown homoepitaxially on a GaSb substrate. The performance exceeded despite the presence of large lattice mismatch from GaAs substrates and the associated increase in dislocation density. This behaviour demonstrates the importance of the $n-\mathrm{ZnSe} / p$-ZnTe junction in determining diode performance, where the graded $\mathrm{ZnSeTe}$ layer appears to be desirable for improving the electrical junction. Another key observation for the inverted diode structure is that the performance is maintained using a $\mathrm{ZnTeO}$ base layer, implying that the solar cell performance is not degraded. They have observed sub-bandgap response in the $J-V$ characteristics in the inverted structure using a red laser $(\sim 1.95 \mathrm{eV})$. Because this energy is smaller than the bandgap of ZnTeO, this experiment suggests for the presence of an optically active IB that contributed to the additional photocurrent generation. In order to realize an operational IBSC, carrier leakage pathways from the IB should be blocked to assist photocurrent production due to TSPA.

In short, HMAs are still in the experimental stages of their development. However, the two systems reviewed so far clearly have demonstrated the potentials for IBSC application. For the highly efficient IBSCs, further improvement of the electronic and optical qualities, e.g., mobility and minority carrier lifetime, are required. Lattice-matched systems can allow superior heterojunctions suppressing formation of nonradiative defects, etc. Also, for the electrical isolation of the IB, the device design requires some innovative ideas including material development. Another issue is the improvement of the transition strength of long wavelength photons stepping into the IB. One would expect that if the localized impurity level lies below the $\mathrm{CB}$, the final transition may be stronger due to the more impurity derived $E_{-}$ and more extended like $E_{+}$. This has motivated the research on II-VI ZnTeO system, and recently on $\mathrm{GaN}_{x} \mathrm{P}_{y} \mathrm{As}_{1-x-y}$ system. ${ }^{101,102}$

\section{B. Quantum dot arrays}

\section{Fabrication techniques of multi-stacking QDs layers}

QD or QW superlattice (SL) incorporated in the active region of $p-i-n$ single-junction solar cells has been considered as one of the most promising candidates to realize the IBSCs. ${ }^{2,3,103}$ In the last decade, there has been an extensive effort to demonstrate the QDSCs with a central focus on III-V compound semiconductors. ${ }^{34,104-141}$ For the QDIBSC, QDs are required to be homogeneous and small in size and to be regularly and tightly placed in the active region of the cell as illustrated in Fig. 28, in order to form of an IB or a SL miniband that is well separated in energy from the higher states. ${ }^{142}$ Additionally, the dense QDs must be required to achieve the sufficient photo-absorption in QD layers. The most commonly used fabrication technique of QD structure is to leverage spontaneous self-assembly of coherent three-dimensional (3D) islands in latticemismatched heteroepitaxy known as the Stranski-Krastanov (S-K) growth mode on MBE or MOVPE. The InAs/GaAs heteroepitaxial system has a lattice mismatch of $7.2 \%$, lattice strain introduced by a large mismatch will drive into $\mathrm{S}-\mathrm{K}$ growth. The InAs 2D layer called wetting layer is grown in the initial growth step, and then the growth mode transits into $3 \mathrm{D}$ coherent island growth with increased in the InAs coverage. ${ }^{143}$ The typical areal density of QDs is in order of $10^{10} \mathrm{~cm}^{-2}$ in a single layer for InAs QDs grown on GaAs (001) substrate. However, the density is much less than required for QD-IBSC as described in Sec. III B 4. The QD size and density are dependent on the growth conditions such as the V/III flux ratio, substrate orientation, growth temperature, and material of buffer layer. ${ }^{115,144,145}$ Yamaguchi et al. have demonstrated that the density of QDs is increased by irradiating $\mathrm{Sb}$ to buffer or wetting layer. ${ }^{146-148}$ The ultra-high density of $1 \times 10^{12} \mathrm{~cm}^{-2}$ in the single layer was achieved in their studies. 
(a)

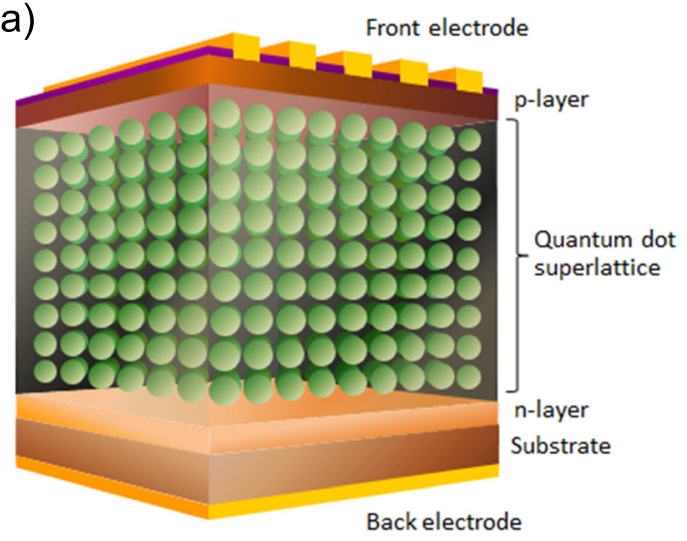

(b)

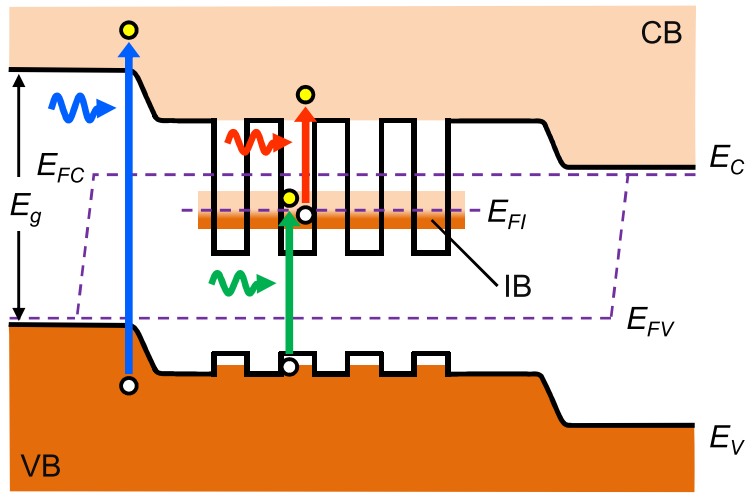

FIG. 28. (a) Schematic structure of QD-IBSC and (b) schematic energy band diagram of QD-IBSC shown with possible photo-absorption and recombination processes involved. The energy gap of host material and quasi-Fermi level of IB (QDs) are given by $E_{\mathrm{g}}$ and $E_{F_{1}}$, respectively.

For further increasing of QDs density, the fabrication of multi-stacking configuration should be needed. However, a gradual build-up of internal lattice strain with increased number of stacking results in an increase in both the size and its fluctuation ${ }^{149}$ for the multi-stacked QDs grown by S-K growth mode. In addition, the excess-accumulated strain has the negative effects on solar cell performances. Martí et al. have reported the effect of strain induced by S-K growth on QDSC. In Fig. 29, (a) shows the sample design of fabricated in their study and (b) shows the internal quantum efficiency (IQE) corresponding to QD-IBSC with 10, 20, and 50 stacked QD layers grown on GaAs substrate. ${ }^{106}$ All the samples are grown by MBE. The multi-stacked InAs/GaAs QDs layers are inserted between $p$-GaAs emitter and $n$-GaAs base layer, and the QDs were formed by S-K growth mode with $\sim 2.7$ monolayers (ML) of InAs coverage. As shown in Fig. 29(b), the extended QE response provided by the InAs QD layers for wavelengths exceeding that of the GaAs absorption edge $\left(\lambda_{\mathrm{GaAs}} \sim 880 \mathrm{~nm}\right)$. Furthermore, the photocurrent production due to the InAs QD layers increases as the number of layers increases from 10 to 20. But, IQE for the QDSC with 50 stacks QD layers decreases considerably from 400 to $880 \mathrm{~nm}$ wavelength region. This result indicates the crystal quality of GaAs host material is degraded by the growth of multi-stacked QDs layers. Figure 30 shows the bright field scanning transmission electron microscopy (STEM) images for the QDSC with 10 and 50 stacked QD (a) $\mathrm{ARC}+\mathrm{Al}_{x} \mathrm{Ga}_{1-x} \mathrm{As}$
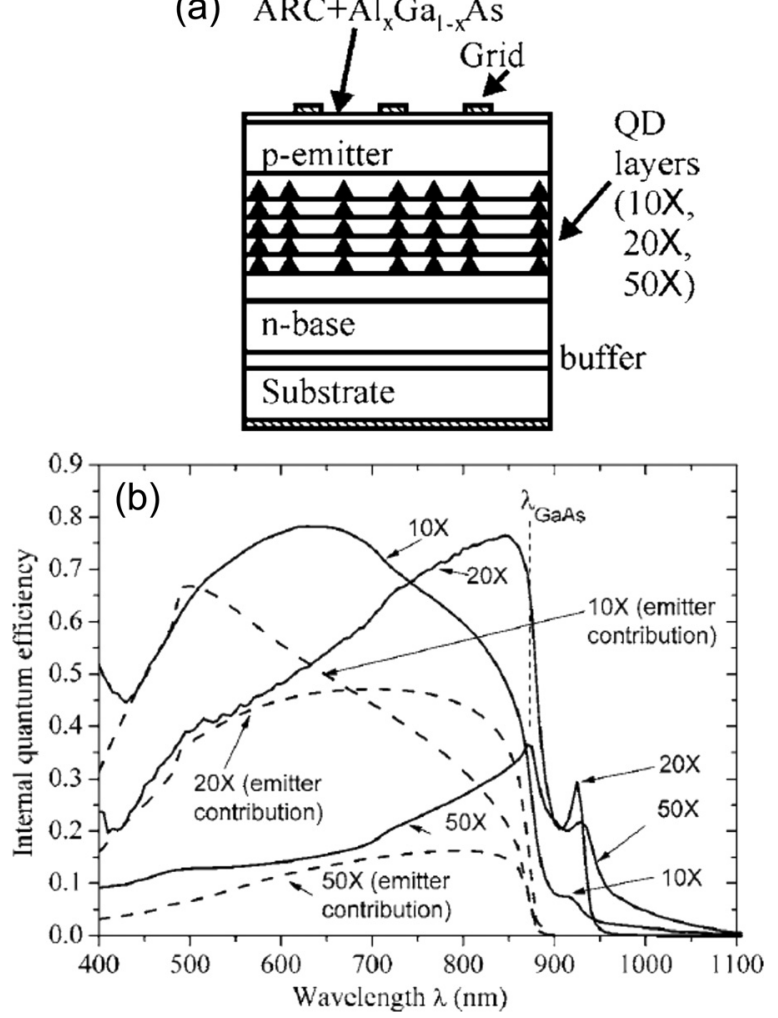

FIG. 29. (a) Simplified internal structure of the IBSCs implemented with QDs and used in these experiments. (b) IQE corresponding to QD-IBSCs with 10, 20, and 50 layers of QD connected lines. The dashed lines correspond to the estimated contribution from the $p$ emitter of the cells. $\lambda_{\mathrm{GaAs}}$ is the GaAs bandgap wavelength and is shown for reference. Reprinted with permission from Martí et al., Appl. Phys. Lett. 90, 233510 (2007). Copyright 2007 AIP Publishing LLC.

layers. The QDs are vertically aligned in the growth direction without misfit dislocations for the QDSC with 10 stacked QD layers. By contrast, a lot of strain-induced dislocations are observed at both the $p$-GaAs emitter and InAs QDs grown regions on sample with 50 stacked QD layers. These defects lead to a significant nonradiative recombination and a reduction in photocurrent productions. The S-K growth in InAs/GaAs system usually results in a degradation
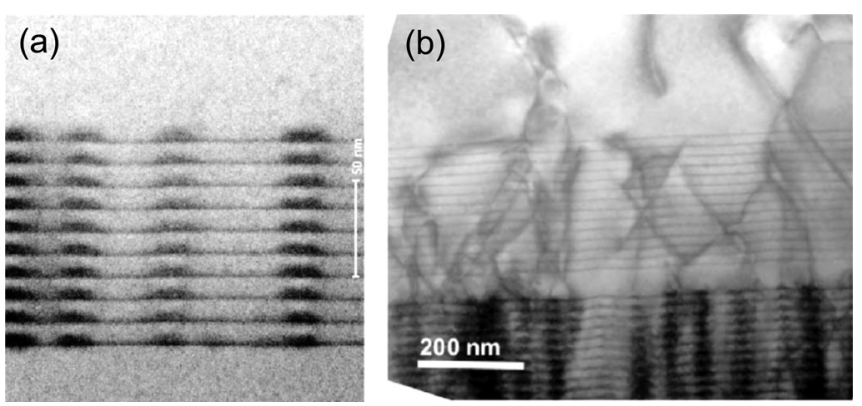

FIG. 30. (a) Bright field STEM image through a 10 stacked QD layers, formed by coverage of $2.7 \mathrm{ML}$ InAs with $10 \mathrm{~nm}$-thick GaAs spacer/capping layers. The $p$-GaAs emitter layer is above the QDs. (b) STEM image of a QD-IBSC with 50 QD layers, formed by coverage of $3.2 \mathrm{ML}$ InAs with $15 \mathrm{~nm}$ GaAs spacer layers. The presence of a high concentration of dislocations in the $p$-emitter and elsewhere is evident. Adapted with permission from Martí et al., Appl. Phys. Lett. 90, 233510 (2007). Copyright 2007 AIP Publishing LLC. 
of stacked QD structure and generation of misfit dislocations, typically after 15-20 layers of stacking, as a result of accumulation of internal strain beyond the critical thickness. Sugaya et al. have reported ultra-high stacked $\mathrm{In}_{0.4} \mathrm{Ga}_{0.6} \mathrm{As} /$ GaAs QDSC fabricated by the intermittent deposition technique. ${ }^{122,123,127}$ The critical thickness in $\mathrm{In}_{0.4} \mathrm{Ga}_{0.6} \mathrm{As} / \mathrm{GaAs}$ system is much thicker than that in InAs/GaAs system. Therefore, $\mathrm{In}_{0.4} \mathrm{Ga}_{0.6}$ As QDs have the advantage of being able to ultra-high stack. The cross-sectional STEM images of 300 stacked $\mathrm{In}_{0.4} \mathrm{Ga}_{0.6} \mathrm{As}$ QD layers are shown in Ref. 127. No dislocations are generated after the stacking up to 300 layers. They have been successful in the stacking of 400 QD layers while keeping the high crystal quality. The thicknesses of the 400 layers stacked $\mathrm{In}_{0.4} \mathrm{Ga}_{0.6} \mathrm{As} / \mathrm{GaAs} \mathrm{QD}$ structure is thought to be still lower than the critical value of misfit dislocation. However, in InGaAs/GaAs system, sufficiently thick GaAs spacer layers are needed to stack the InGaAs QDs. Moreover, the thick wetting layer is formed before the formation of InGaAs QD, because the critical thickness of the growth mode transition from $2 \mathrm{D}$ to $3 \mathrm{D}$ is thicker than that of InAs QD.

On the other hand, tensile-strained barriers have been studied to balance out or compensate for the compressive strain induced in QD active regions. ${ }^{107-114,150,151}$ This growth method is called the strain-compensation or strainbalance technique. Oshima et al. have studied multi-stacked InAs QDs layers on GaAs substrates by using straincompensation, in which GaNAs dilute nitride spacer layers were used as strain compensating layer (SCL). ${ }^{113}$ The conditions for strain compensation are determined by the following equations: ${ }^{152}$

$$
\begin{gathered}
d_{\mathrm{QD}} \cdot \sigma_{\mathrm{QD}}+\sigma_{\mathrm{spacer}} \cdot \varepsilon_{\mathrm{spacer}}=0, \\
\sigma_{\mathrm{QD}}=\left(a_{\mathrm{QD}}-a_{\text {sub }}\right) / a_{\text {sub }}, \\
\sigma_{\text {spacer }}=\left(a_{\text {spacer }}-a_{\text {sub }}\right) / a_{\text {sub }}
\end{gathered}
$$

where $d_{\mathrm{QD}}$ and $d_{\text {spacer }}$ are the deposition thicknesses of the QD layer and the spacer layer; $a_{\mathrm{QD}}, a_{\mathrm{sub}}$, and $a_{\text {spacer }}$ are the lattice constant of QD layer, substrate, and spacer layer; and $\sigma_{\mathrm{QD}}$ and $\sigma_{\text {spacer }}$ are the amounts of strain with respect to the substrate in the QD layer and the spacer layer, respectively. Figure 31(a) shows high resolution X-ray diffraction (XRD) spectra for samples with 20 layers stacked InAs QDs embedded in GaNAs SCL and GaAs spacer layer. ${ }^{109}$ The samples are grown on GaAs (001) substrate. The satellite peaks originating from the periodic structure can be clearly observed in each sample, and hence abrupt heterointerfaces are maintained after stacking. For InAs/GaNAs system, the zerothorder satellite peak $S(0)$ indicated by an arrow in Fig. 31(a) shows a near lattice match with GaAs substrate. Thus, the net average compressive strain induced by InAs QDs layers is approximately compensated by GaNAs SCLs though not precisely balanced out in all three dimensions of QDs.

Figure 31(b) shows the effect of strain-compensation on the IQE spectra of multi-stacked QDSCs. Four kinds of samples were fabricated on GaAs (001) substrate in that work. Two of them are strain-compensated QDSCs with 10 and 20
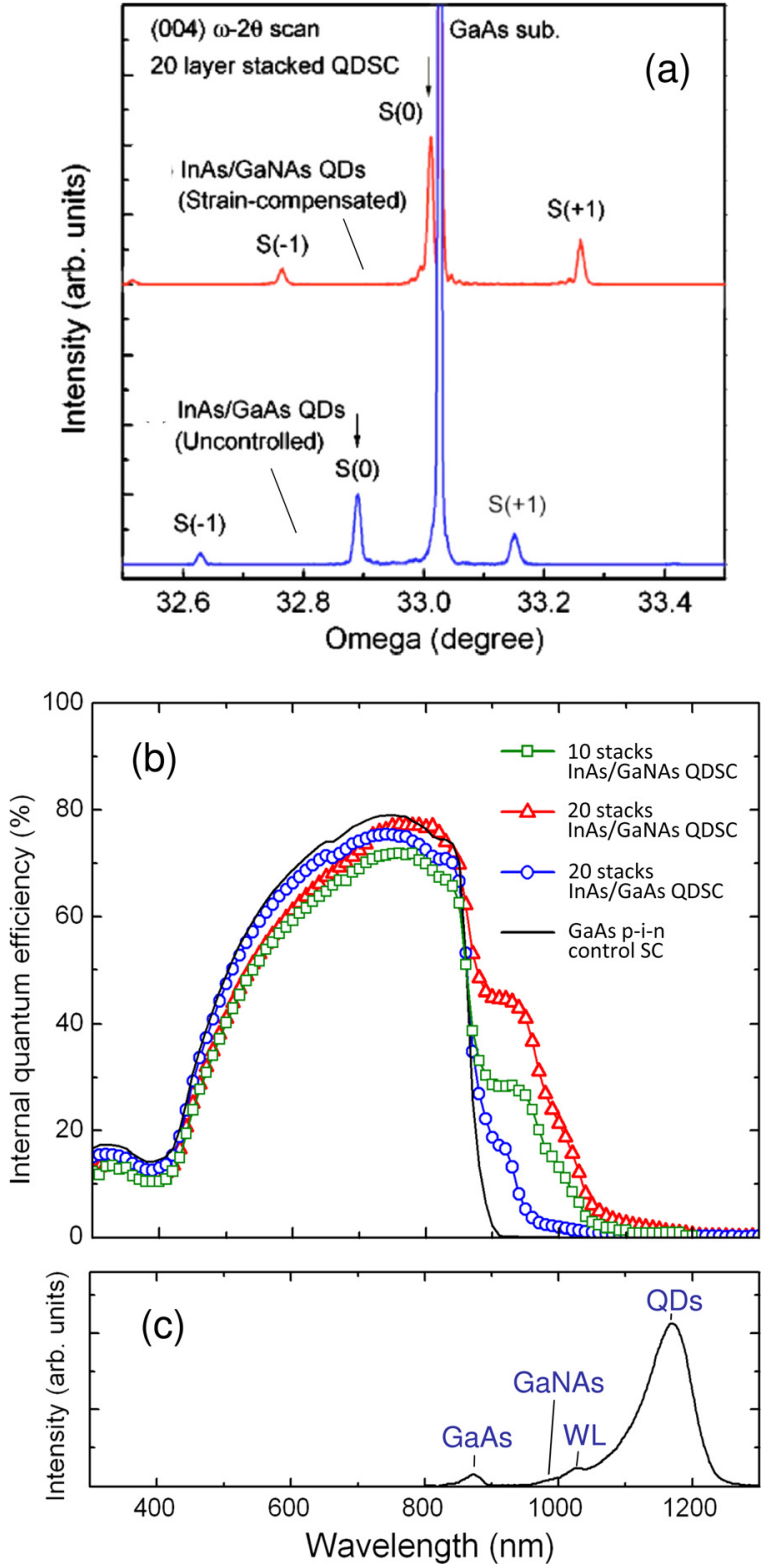

FIG. 31. (a) Symmetric (004) XRD patterns for samples with 20 stacked layers of InAs QDs structure embedded in $20 \mathrm{~nm}$-thick GaNAs SCLs and $20 \mathrm{~nm}$-thick GaAs spacer layers, respectively. $\mathrm{S}( \pm x)$ means $x$ th-order satellite peak. (b) IQE spectra for strain-compensated InAs/GaNAs QDSC with 10 stacks, strain-compensated InAs/GaNAs QDSC with 20 stacks, uncompensated InAs/GaAs QDSC with 20 stacks, and GaAs $p-i-n$ control cell. (c) PL spectrum at RT for 20 layers stacked InAs/GaNAs QDSC. Reprinted with permission from Oshima et al., Appl. Phys. Lett. 93, 083111 (2008). Copyright 2008 AIP Publishing LLC.

stacked pairs of the InAs QDs layer with 2.0 ML coverage and a $20 \mathrm{~nm}$-thick $\mathrm{GaN}_{0.01} \mathrm{As}_{0.99} \mathrm{SCL}$. Another is comprised of 20 pairs of InAs QDs and GaAs spacer layers, and hence the QD structure is strained. The other is a GaAs control cell with a $400 \mathrm{~nm}$-thick $i$-GaAs layer used as a reference. The four samples were identical in the structure except for the $i$-layer region. Additionally, Fig. 31(c) shows the photoluminescence (PL) spectrum for 20 layers stacked InAs/ GaNAs QDSC which is used to identify the photo-absorption edge for each layer. A PL emission peak from the ground state of InAs QDs is observed at around $1170 \mathrm{~nm}$. In addition, a shoulder peak originating from both GaNAs spacer 
layer and InAs wetting layer is seen at around $980 \mathrm{~nm}$ for both InAs/GaNAs strain-compensated QDSCs and $880 \mathrm{~nm}$ for InAs/GaAs uncompensated QDSC, respectively. Second, the absorption edge is redshifted from $1150 \mathrm{~nm}$ for InAs/ GaAs QDSC up to $1200 \mathrm{~nm}$ for InAs/GaNAs QDSC, and this is because the quantum confinement is lower at the $\mathrm{CB}$ interface when GaNAs is used as the barrier instead of GaAs. The filtered $J_{\mathrm{SC}}$ of 20 stacks InAs/GaNAs QDSC beyond the GaAs bandedge, which gives the contribution from QD layer, was determined to be $2.47 \mathrm{~mA} / \mathrm{cm}^{2}$ by taking the product of IQE and AM 1.5 spectrum. This value is twice that of 10 stacks InAs/GaNAs QDSC and four times larger than that of 10 stacks uncompensated QDSC, respectively. The degradation of QEs in the shorter wavelength is not observed after increasing the QD layer from 10 to 20 stacks suggests that the crystalline quality is maintained in the strain-compensated QDSCs as the contribution to the photocurrent generated by the $p$-GaAs emitter is not affected. Though the GaNAs SCL increases photocurrent, it causes a substantial drop in $V_{\mathrm{OC}}$ due to an increase in the $\mathrm{CB}$ offset at the GaAs/GaNAs heterointerfaces at both ends of the $i$-layer, which in turn increases the recombination loss within the $i$-layer thereby leading to increasing dark current. ${ }^{12}$

Hubbard et al. have fabricated strain-compensated QDSC using GaP SCL as illustrated in Fig. 32(a). ${ }^{108}$ In order to investigate the effect of strain-compensation, 5 stacks InAs QDSC with and without GaP SCL were grown by MOVPE. For the strain-compensated QDSC, each InAs QD layer is followed by the $6 \mathrm{~nm}$-thick GaAs, a $1.4 \mathrm{~nm}$-thick $\mathrm{GaP}$, and a $4 \mathrm{~nm}$-thick GaAs. The QDSC without straincompensation consists of 5 stacked pairs of the InAs QD and $10 \mathrm{~nm}$-thick GaAs. In addition, the control GaAs cell with $100 \mathrm{~nm}$-thick $i$-region is fabricated as a reference. Figure 32(b) shows $J-V$ curves under 1 sun of AM 0 solar spectrum for both a 5 layers stacked InAs QDSC with and without strain-compensation and for the GaAs control cell. The QDSC without strain-compensation exhibits substantial degradation in overall cell performance compared with GaAs control cell. Degradation in both $J_{\mathrm{SC}}$ and $V_{\mathrm{OC}}$ is likely due to increased minority carrier recombination by the inserting QD layers. The reduced $F F$ results from a decrease in majority carrier mobility in the $p$-GaAs emitter region. Mobility degradation has been shown to be related to misfit dislocations which may originate at the QD layer as stated above. The $V_{\mathrm{OC}}$ of $0.83 \mathrm{~V}$ in the QDSC with strain-compensation is significantly higher than that of QDSC without straincompensation. Further, the $J_{\mathrm{SC}}$ also improved to the extent that there is a slight increase compared to the GaAs control cell. The increased $J_{\mathrm{SC}}$ of the device resulted by improving the crystal quality in the $p$-GaAs emitter region and from the photo-generated current contributed by the InAs QDs. The improvement in $F F$ can be attributed to improved carrier transport through the intrinsic and emitter regions. The $V_{\mathrm{OC}}$ is also improved by strain-compensation, but the value is about $190 \mathrm{meV}$ lower than that of the GaAs control cell.

Bailey et al. have fabricated the strain-compensated QDSC with high $V_{\mathrm{OC}}$ by reducing the InAs coverage. ${ }^{125}$ Figure 33 shows the $J-V$ curve under 1 sun of AM 0 solar spectrum measured for the GaAs control cell and the 10 layers
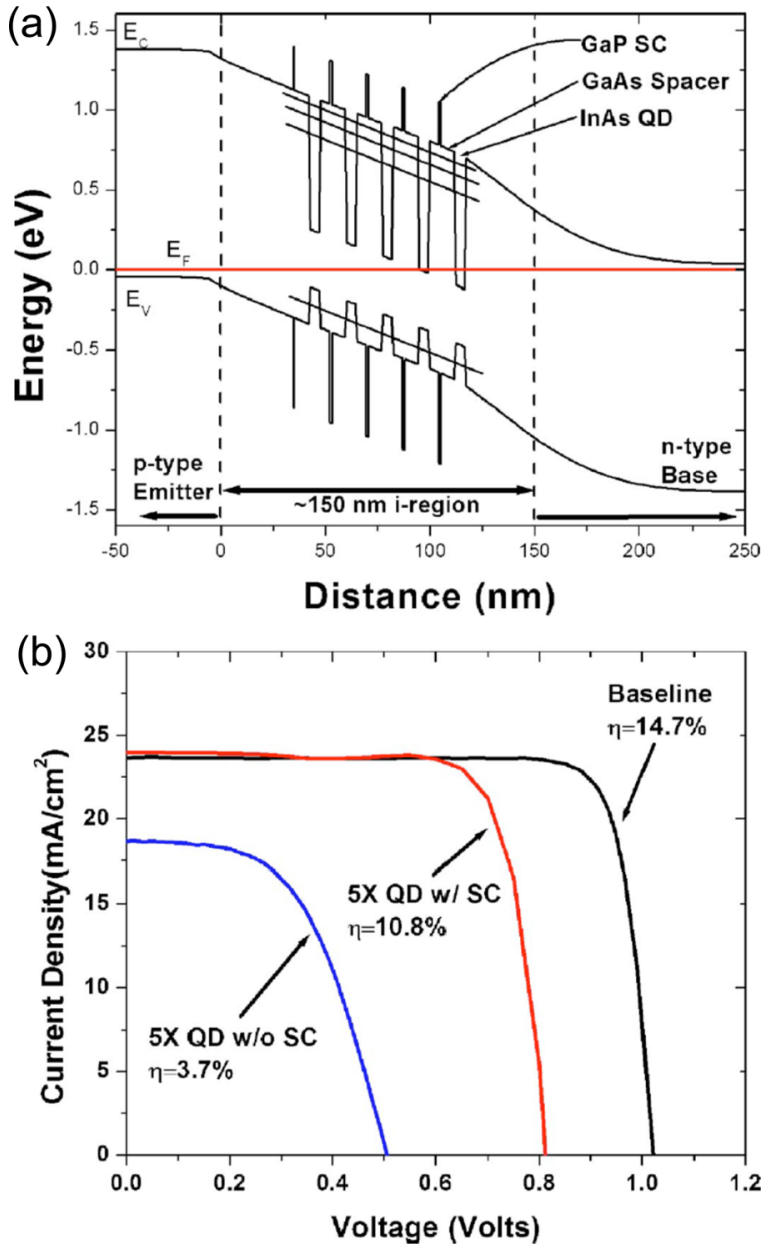

FIG. 32. (a) Simplified energy band diagram illustrating the design of the strain-compensated QDSC. The diagram is generated near the $i$-region of a $p-i-n$ cell containing InAs QDs with GaP SCL. (b) $J-V$ curves under 1 sun of AM 0 solar spectrum for the $p-i-n$ GaAs control cell and QDSCs both with and without strain-compensation. 5 layers stacked InAs QDs are inserted into the intrinsic layer for all QDSCs. Reprinted with permission from Hubbard et al., Appl. Phys. Lett. 92, 123512 (2008). Copyright 2008 AIP Publishing LLC.

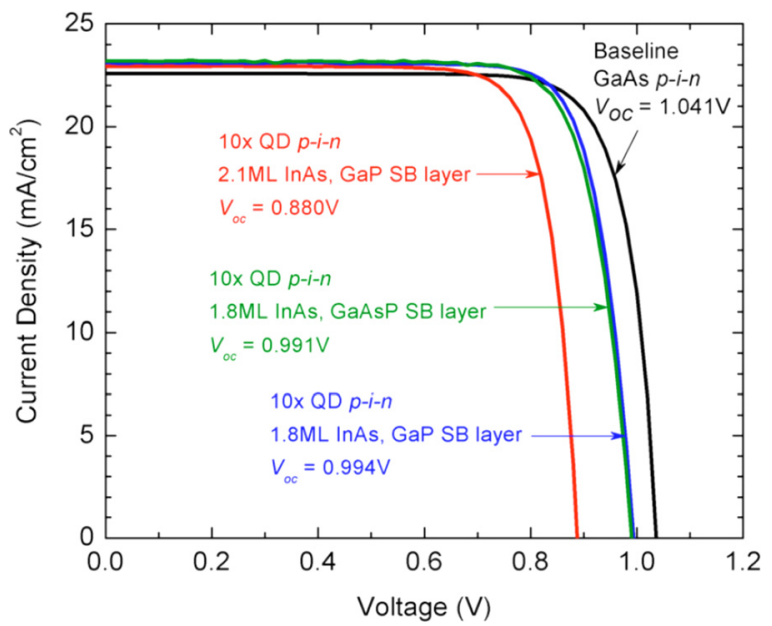

FIG. 33. $J-V$ curves under 1 sun of AM 0 solar spectrum for GaAs control cell and QDSCs with 2.1 ML InAs and GaP strain-balanced (SB) layer and 1.8 ML InAs coverage with both $\mathrm{GaP}$ and GaAsP SB layer. 10 layers stacked InAs QDs are inserted into the intrinsic layer for all QDSCs. Reprinted with permission from Bailey et al., Appl. Phys. Lett. 98, 163105 (2011). Copyright 2011 AIP Publishing LLC. 
stacked strain-compensation QDSC with 2.1 ML and 1.8 ML InAs coverage. The condition of strain-compensation is determined by continuum elasticity theory derived based on QD parameter such as height and in-plane density. ${ }^{153}$ The $V_{\mathrm{OC}}$ of QDSC with 2.1 ML InAs coverage and GaP SCL is about $160 \mathrm{mV}$ lower than that of GaAs control cell as is the case with Fig. 32(b). QDSC with 2.1 ML InAs coverage has the giant QDs caused by the island coalescence and bimodal size distribution according to their study. ${ }^{129,154}$ As the result, the dark current increases due to the localized strain induced by the larger QD. QDSC with 1.8 ML InAs coverage and GaP SCL exhibits a significant improvement in the $V_{\mathrm{OC}}$. The $V_{\mathrm{OC}}$ value is $0.994 \mathrm{~V}$ for the $1.8 \mathrm{ML}$ InAs QDSC using GaP SCL, and the voltage loss compared to GaAs control cell is only $47 \mathrm{mV}$. This is because the nonradiative recombination is reduced by decreasing the density of larger defective QDs. In exactly the same manner, they have succeeded in the development in QDSC with 40 layers stacked QDs while maintaining the high $V_{\mathrm{OC}}{ }^{129}$ The conversion efficiency reaches $14.3 \%$ under irradiation of AM 0 solar spectrum. Recently, strain-compensation growth techniques have shown a significantly improved multi-stacked QD quality as well as characteristics of QDSCs even after stacking of 50 QD layers by S-K growth on GaAs substrate. ${ }^{113,114}$ On InP substrate, high material quality has been reported in 300 layers stacked InAs QDs using AlGaInAs strain-compensation layer. ${ }^{152}$

\section{Characteristics of type-II QD structures}

The photocurrent generation by TSPA is the central operating principle of IBSC. In order to further increase the conversion efficiency, the enhancement of TSPA rate via IB is a key issue. The type-II energy band alignment is one of the effective measures for increasing the optical transition from IB to $\mathrm{CB}$, since the structure lead to longer carrier lifetimes. ${ }^{155,156}$ The InAs/GaAs system has been vigorously studied in QD growth. This material combination has a type-I energy band alignment with a radiative recombination lifetime of typically few ns. ${ }^{157,158}$ This is due to the fact that both the electrons and holes are confined in the QD layer. However, adding $\mathrm{Sb}$ into GaAs barrier layers reduces the VB offset energy between QD and barrier layers, and increasing $\mathrm{Sb}$ composition in GaAsSb alloy eventually changes the band alignment to type-II. ${ }^{159,160}$ As a result, the radiative recombination lifetime becomes significantly longer than that for type-I structure. For this, QDSCs with type-II band alignment have received attention in recent years. ${ }^{132-136,138-140}$

Figure 34(a) shows the PL spectra for the InAs QDs embedded by $4.5 \mathrm{~nm}$-thick $\mathrm{GaAs}_{1-x} \mathrm{Sb}_{x}$ cap layer with different $\mathrm{Sb}$ compositions measured by Chang et al. ${ }^{161}$ The measurement is carried out at $12 \mathrm{~K}$. A redshift in the PL peak energy is observed with increasing $\mathrm{Sb}$ composition, which is caused by the change of VB offset in the GaAsSb cap layer. Figure 34(b) indicates the excitation power dependent PL spectra for the InAs QDs embedded by $\mathrm{GaAs}_{0.84} \mathrm{Sb}_{0.16}$ layer. The peak energy of InAs QD PL emission exhibits a blueshift with increasing the cube root of excitation power $P_{\text {ex }}$ as shown in Fig. 34(c). Chiu et al. explained that the blueshift is due to the band bending induced by the spatially separated
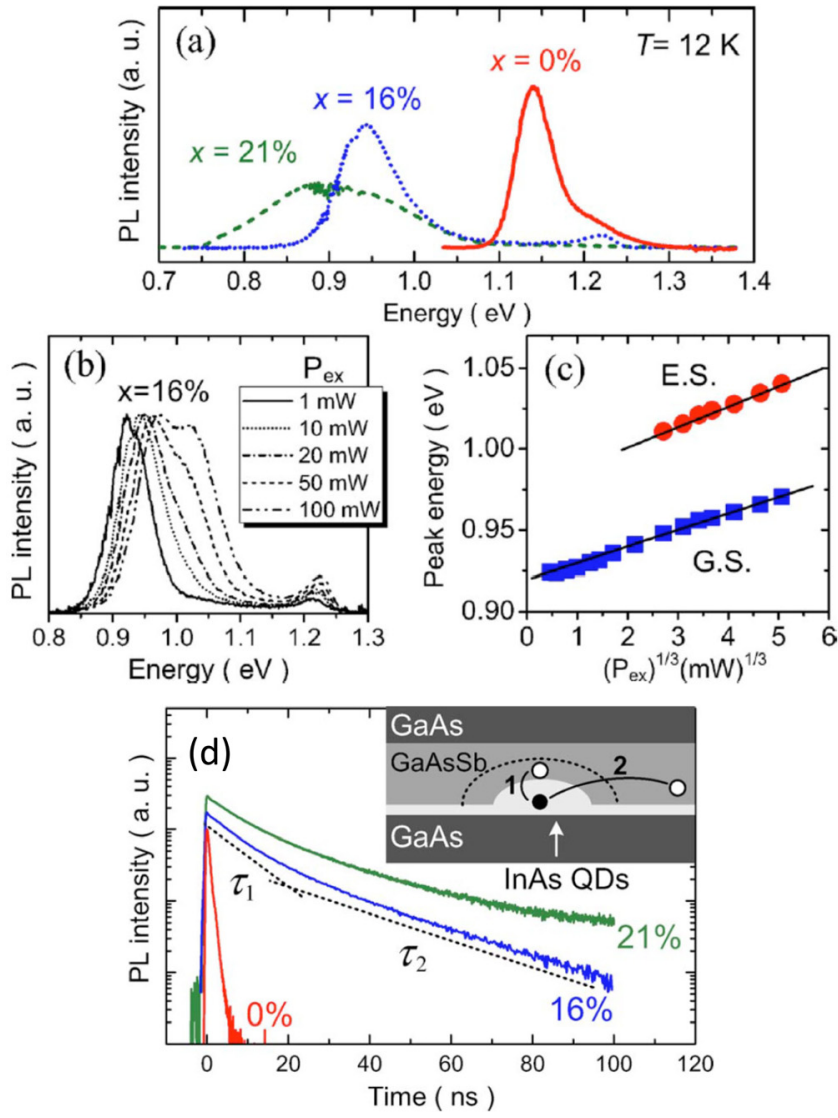

FIG. 34. (a) PL spectra of the $\mathrm{GaAs}_{1-x} \mathrm{Sb}_{x}$ covered InAs QDs with different $x$. (b) Power dependent PL spectra of the InAs QDs covered by $\mathrm{GaAs}_{0.84} \mathrm{Sb}_{0.16}$. (c) The Ground State (G.S.) and the Excited State (E.S.) peak energies as a function of $P_{e x}^{1 / 3}$ for the InAs QDs covered by $\mathrm{GaAs}_{0.84} \mathrm{Sb}_{0.16}$. (d) TR-PL decay traces for the investigated QD samples with different $\mathrm{Sb}$ composition. The inset depicts the sample structure and the underlying recombination processes responsible for the faster one and slower two decay components. Reprinted with permission from Chang et al., Appl. Phys. Lett. 93, 033107 (2008). Copyright 2008 AIP Publishing LLC.

photo-carriers in a type-II band structure. ${ }^{162}$ The strong localized carriers around the interface of type-II band structure form a charged plane, and hence produce an approximated triangular potential well. The electric field strength of the interfaces is then written by following equation:

$$
\varepsilon=\frac{2 \pi e n_{\mathrm{w}}}{\varepsilon_{0}} \propto P^{1 / 2},
$$

where $n_{\mathrm{w}}$ is the electron density and $P$ is the excitation photon flux. The ground electron state $E_{\mathrm{gs}}$ in a triangular potential well is given by

$$
E_{\mathrm{gs}}=\text { const } \cdot \varepsilon^{2 / 3} \propto P^{1 / 3} .
$$

The quantum state is thus expected to increase proportionally with the cube root of the excitation power. Accordingly, the result obtained in Fig. 34(c) indicates that type-II structure is indeed formed between InAs QDs and GaAsSb layers.

Figure 34(d) shows the time-resolved PL (TR-PL) of the InAs/ $\mathrm{GaAs}_{1-x} \mathrm{Sb}_{x}$ QDs measured at $12 \mathrm{~K}$. The decay time for the InAs/GaAs QDs $(x=0 \%)$ with type-I band alignment is $\tau \approx 0.8 \pm 0.2 \mathrm{~ns}$. In contrast, InAs/GaAsSb QDs exhibit much longer decay times than that of InAs/GaAs QDs. The 
TR-PL decay profile of InAs/GaAsSb QDs with type-II band alignment has double components of a faster initial part and a slower tail part. They can be fitted by a double exponential function

$$
I(t)=A_{1} \exp \left(-t / \tau_{1}\right)+A_{2} \exp \left(-t / \tau_{2}\right) .
$$

For the InAs/ $\mathrm{GaAs}_{0.84} \mathrm{Sb}_{0.16} \mathrm{QDs}$, the evaluated PL decay time is $\tau_{1}=7.5 \mathrm{~ns}$ and $\tau_{2}=24 \mathrm{~ns}$, with a relative ratio of $A_{2} / A_{1}=0.4$. On the other hand, the decay time for the InAs/ $\mathrm{GaAs}_{0.79} \mathrm{Sb}_{0.21}$ QDs is $\tau_{1}=8.2 \mathrm{~ns}$ and $\tau_{2}=29 \mathrm{~ns}$, with a more pronounced slower decay component $A_{2} / A_{1}=0.5$.

Nishikawa et al. have investigated the relationship between carrier lifetime and VB offset in the InAs/GaAsSb system. ${ }^{163}$ Figure 35 shows the dependence of radiative recombination time $\left(\tau_{\text {rad }}\right)$ on $\mathrm{VB}$ offset $(\Delta \mathrm{VBM})$ between InAs QD and GaAsSb layer. $\triangle \mathrm{VBM}=-65,-23$, and $27 \mathrm{meV}$ correspond to the $\mathrm{Sb}$ compositions of $18 \%, 14 \%$, and $10 \%$, respectively. The lifetime is normalized by the value for InAs/GaAs type-I QD system. The $\tau_{\text {rad }}$ increases with increasing $\mathrm{Sb}$ composition. This is due to the fact that the penetration of hole wavefunction into the QDs becomes smaller for sample with higher Sb composition. Further, they have achieved the QD structure with extremely long carrier lifetime by inserting a thin GaAs wall layer between InAs QD and GaAsSb layers. The details of sample structure are shown in Ref. 164.

Nishikawa et al. have also demonstrated the QD structure with extremely long carrier lifetimes by inserting thin GaAs wall layers between InAs QD and GaAsSb layers as show in Fig. 36. ${ }^{164}$ Figure 36(a) shows the PL decay profiles for the three samples: InAs/GaAs type-I QDs, InAs/ $\mathrm{GaAs}_{0.82} \mathrm{Sb}_{0.18}$ type-II QDs, and InAs/GaAs/ $\mathrm{GaAs}_{0.82} \mathrm{Sb}_{0.18}$ type-II QDs measured at $4 \mathrm{~K}$. The PL decay time of InAs/ $\mathrm{GaAs} / \mathrm{GaAs}_{0.82} \mathrm{Sb}_{0.18}$ QDs is much longer than those of InAs/GaAs and InAs/GaAs ${ }_{0.82} \mathrm{Sb}_{0.18}$ QDs. Because the carrier lifetime of these samples changes with the delay time associated with reduction of the number of carriers by

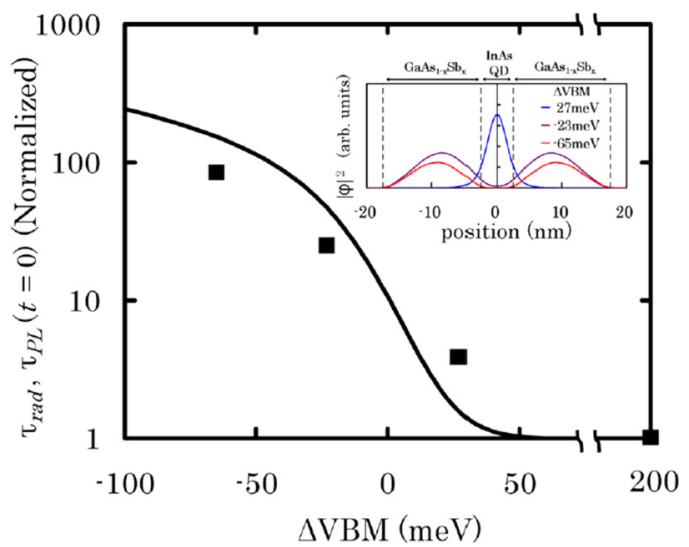

FIG. 35. Comparison for calculated $\tau_{\text {rad }}$ (solid line) vs. experimentally determined PL decay time $\tau_{P L}(t=0)$ (squares) at $77 \mathrm{~K}$ under weak excitation of $38 \mathrm{~mW} / \mathrm{cm}^{2} . \Delta \mathrm{VBM}=-65,-23$, and $27 \mathrm{meV}$ correspond to the $\mathrm{Sb}$ compositions of $18 \%, 14 \%$, and $10 \%$, respectively. The lifetime is normalized by the value for the sample without $\mathrm{Sb}$. Inset illustrates the magnitude of hole existence probability. Reprinted with permission from Nishikawa et al., J. Appl. Phys. 111, 044325 (2012). Copyright 2012 AIP Publishing LLC.

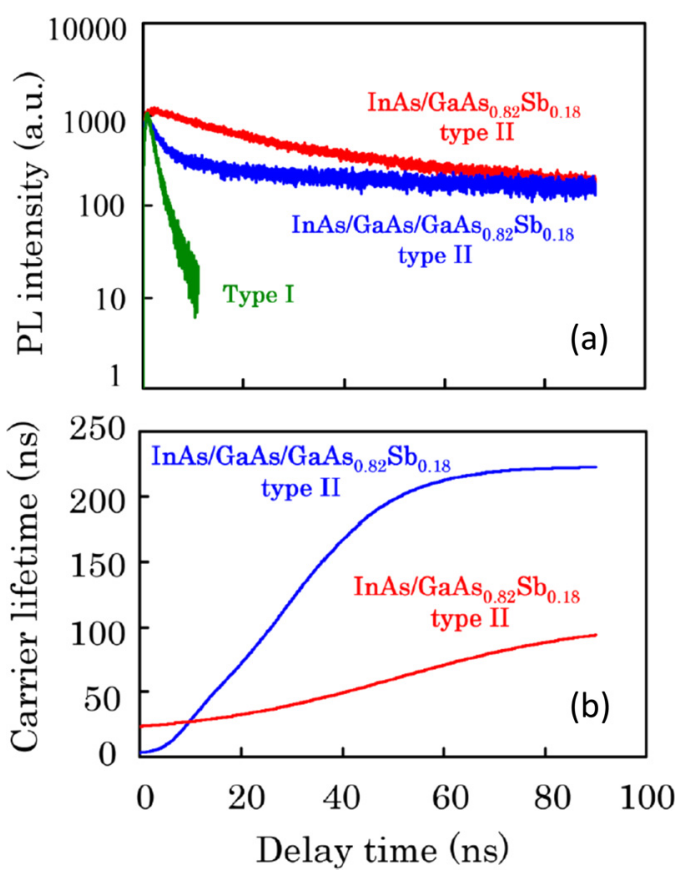

FIG. 36. (a) PL decay profiles for the three samples, InAs/GaAs type-I QDs, InAs/ $\mathrm{GaAs}_{0.82} \mathrm{Sb}_{0.18}$ type-II QDs, and InAs/GaAs/GaAs $0 .{ }_{02} \mathrm{Sb}_{0.18}$ type-II QDs. (b) Time evolution of carrier lifetime for the two samples with type-II band structures. Measurement temperature is $4 \mathrm{~K}$. Excitation intensity is 38 $\mathrm{mW} / \mathrm{cm}^{2}$. Reprinted with permission from Nishikawa et al., Appl. Phys. Lett. 100, 113105 (2012). Copyright 2012 AIP Publishing LLC.

recombination, they derived the time dependent carrier lifetime $\tau$ by following equation:

$$
\tau(t)=\frac{-I(t)}{\frac{\partial I(t)}{\partial t}} .
$$

Figure 36(b) shows the time evolution of $\tau$ of the InAs/ $\mathrm{GaAs}_{0.82} \mathrm{Sb}_{0.18}$ and InAs/GaAs/GaAs ${ }_{0.82} \mathrm{Sb}_{0.18}$ type-II QDs. The long carrier lifetime over $200 \mathrm{~ns}$ is observed for the GaAs wall inserted InAs/GaAs/ $\mathrm{GaAs}_{0.82} \mathrm{Sb}_{0.18}$ type-II QDs. The value is more than 200 times longer than that of InAs/ GaAs type-I QDs. In the InAs/GaAs/GaAs ${ }_{0.82} \mathrm{Sb}_{0.18}$ type-II QD structure, the holes in the InAs QDs immediately escape into the GaAsSb layer through the $2 \mathrm{~nm}$-thick GaAs wall layer by tunneling in the order of $10 \mathrm{ps}$. Consequently, the extremely long carrier lifetime is achieved because the electrons confined in InAs QDs and the holes in the GaAsSb layers are separated before the recombination. This long carrier lifetimes in QD layer are favorable in order to increase the optical generation rate and hence photocurrent production from IB to CB on QD-IBSC.

Shoji et al. have investigated the effect of type-II band alignment on TSPA of QDSC. The samples are fabricated three different types of QD-IBSCs on GaAs (311)B substrate by MBE. ${ }^{128}$ InGaAs QDs grown on a high-index GaAs (311)B substrate exhibit superior in-plane spatial ordering characteristics. ${ }^{144}$ Figure 37 (a) shows the schematic structure of $p-i-n$ QDSC in that work. The 8 stacked pairs of $\mathrm{In}_{0.4} \mathrm{Ga}_{0.6} \mathrm{As} \mathrm{QDs} / \mathrm{GaAs}$ or GaAsSb spacer layer as shown in Figs. 37(b)-37(d) are inserted into the $i$-layer. The $i$-layer is grown at $480^{\circ} \mathrm{C}$, and the growth rates of QDs, GaAs, and 
(a) Cell structure

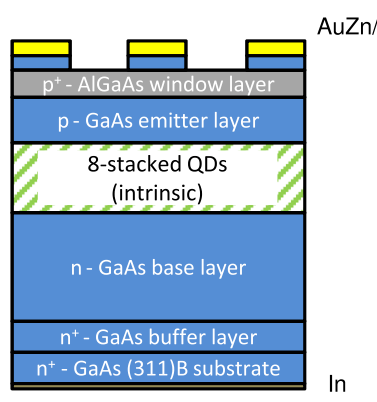

(b) Buffer layer: GaAs Cap layer: GaAs

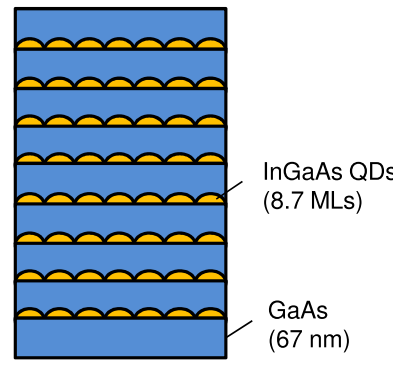

(c) Buffer layer: GaAs Cap layer: GaAsSb

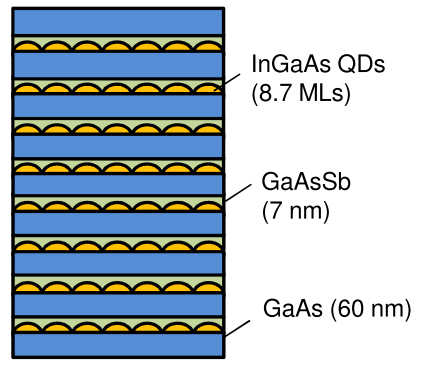

(d) Buffer layer: GaAsSb Cap layer: GaAsSb

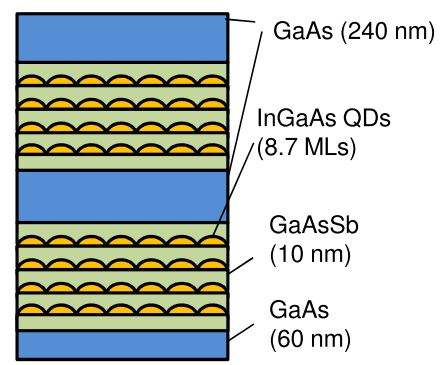

FIG. 37. (a) Schematic structure of fabricated $p-i-n$ QDSC. (b)-(d) Three types of 8 layers stacked QDs structures inserted into the intrinsic region of (a) in order to investigate the effect of band alignment on TSPA of QDSC. The material of both buffer and cap layers of InGaAs QDs are changed in each sample.

GaAsSb layers were $1.0 \mu \mathrm{m} / \mathrm{h}, 0.6 \mu \mathrm{m} / \mathrm{h}$, and $0.6 \mu \mathrm{m} / \mathrm{h}$, respectively. The $\mathrm{In}_{0.4} \mathrm{Ga}_{0.6} \mathrm{As} / \mathrm{GaAs}$ QDSC has type-I band alignments, while both QDSCs with QDs capped and sandwiched by GaAsSb have type-II band alignments in the heteroepitaxial interface between QD and GaAsSb layers. In order to detect the production of a photocurrent as a direct result of optical transition of electrons from IB to $\mathrm{CB}$, an IR light source was added to the standard $\mathrm{EQE}$ measurement system. ${ }^{124}$ The IR light source is constituted by a Xenon lamp with an AM 1.5 filter and IR pass filter that altogether allow only the wavelength region of $\lambda>1400 \mathrm{~nm}$. The illumination from this IR source can only pump electrons from IB to $\mathrm{CB}$, but do not have sufficient energies to pump electrons from VB to IB or directly from VB to $\mathrm{CB}$. The EQE spectra are then measured, with monochromatic light, first without and then with a continuous illumination of IR light. The details of measurement system are given in Sec. III D 2 . Figure 38 shows the $\triangle \mathrm{EQE}$, taken with the difference of EQE with and without IR illumination measured at RT. The scan range is from 920 to $1050 \mathrm{~nm}$ in wavelength, in order to estimate the contribution of absorption via IB. For all samples, a production of photocurrent due to absorption of IR photons producing IB to $\mathrm{CB}$ optical transition is clearly observed at RT. In addition, both samples with GaAsSb layers show a higher $\triangle \mathrm{EQE}$ response than that for InGaAs/ GaAs structure. Especially, the production of TSPA for closely stacked QDs sandwiched in between GaAsSb layers on both sides increases compared to QDs with one-sided GaAsSb layer. These results are attributed to an increased carrier lifetime induced by spatially separated electrons and holes on the type-II band structure.

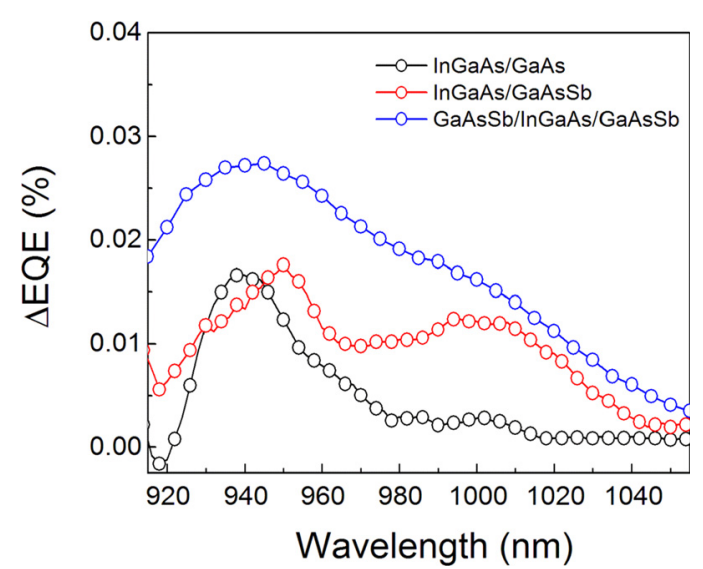

FIG. 38. Difference in EQE values, $\triangle \mathrm{EQE}$, with $\left(\mathrm{EQE}_{w / \mathrm{IR}}\right)$ and without $\left(\mathrm{EQE}_{w / o} \mathrm{IR}\right)$ IR light illumination.

\section{Effect of doping on QDSCs}

The doping of impurities into such InAs QDs is an efficient approach to improve the optical property of the device such as lasers, optical amplifiers, and mid-IR sensors. ${ }^{165,166}$ The excess carriers provided by the dopant can compensate the carrier loss during device operation and boost intersubband transition. There are basically two techniques to insert the dopants into the QD. One is so-called modulation doping technique where the dopants are inserted into the barrier region prior to the QD growth. ${ }^{167}$ The crystal quality of the QDs is not affected by doping but the doping concentration in each QD is difficult to control by using this technique. The other doping method is direct doping where the dopants are added directly into the QD during the QD growth. It has been reported that crystal defects caused by direct doping deteriorate both the crystal quality and the optical properties of QDs. ${ }^{168,169}$ But recently, Kita et al. have managed to maintain high crystal quality of QD during direct doping by well tuning the growth parameters during the QD assembly stages. $^{31,170}$

The doping into InAs QD has also been applied to fill the IB in InAs QD-IBSC in order to maximize the photocurrent generation by TSPA. IB must be partially filled by means of doping within a reasonable optical length in order to achieve high efficiency. ${ }^{27,88,105,171}$ Yoshida et al. have also recently reported similar calculated results based on self-consistent device simulation method. In the case of doped IB, the generation rate drastically drops near the top emitter layer and the bottom base layer under 1 sun illumination. As illumination gets stronger, such drops in the net generation rates disappear and the position dependence of the net generation rate becomes similar to that with undoped IB under 1000 suns illumination. ${ }^{21}$

In experiment, doping effect on optical properties of InAs/GaAs QD has been widely investigated over the last decades. However, there are limited reports on the experimental study of doping effect on the performance of InAs/ GaAs QDSC.

Martí et al. have used a modulation doping technique to fabricate a QD-IBSC, in which GaAs barrier is delta $(\delta)$ doped with $\mathrm{Si}$ with an areal density approximately equal to 
the QD density $\left(4 \times 10^{10} \mathrm{~cm}^{-2}\right)$ and was inserted between each QD layer, (see Fig. 39(a)). Due to spin degeneration, this condition equals half the density of electronic states in IB. ${ }^{104,172}$ From the $J-V$ characteristics (Fig. 39(b)), it is clear that the inclusion of the QDs degrades the performance of the devices especially the $V_{\mathrm{OC}}$. However, it also clearly shows that the $V_{\mathrm{OC}}$ is dramatically recovered after the QD was $\delta$-doped with Si.

InAs QD based IBSC with direct doping was reported by Okada et al. InAs QD layers were directly doped with $\mathrm{Si}$ impurity during the self-assembling stage of growth, which was found to be the optimal timing for $\mathrm{Si}$ atoms to be doped evenly into QDs. ${ }^{124}$ Inoue et al. showed that the electrical conductivity of a direct Si-doped QD becomes higher than the wetting layer by using conductive atomic force microscopy and that the carriers are preferentially incorporated into QDs. ${ }^{31}$ The sheet density of Si-doping was set to be $5 \times 10^{10} \mathrm{~cm}^{-2}$ per QD layer in order to dope approximately one $\mathrm{Si}$ atom per QD. Figure 40(a) shows the PL spectra measured at $77 \mathrm{~K}$ for 25 layers-stacked InAs/GaNAs straincompensated QDSCs with (i) direct Si-doped and (ii) undoped QDs, respectively. The QDSC with direct Si-doped QDs (i) shows a stronger PL intensity than for undoped QDs (ii) by a factor of 1.7. The results indicate that defect states such as electron traps at the heterointerface are inactivated
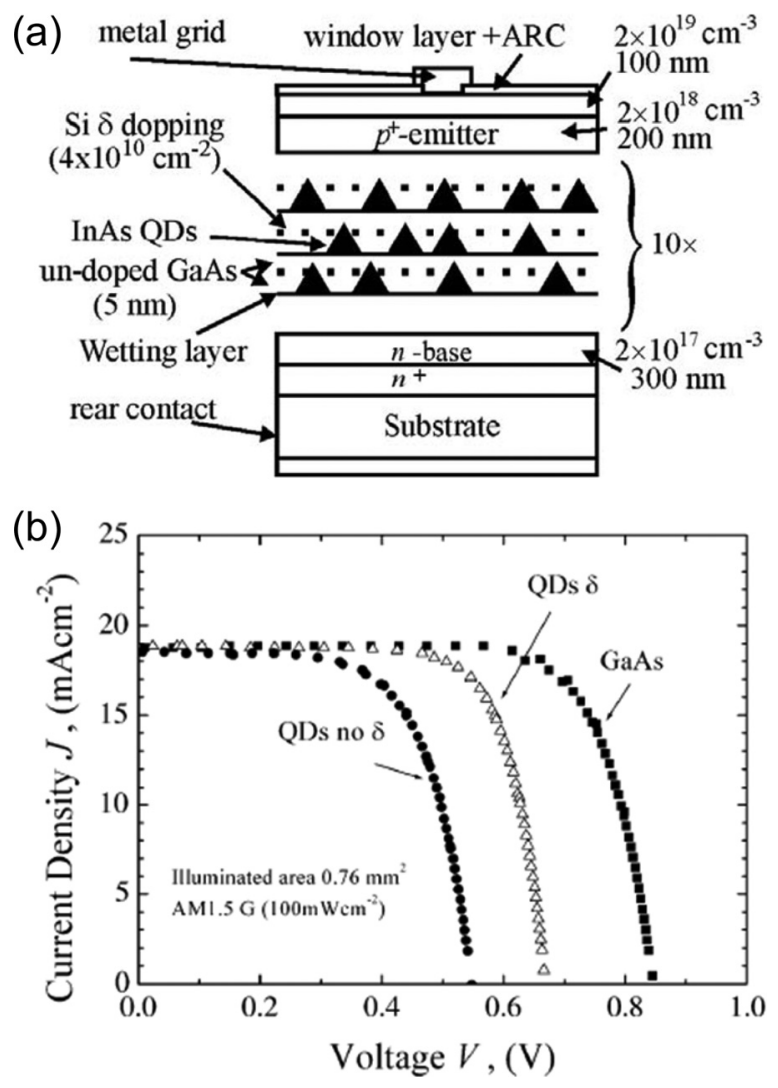

FIG. 39. (a) Layer structure of the QD-IBSCs. (b) $J-V$ curves under illumination. Current density is referred to the illuminated area. For the GaAs cell, $J_{\mathrm{SC}}=18.8 \mathrm{~mA} \mathrm{~cm}^{-2}, V_{\mathrm{OC}}=0.846 \mathrm{~V}$, and efficiency of $12.1 \%$. For the QDs$\delta$ cell, $J_{\mathrm{SC}}=18.8 \mathrm{~mA} \mathrm{~cm}{ }^{-2}, V_{\mathrm{OC}}=0.667 \mathrm{~V}$, and efficiency of $9.3 \%$. For the QD no $\delta$ cell, $J_{\mathrm{SC}}=18.5 \mathrm{~mA} \mathrm{~cm} \mathrm{~cm}^{-2}, V_{\mathrm{OC}}=0.548 \mathrm{~V}$. Reprinted with permission from Martí et al., Thin Solid Films 511-512, 638 (2006). Copyright 2006 Elsevier B.V. (a)
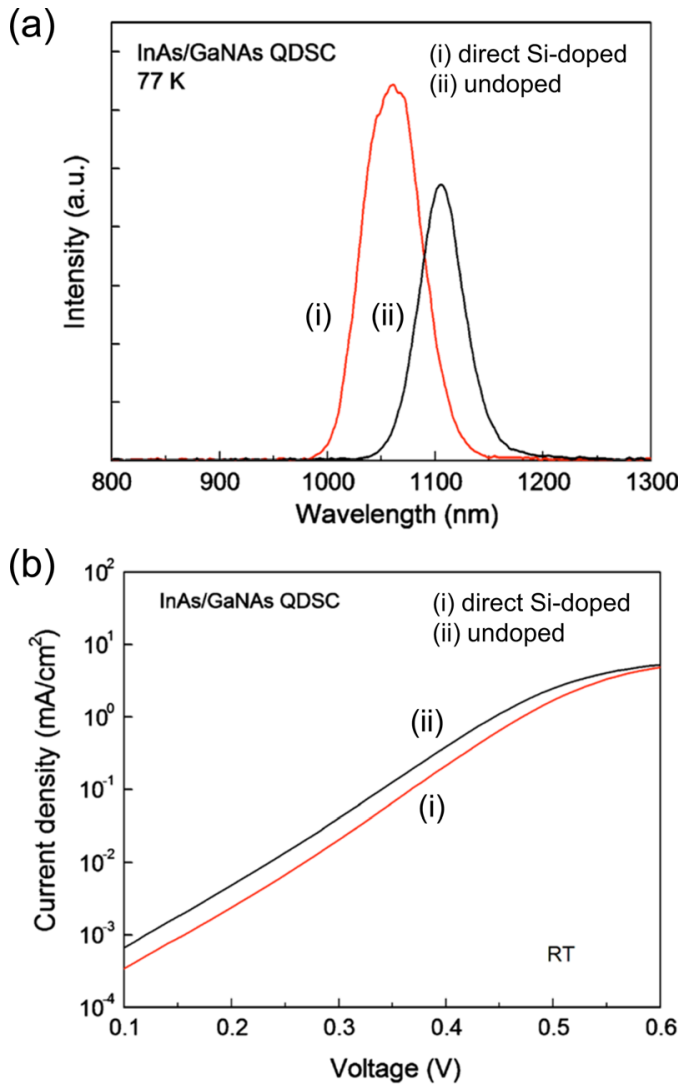

FIG. 40. (a) PL spectra measured for 25 layers-stacked InAs/GaNAs straincompensated QDSCs with (i) direct Si-doped and (ii) undoped QDs, respectively. The measurement temperature was $77 \mathrm{~K}$ and $532 \mathrm{~nm}$ diode-pumped solid-state laser at an output power of $1 \mathrm{~W} / \mathrm{cm}^{2}$ was used as the excitation source. (b) Dark $J-V$ characteristics measured at RT for QDSCs with (i) direct Si-doped and (ii) undoped InAs QDs, respectively. Reprinted with permission from Okada et al., J. Appl. Phys. 109, 024301 (2011). Copyright 2011 AIP Publishing LLC.

by the doped electrons and hence nonradiative recombination loss from CB to VB is reduced. In fact, the dark current measured for direct Si-doped QDSC is roughly halved compared to undoped QDSC as can be seen in Fig. 40(b). The results agree well with PL results leading to the conclusion that the relaxation of electrons by nonradiative process in the QD region is effectively reduced by Si-doping. This result is also consistent with the results shown in Fig. 39(b) where recovery of $V_{\mathrm{OC}}$ was found in the $\delta$-doped sample.

Sablon et al. fabricated a Si-doped InAs QDSC where a $\delta$-doped QD structure contains 20 stacks of QD layers separated by GaAs. ${ }^{173}$ The plane of dopants is placed in the middle of each GaAs layer that separates the QD layers. The dopant sheet densities are $0,2.4 \times 10^{10} \mathrm{~cm}^{-2}, 3.6 \times 10^{10} \mathrm{~cm}^{-2}$, $4.8 \times 10^{10} \mathrm{~cm}^{-2}$, and $7.2 \times 10^{10} \mathrm{~cm}^{-2}$ for providing $0,2,3,4$, and 6 electrons per QD. $J-V$ curves are shown in Fig. 41(a). While the $p$-doped device with the doping density of $4.8 \times 10^{10} \mathrm{~cm}^{-2}$ shows degradation in $J_{\mathrm{SC}}$ compared with the undoped device, the $n$-doped devices show a monotonic increase in $J_{\mathrm{SC}}$ with increasing doping levels. The enhancement in $J_{\mathrm{SC}}$ can be explained either as a consequence via an enhanced TSPA process induced by $n$-doping with IR transition of an electron from the localized to the conducting state, or another doping induced process, where the radiation excites 
(a)
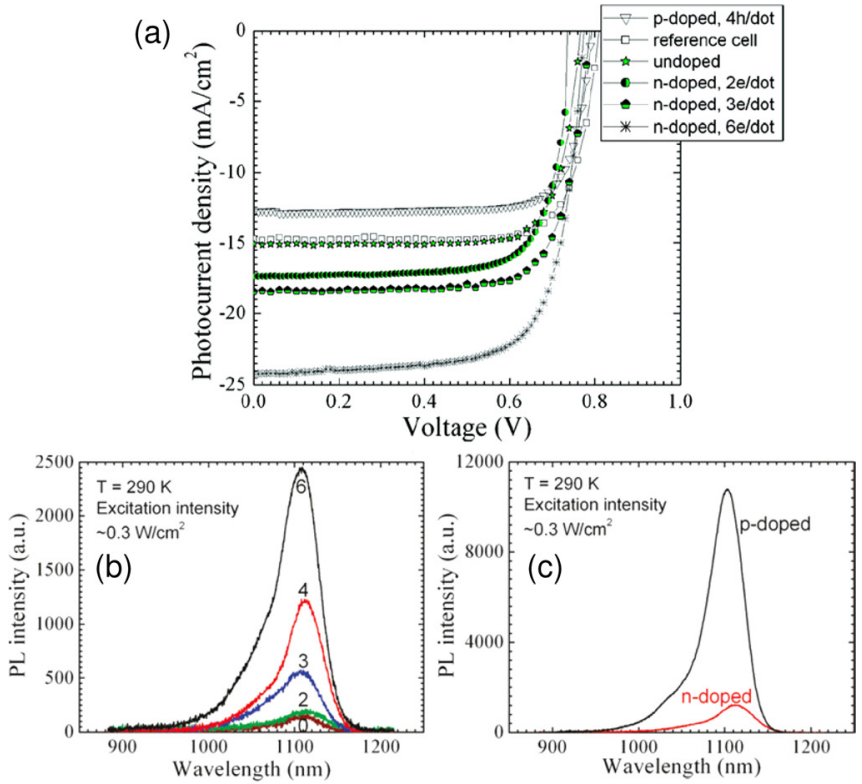

(d)

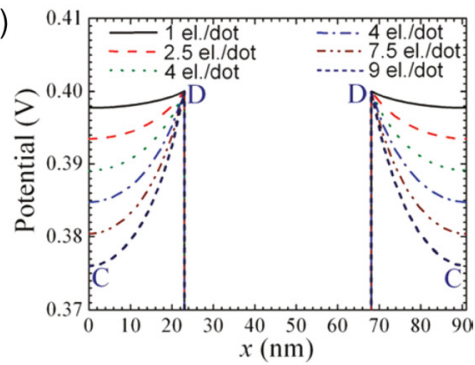

FIG. 41. (a) $J-V$ characteristics under 1 sun (AM $1.5 \mathrm{G}$ ) at $100 \mathrm{~mW} / \mathrm{cm}^{2}$ of QDSCs as a function of doping: $p$-doped QDSC with 4 holes per QD, GaAs reference cell, undoped QDSC, $n$-doped QDSCs with 2, 3, and 6 electrons per QD. (b) Dependence of the PL at RT on the doping level for undoped device and $n$-doped devices with 2,3, 4, and 6 electrons per QD and (c) comparison of PL in $p$ - and $n$-doped devices with the same level of doping (4 carriers per QD). (d) Calculated potential barriers for electrons in QD plane as a function of built-in electron charge using multiband $k \cdot p$. Reprinted with permission from Sablon et al., Nano Lett. 11, 2311 (2011). Copyright 2011 American Chemical Society.

two electrons to QD excited states; then due to strong interelectron interaction in a $\mathrm{QD}$, one of these electrons transfers to the conducting state and the other transfers to a low-energy state. Figure 41(b) shows the dependence of the PL on the doping level in $n$-doped samples. The PL intensity substantially increases with doping. This observation is associated with enhancement of intradot relaxation processes via electron-electron and hole-electron scattering in QDs due to electrons trapped in the QDs, which facilitate the carrier recombination. The PL intensity enhancement might be also a consequence of thermal decoupling between the barrier and QD region due to a potential barrier formed between QD and the barrier region, as shown in Fig. 41(d). PL of $p$ - and $n$ doped samples with the same level of doping, which corresponds to four carriers per QD is shown in Fig. 41(c). As seen, the PL intensity of $p$-doped sample is approximately 8 times of that of $n$-doped sample. Thus, the $p$-doping substantially enhances capture and relaxation processes and increases the recombination losses. This can be again explained by the potential barrier formed between QD and the barrier. As shown in Fig. 41(d), for an $n$-doped sample, the potential barriers formed around QD planes suppresses the fast electron capture processes and accelerates the capture of holes. For a p-doped QD sample, the opposite effect would be expected that facilitates fast electron capture while suppressing hole recombination.

Yang et al. have fabricated a direct Si-doped InAs/GaAs QDSC where the effect of Si-doping on the properties of the InAs QDSC was investigated. ${ }^{174} \mathrm{~A}$ series of samples with a single layer of InAs QDs under different doping levels $\left(4 \times 10^{10} \mathrm{~cm}^{-2}, 1 \times 10^{11} \mathrm{~cm}^{-2}, 5 \times 10^{11} \mathrm{~cm}^{-2}, 1 \times 10^{12} \mathrm{~cm}^{-2}\right)$ were prepared. Figure 42(a) shows the RT-PL spectra of the InAs QDs with different Si-doping levels. The QDs with a Sidoping level of $5 \times 10^{11} \mathrm{~cm}^{-2}$ exhibit the strongest PL peak intensity, which is about 30 times stronger than that of the undoped QD sample. The enhanced PL signal was attributed to two possible reasons. One is due to the shortened $\mathrm{Si}$-As bond distance which relaxes the strain of the InAs QD and the other reason is that the defect may have been inactivated by the $\mathrm{Si}$ doping. $^{31,173}$ The $J-V$ characteristics in Fig. 42(b) of the QDSCs show that QDSCs with an optimized Si-doping level of $5 \times 10^{11} \mathrm{~cm}^{-2}$ show a significant increase of the $V_{\mathrm{OC}}$ up to $0.84 \mathrm{~V}$. The efficiency of QDSC with the optimum Si-doping level is dramatically improved from $11.3 \%$ to $17.0 \%$.

Lam et al. have recently reported on similar voltage recovery in charged InAs/GaAs QDSCs. ${ }^{175}$ During QD growth, direct Si-doping was carried out with dopant
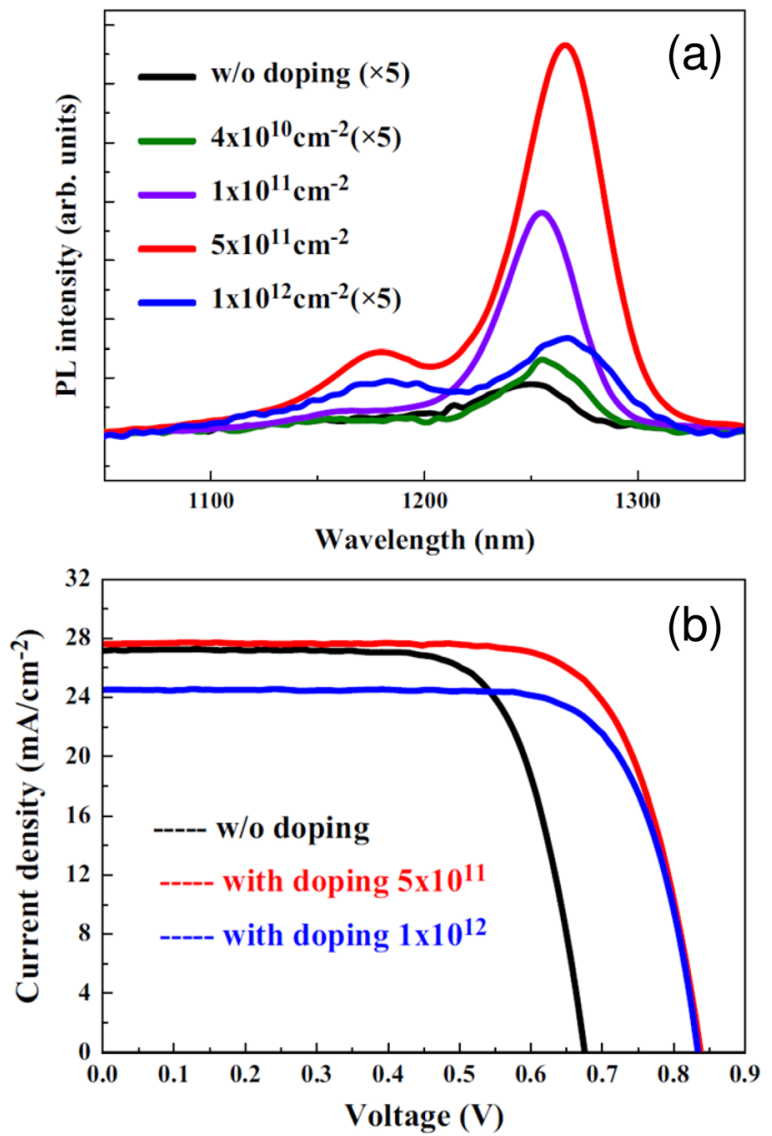

FIG. 42. (a) RT-PL spectra of InAs QDs with different Si-doping levels. (b) $J-V$ characteristics of each QDSC under 1 sun illumination. Reprinted with permission from Yang et al., Sol. Energy Mater. Sol. Cells 113, 144 (2013). Copyright 2013 Elsevier B.V. 
densities of $0,6,12,18$, and 24 electrons (e) per QD. From the $J-V$ characteristics analysis shown in Fig. 43(a), it was found that with increasing doping, the $J_{\mathrm{SC}}$ gradually decreases from $17.2 \mathrm{~mA} / \mathrm{cm}^{2}$ for the $0 e$ QDSC to $14.3 \mathrm{~mA} /$ $\mathrm{cm}^{2}$ for the 24e QDSC. On the other hand, with increasing doping concentration in the QDs, the $V_{\mathrm{OC}}$ was gradually improved. The $V_{\mathrm{OC}}$ increases from $0.777 \mathrm{~V}$ for the $0 e$ QDSC to $0.882 \mathrm{~V}$ after Si-doping with $18 e$ QDSC. The PL intensity increases with higher doping concentration in the QDs at RT (shown in Fig. 43(b)). The enhanced emission of doped QDSCs suggests that PL quenching is suppressed by increasing the thermal activation energy after Si-doping. They have proposed the following model to explain the charge effect between the wetting layer and the InAs QD. The Si dopants in the wetting layer supply free carriers to the QDs to make the QDs charged negatively and the wetting layer charged positively. As a result, a potential barrier formed at the interface between QD and wetting layer increases with doping density and subsequently decouples the electrons thermalescaping from the QDs.

\section{Concentrated photovoltaic (CPV) characterization}

Conversion efficiency of the QD-IBSC strongly depends on the bandgap of the host material, band profile alignment, the position of the IB, density of states in the IB, as well as carrier filling in the IB, as shown in Secs. III B 1-III B 3. In
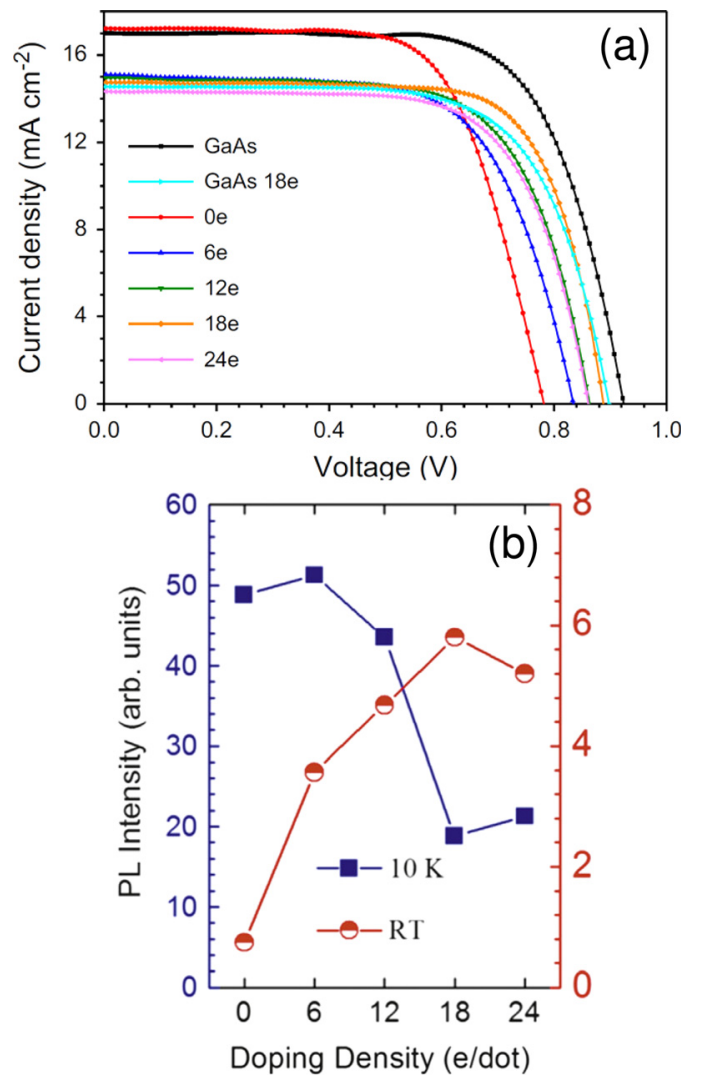

FIG. 43. (a) $J-V$ measurements obtained under 1 sun (AM 1.5G) illumination for Si-doped QDSCs, GaAs cell, and $18 e$ GaAs cell. (b) Integrated PL intensity for Si-doped QDSCs with $0 e, 6 e, 12 e, 18 e$, and $24 e$ per QD measured at $10 \mathrm{~K}$ and RT. Reprinted with permission from Lam et al., Nano Energy 6, 159 (2014). Copyright 2014 Elsevier B.V. this section, the efficiency of IBSC under solar concentration is considered. First, some simulation results showing the efficiency recovery of the InAs QD-IBSC under light concentration are presented, followed by some experimental results related to the characterization of InAs QD-IBSC under concentration.

InAs QD-IBSCs which commonly suffer from small absorption and low density of QDs leading to lowering of $V_{\mathrm{OC}}$ and efficiency are expected to recover faster and perform better under concentrated illumination operation. ${ }^{176}$ InAs QD-IBSCs require light concentration to ensure the carrier photo-generation outperforming the recombination processes via the IB. The areal density of QDs has direct influence on photo-generation and recombination via IB because the density of states of the IB $\left(N_{\mathrm{ib}}\right)$ is linked to areal density as: $N_{\mathrm{ib}}=N_{\mathrm{s}} N_{\mathrm{st}} / W$, where $N_{\mathrm{s}}$ is the areal density of the QDs, $W$ is the intrinsic layer width, and $N_{\text {st }}$ is the stacking number of the QD layers. ${ }^{177}$ Assuming $E_{\mathrm{g}}$ of $1.40 \mathrm{eV}$ for GaAs host material while varying the location of IB energy level, based on the detailed balance theory the recombination and generation rates for InAs/GaAs QDSC under different positions of IB were calculated and the results were shown in Fig. 44(a). The quantum dot areal density in Fig. 44 corresponds to a total QD density defined as $N_{\mathrm{s}} \times N_{\mathrm{st}}$. The absorption cross sections were set as $3 \times 10^{-14} \mathrm{~cm}^{2}$ for both optical transitions between VB to IB and IB to $\mathrm{CB}$, which is the same as in the previous detailed balance calculations. ${ }^{23,177} \mathrm{It}$ is clearly seen here that if the areal density of QD is below $1 \times 10^{16} \mathrm{~cm}^{-2}$, the net generation rate which was defined as $G_{n e t}=G_{\mathrm{IC}}-R_{\mathrm{IC}}$ (see Eq. (14)) becomes negative with deepening the location of IB in the QD potential region. Since the today's experimentally reachable highest density of InAs QD is still at the order of $10^{12} \mathrm{~cm}^{-2}$, the recombination process will inevitably dominate the carrier dynamic behavior while resulting in a "negative loss zone" (see Fig. 44(a)). However, the situation is greatly improved when the cell is under light concentration. Figure 44(b) shows the net generation rate variation with the light concentration ratio for the case where the IB is located at $0.2 \mathrm{eV}$ below the $\mathrm{CB}$ edge. It shows that the net generation rates turn positive after 10 suns and the "negative loss zone" was disappeared. When the concentration exceeds 1000 suns, positive net generation rate can be expected even for the QD with areal density of $1 \times 10^{12} \mathrm{~cm}^{-2}$. Figure $44(\mathrm{c})$ shows the efficiency recovery under concentration and similar tendency to the net generation rate was found, indicating the important role of light concentration to guarantee the optimal operation of InAs QD-IBSC. Simulation results here are consistent with the results reported in literature. ${ }^{177,178}$

Experimental study of InAs/GaAs QD-IBSC under light concentration has gradually drawn attention. ${ }^{180}$ Takata et al. have reported that in a well-developed cell, ${ }^{181}$ a 50-layers stacked InAs/GaNAs QDSC with $\sim 15.7 \%$ efficiency at 1 sun has been shown to improve to efficiency of $\sim 20.3 \%$ at 100 suns and $\sim 21.2 \%$ at 1000 suns illumination. ${ }^{141}$ Lantratov et al. have reported CPV study results by using four types of different InGaAs QDSCs: with the QD media in the middle of the $i$-GaAs region ( $i$-QDSC); with the same structure but using the Bragg reflector (BR-QDSC); with the QD media 
(a)

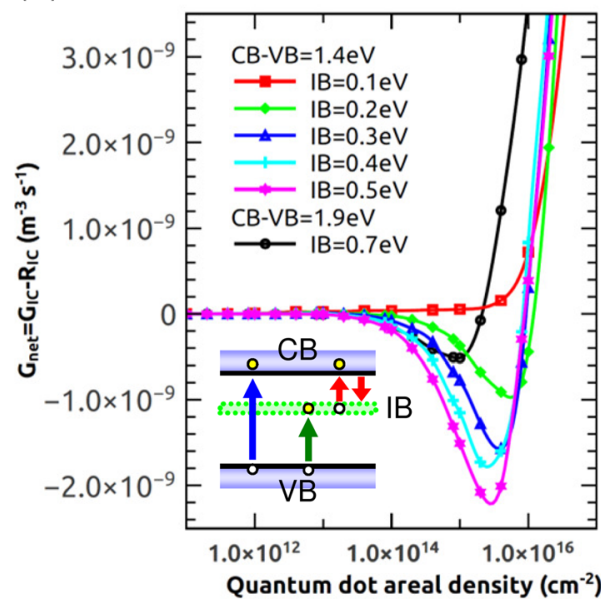

(b)

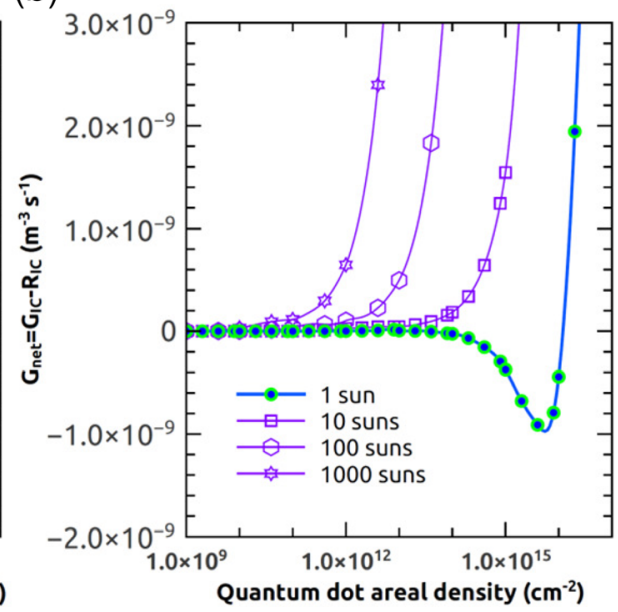

(c)

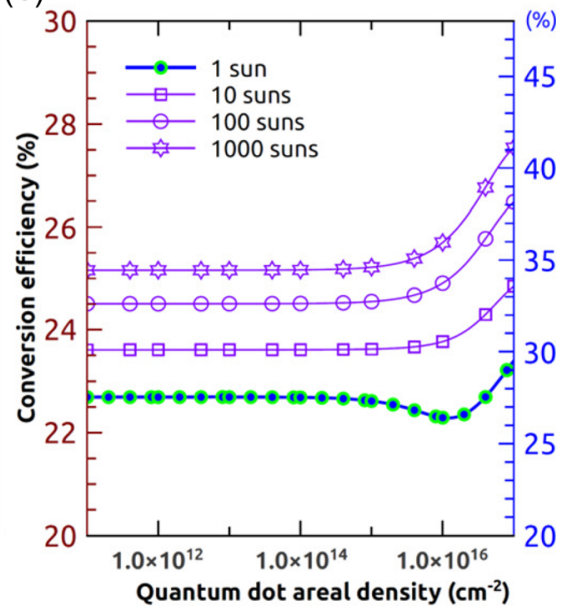

FIG. 44. Detailed balance simulation results of the dependence on the areal density of InAs QD. (a) $G_{n e t}=G_{\text {IC }}-R_{\mathrm{IC}}$ variation for the IB energy positions relative to the bottom of the CB. Inset: Detailed balance simulation model. (b) $G_{\text {net }}$ and (c) conversion efficiency under 1 sun (left hand scale in (c)) and $10-1000$ suns (right hand scale in (c)) for the case the IB is located at $0.2 \mathrm{eV}$ below the $\mathrm{CB}$ edge.

between $i$-GaAs region and $n$-GaAs base (base-QDSC); and in a conventional design (without QDs) keeping the $i$-region width of $130 \mathrm{~nm}$ (conv.-SC). $V_{\mathrm{OC}}$ dependence on the concentration ratio was plotted in Fig. 45. They have demonstrated the efficiency for BR-QDSCs reached at the level of 25\% (30 suns AM 1.5D). ${ }^{121}$

Figure 46(a) shows the test results of the InAs QDs at higher concentration reported by Hubbard et al. ${ }^{179}$ It was found that the QDSCs showed enhanced $J_{\mathrm{SC}}$ than the GaAs reference cell. A high level injection induced superlinear trend was also observed in all cells. As expected, the QDSC containing 20-layers of InAs QD gave the highest $J_{\mathrm{SC}}$, $7.53 \mathrm{~A} / \mathrm{cm}^{2}$ at concentration ratio of 440 suns, which is $11 \%$ increase compared to the current of the reference cell at the same concentration. By fitting the results of $V_{\mathrm{OC}}$ vs. concentration ratio, it was found that the ideality factor was 1.4 , which indicates the cells were affected by the SRH recombination in the quasi-neutral region. By plotting the $J_{\mathrm{SC}}-V_{\mathrm{OC}}$ curves shown in Fig. 46(b) which, in principle, reflects partially the dark diode property of the cells, the results showed that the QDSC saturation current was approximately two orders magnitude higher than the reference cell. This can be either attributed to a nonradiative recombination in

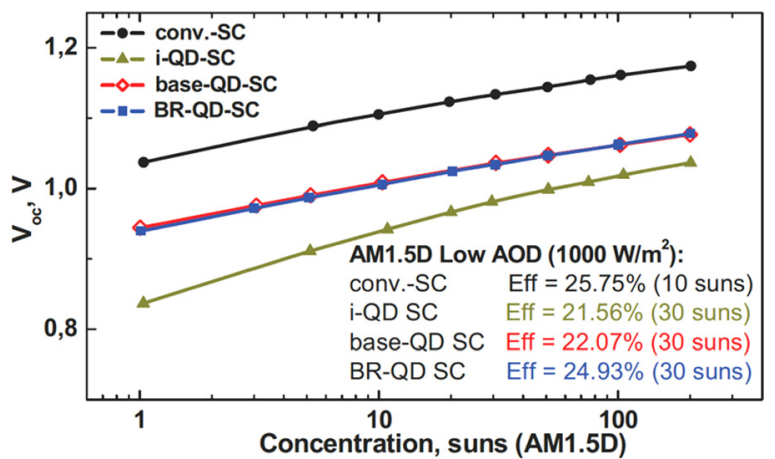

FIG. 45. The dependencies of $V_{\mathrm{OC}}$ on concentration of incident solar spectrum for conventional solar cell, $i$-QDSC, base-QDSC, and BR-QDSC. Reprinted with permission from Lantratov et al., Adv. Sci. Technol. 74, 231 (2010). Copyright 2010 Trans Tech Publications, Inc.
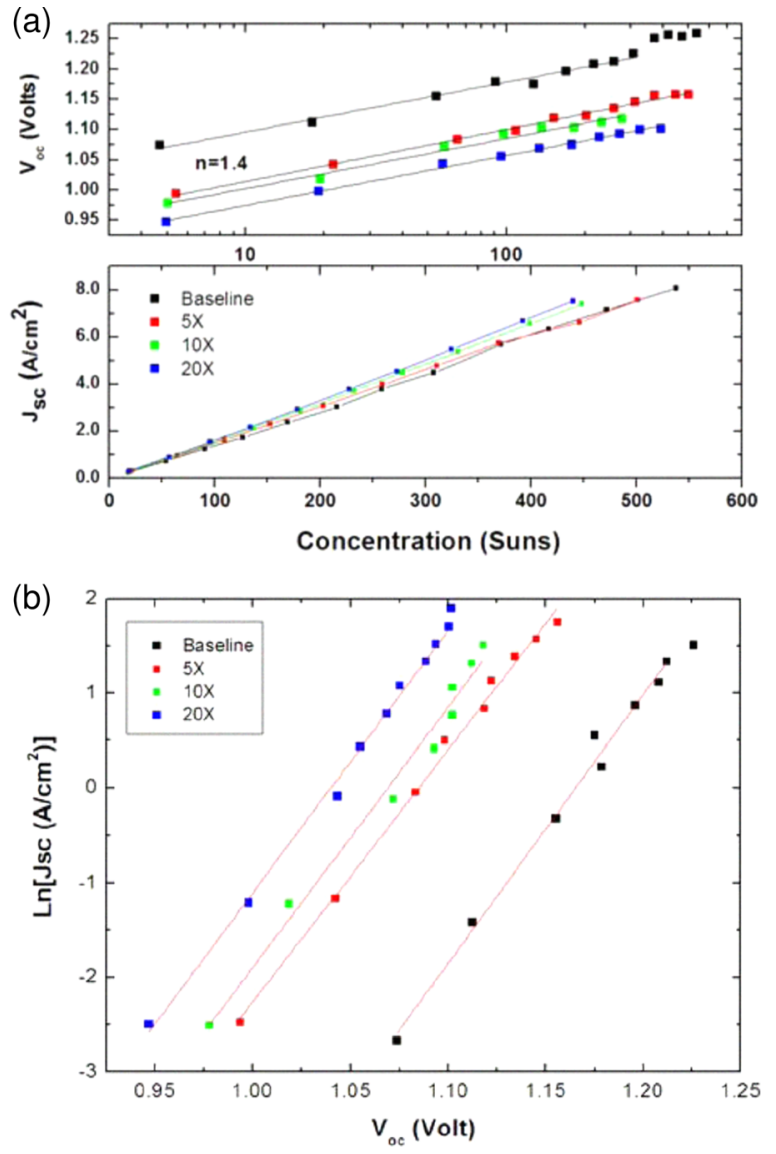

FIG. 46. (a) $\mathrm{AM} 1.5 \mathrm{D} V_{\mathrm{OC}}$ and $J_{\mathrm{SC}}$ vs. concentration for the reference cell and QDSC. (b) Concentrated light $J_{\mathrm{L}}-V_{\mathrm{OC}}$ characteristics of both the QDIBSC and the GaAs reference cell. The reference cell without InAs QDs had a $100 \mathrm{~nm} \mathrm{GaAs}$ intrinsic region, while cells with QDs had an array of 5, 10, and 20-layers of undoped InAs QDs grown in the $i$-region using optimized QD growth conditions. Reprinted with permission from Hubbard et al., "Characterization of quantum dot enhanced solar cells for concentrator photovoltaics," in Proceedings of the 34th IEEE Photovoltaic Specialists Conference (2009), pp. 000090-000095. Copyright 2009 IEEE. 
the quasi-neutral region or the results of the lowered bandgap by the increased number of QD layers.

Linares et al. have reported temperature variable measurement of light-induced current density $\left(J_{\mathrm{L}}\right)-V_{\mathrm{OC}}$ pairs at different concentration ratios for InAs/GaAs QD-IBSC. ${ }^{182}$ Figure 47(a) shows the RT experiment results, where the voltage recovery effect is not observed due to large radiative and nonradiative recombination in the QD region. Figure 47 (b) shows the results from the measurement at $150 \mathrm{~K}$, in which the IBSC voltage recovery is appearing due to the reduced nonradiative recombination by decreasing the temperature. The $V_{\mathrm{OC}}$ of the InAs QDSC finally reached the same $V_{\mathrm{OC}}$ as the GaAs reference cell at $77 \mathrm{~K}$, thus verifying the proposed IBSC voltage preservation principle. ${ }^{176}$

A detailed CPV study of InAs QD-IBSC was recently performed by Sogabe et al. with special focus on revealing the IB dynamics under light concentration. ${ }^{183}$ They have reported the first demonstration and testing of InAs/GaAs QD-IB CPV module. Figure 48(a) shows a picture of the fabricated CPV module, which contains two separate parts with four series-connected GaAs control sub-modules on one side and four InAs/GaAs QD-IB CPV sub-modules on the other side. The InAs/GaAs QD-IB CPV and GaAs control cells were fabricated by adopting a $p-i-n$ cell structure on
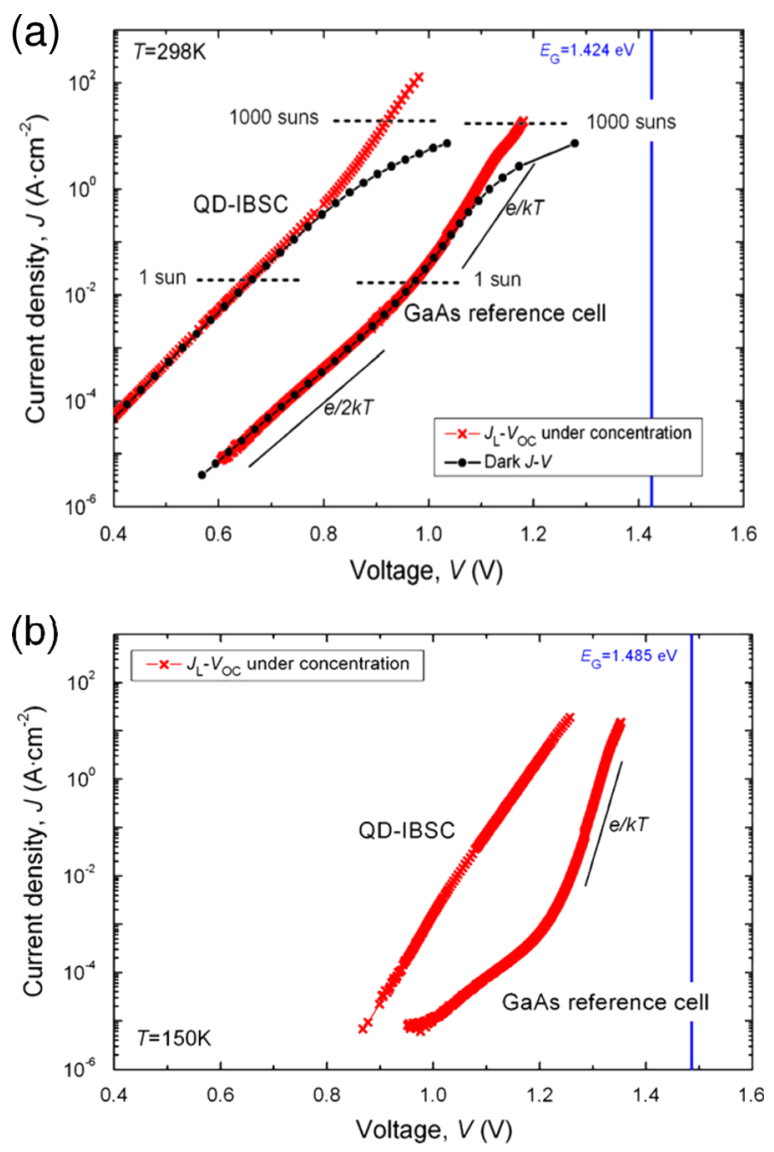

FIG. 47. (a) Concentrated light $J_{\mathrm{L}}-V_{\mathrm{OC}}$ characteristics of both the QD-IBSC and the reference cell at different temperatures. Dark $J-V$ and concentrated light $J_{\mathrm{L}}-V_{\mathrm{OC}}$ characteristics measured at $298 \mathrm{~K}$. The concentration levels are indicated with horizontal gray dashed lines. (b) The concentrated light $J_{\mathrm{L}}-V_{\mathrm{OC}}$ characteristics measured at $150 \mathrm{~K}$. Reprinted with permission from Linares et al., Sol. Energy Mater. Sol. Cells 98, 240 (2012). Copyright 2012 Elsevier B.V. (a)
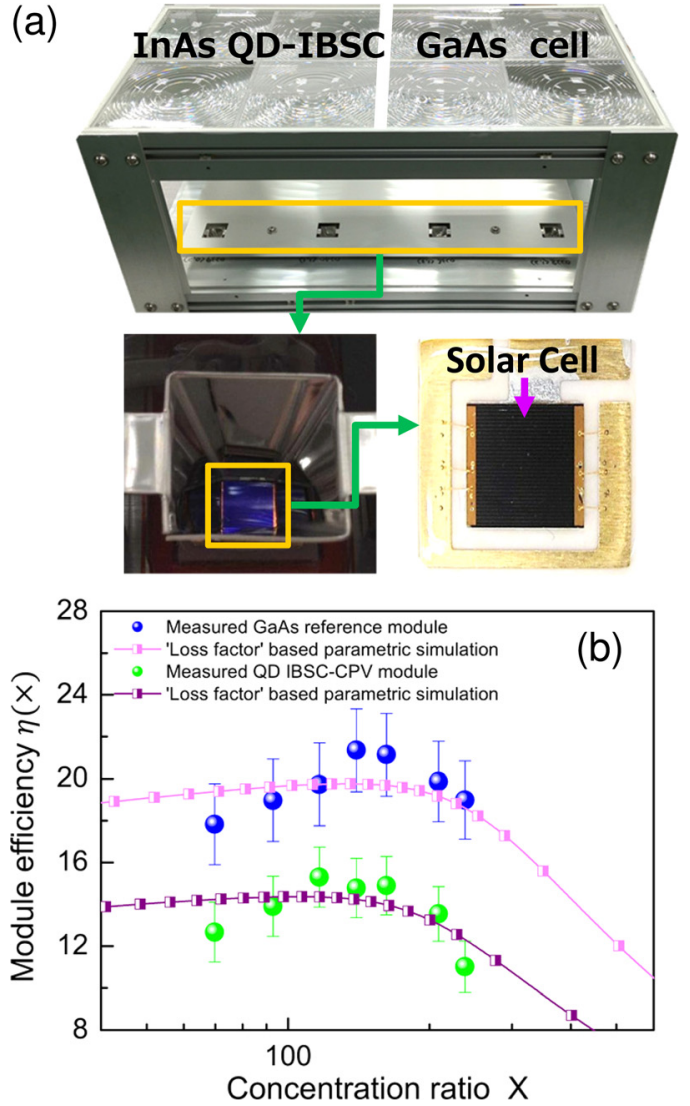

FIG. 48. (a) Picture of the fabricated CPV module and the submount of the IBSC on aluminum heat sink plate; (b) conversion efficiency under different concentration ratio, $X$. Fitted results based on "loss factor" parametric method, are also presented. Reprinted with permission from Sogabe et al., Sci. Rep. 4, 4792 (2014). Copyright 2014 Nature Publishing Group.

n-type GaAs (001) substrate using MBE. The QD-IB CPV module characterization has been performed at RT using HELIOS 3198, a standard CPV module measurement system with the light source from a flash lamp. The conversion efficiency of $19.2 \%$ has been obtained for GaAs reference module and $15.3 \%$ for a 10 -layers stacked InAs/GaAs QD-IB CPV module at 116 suns under IEC62108 standard as shown in Fig. 48(b).

In order to identify the dynamics of IB under light concentration, a well calibrated investigation was conducted to reveal the dependence of $J_{\mathrm{SC}}$ and $V_{\mathrm{OC}}$ of the InAs/GaAs QDIB CPV module on the concentration ratio, $X$. The data shown in Fig. 49(a) are the normalized data for $J_{\mathrm{SC}}(X) / J_{\mathrm{SC}}$ $(X=1$ sun). It was found that the increase rate defined as $d J_{\mathrm{SC}}(X) / d X$, of the GaAs control module is 0.0061 and is 0.0070 for the QD-IB CPV module. From the theoretical analysis, an accelerated increase of $d J_{\mathrm{SC}}(X) / d X$ in the QDIB CPV module is one of the decisive signatures of the proper electron dynamics in an IBSC under light concentration. $V_{\mathrm{OC}}$ of the QD-IB CPV module shown in Fig. 49(b) is found about $0.25 \mathrm{~V}$ lower than in the GaAs control module. The amount of this reduction is mainly due to the fact that recombination via the IB is still prominent. In addition, the contribution to the voltage reduction may also come partially from the greater thermalization, i.e., larger dark saturation 
(a)

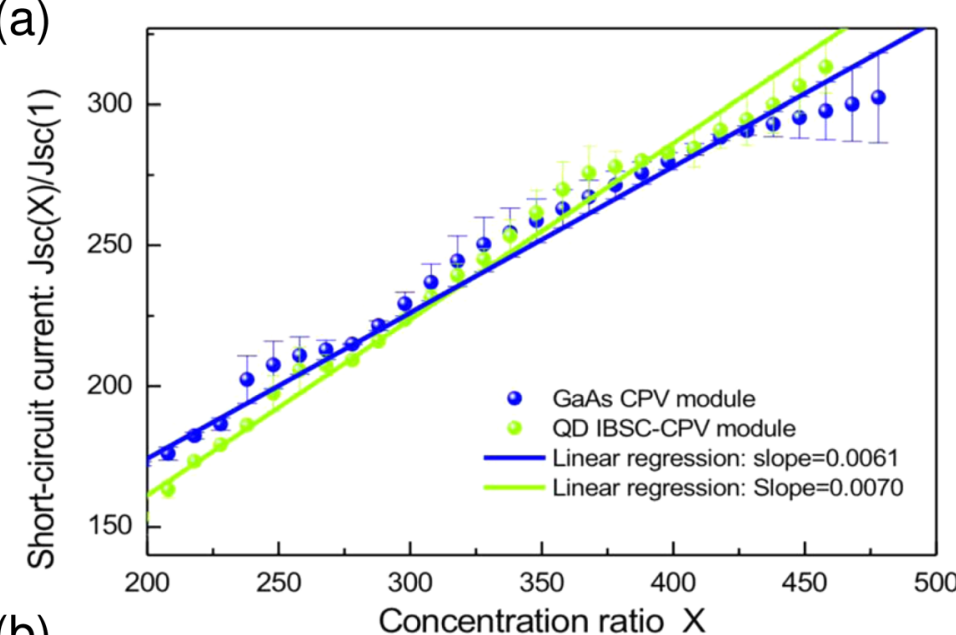

(b)

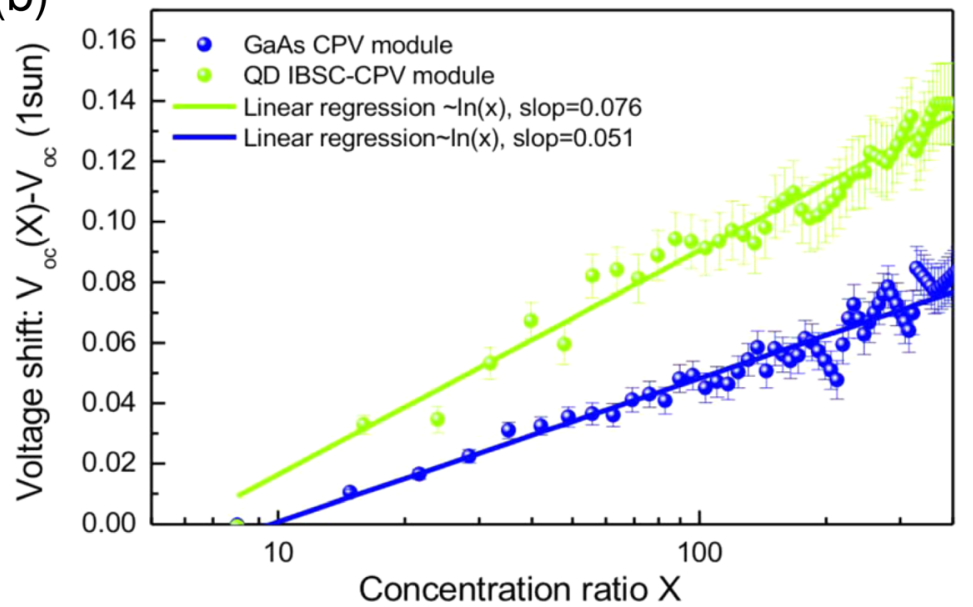

(c)

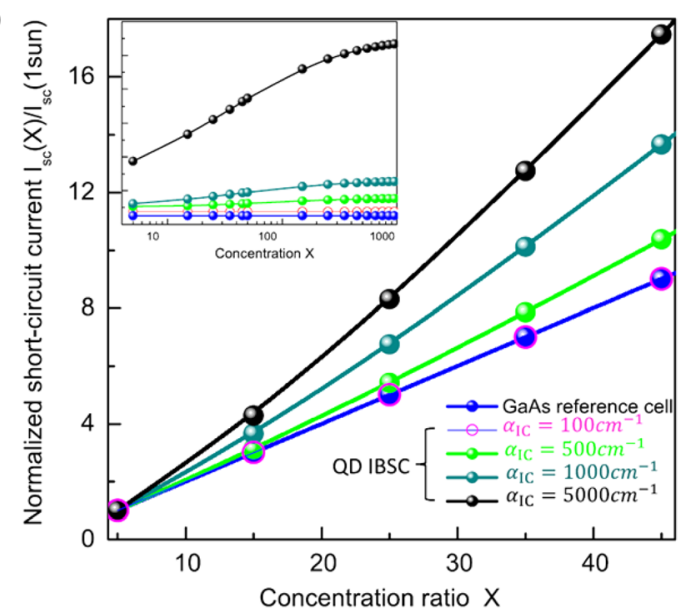

(d)

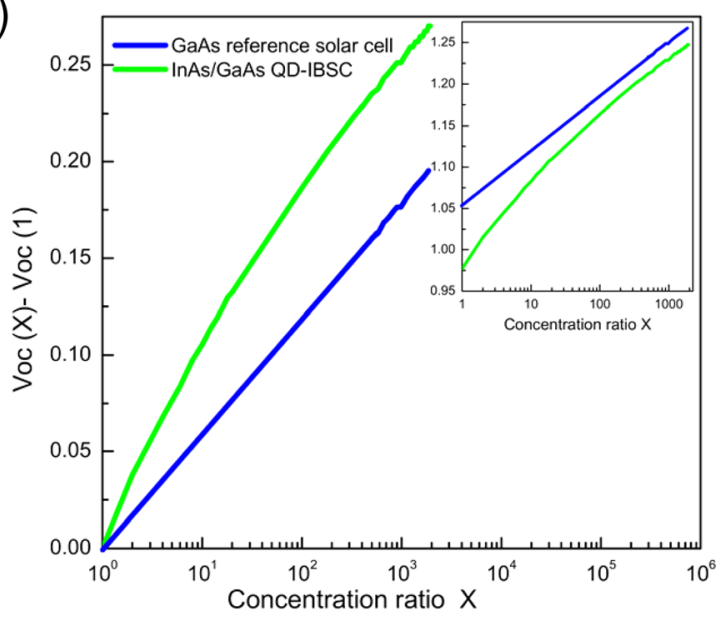

FIG. 49. (a) Linear regression fitting of $J_{\mathrm{SC}}(X)$ with least square error bars. (b) Linear regression fitting of $V_{\mathrm{OC}}(X)$ with least square error bars. (c) Calculated $J_{\mathrm{SC}}(X) / X$ for QD-IBSC under different absorption coefficients of the transition from IB to CB. (d) Calculated $V_{\mathrm{OC}}$ for QD-IBSC and GaAs control cell under different concentration ratio X. Adapted with permission from Sogabe et al., Sci. Rep. 4, 4792 (2014). Copyright 2014 Nature Publishing Group.

current due to the reduced VB-CB energy gap in an InAs QD-IBSC.

A combined multiband $k \cdot p$ and drift-diffusion simulation results are plotted in Figs. 49(c) and 49(d) showing the dependence of $V_{\mathrm{OC}}$ and $J_{\mathrm{SC}}$ of QD-IB CPV module on concentration ratio $X$. The increased rate of $V_{\mathrm{OC}}$ and $J_{\mathrm{SC}}$ are much larger for the QD-IB CPV than for the GaAs control cell. Further simulation found that when the IB to $\mathrm{CB}$ absorption coefficient is above $500 \mathrm{~cm}^{-1}$, which is below the experimentally reported absorption coefficient for the IB to $\mathrm{CB}^{184}$ reviewed in the Sec. III D 3 or for the VB to IB, ${ }^{185}$ the difference in increase rate of $d J_{\mathrm{SC}}(X) / d X$ becomes clearly distinctive, indicating the validity to study the IB dynamics under $\mathrm{CPV}$ condition.

In short, fabrication technologies and characteristics of III-V based QDSC are reviewed in this section. The lattice strain induced by S-K growth QDs deteriorates the crystalline quality of QDSC. Compensating for the strain in QD layers with SCLs that exerts an opposite biaxial strain avoids the generation of dislocations by accumulated strain in multi-stacked QD structure, thereby improving the cell performances. A type-II energy band alignment using $\mathrm{Sb}$ enables QD system with an extremely long carrier lifetime. Further, the irradiating $\mathrm{Sb}$ to the buffer layer has the effect of increasing QD density. Doping, including both direct and modulation doping into the InAs QDs, showed positive effects on the electrical and optical properties of the QDIBSC when the doping concentration is controlled within an optimal range. PL signal shows monotonically increase with increase of $n$-type doping concentration in the QDs. From the $J-V$ characterization results, most of the doped InAs QDIBSC showed significant recovery of $V_{\mathrm{OC}}$ due to either the photon induced inactivation of the defects or the built-in field which suppresses the electron recombination. The enhancement of $J_{\mathrm{SC}}$ at RT with doping is also reported and is attributed to the effects such as TSPA. InAs QD-IBSCs show better performance under moderate light concentration condition. Lack of QD areal density can be effectively compensated by the light concentration. A "negative loss zone" due to the negative net carrier generation disappears after the light concentration. Combined with low temperature effect which can effectively suppress the carrier recombination, a noticeably fast recovery of $V_{\mathrm{OC}}$ was found for InAs QDIBSC at $77 \mathrm{~K}$ and finally matched the $V_{\mathrm{OC}}$ of GaAs cell under concentration ratio of 450 suns. Meanwhile, it also demonstrates that the IB dynamics can be examined under light concentration via an approach to detect the dependence of increase rate of $J_{\mathrm{SC}}$ and $V_{\mathrm{OC}}$ on the concentration ratio for 
QD-IB CPV module. Confirmation of the IB dynamics in IBSC-CPV module has significant impact on the future field application for the QD-IBSC.

\section{IB materials with transition metals}

Because the IB research direction has been first based on a pure concept, a fraction of the research has been directed at finding suitable materials to embody the concept, based on first principles. With the exception of a few papers on $\mathrm{TiO}_{2}$, the entire search for new compounds has been focused on modifications of known semiconductors developed for optoelectronic applications.

As it turns out, all the technologically relevant compounds are tetrahedrically coordinated, derived from the diamond crystal structure and are characterized by high carrier mobilities, possibility to control doping level, and achieve long carrier lifetimes. In these compounds, the VB has dominantly an anionic character deriving from $p$-orbitals, while the CB derives predominantly from $s$-states of the cations. For symmetry reasons, and because of their reduced spatial extension, impurities with $d$-states open shells (transition metals) favor the creation of narrow bands with non-bonding character, and therefore whose levels will sit in the middle of the gap. This approach fundamentally differs from that of HMAs, another intensively investigated route to IBSC. As it turns out, transition metals can also impart new properties to semiconductors, which might be relevant for IBSC, and we first turn to examine those.

\section{Dilute magnetic semiconductors}

As discussed earlier, long excited state lifetimes in the IB are important for photovoltaic conversion applications, as they improve the chance that the photo-generated carriers will be promoted to CB. An example of long lifetimes is provided by organic molecules that display long lived triplet states. ${ }^{15}$ In inorganic semiconductors, there is not a straightforward equivalent long lifetime spin state since one always finds free carriers of both spin states for which recombination is readily allowed. However, a recent class of material has emerged recently that may display the desirable properties: dilute magnetic semiconductors (DMSs). They are promising compounds for spintronics, especially since some have been found to become ferromagnetic at room temperature. ${ }^{186}$

The use of ferromagnetic DMS to mimic triplet states in bulk inorganic semiconductors for photovoltaic power conversion has therefore been proposed recently. ${ }^{16,187}$ In such compounds, the spin degeneracy of the bands is lifted, opening the way for configurations favorable for the operation of IB devices (see Fig. 50) where recombination through the IB is suppressed by spin selection rules or by low occupancy of states involved in the allowed recombination. Previously, proposals have been made to better utilize the low energy part of the incident solar spectrum by using intermediate level semiconductors (ILSCs) either with localised states ${ }^{4}$ or delocalised ones. ${ }^{2,3}$ The proposed DMS-based scheme with three absorption bands, as shown in Fig. 50, can provide a

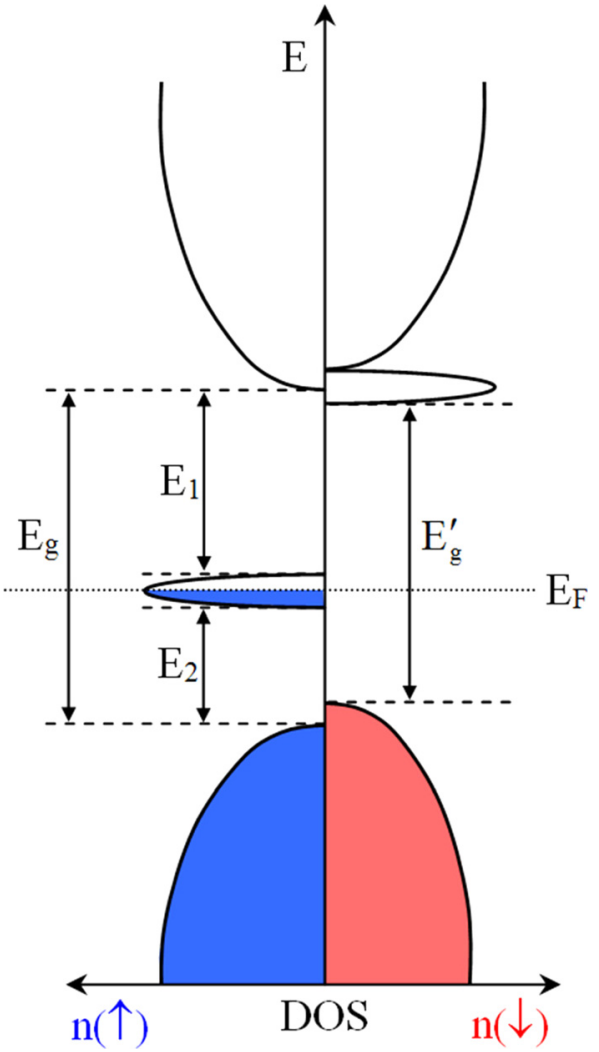

FIG. 50. Schematic density of states of a DMS (spin up and down) optimal for photovoltaic conversion. In most systems, the Fermi level is located in the middle of the $3 d$ induced states (generally $t_{2 g}$ ). This level defines the optical threshold for a transition from the VB or to the CB. In the class of compounds considered, the VB has a $p$ character and the $\mathrm{CB}$, an $s$ character. This imparts to transitions from the VB to the $t_{2 g}$ state a stronger oscillator strength than to those from $t_{2 g}$ to the $\mathrm{CB}$, and therefore provides a way to achieve an optimal allocation of the photons among the various transitions when the $t_{2 g}$ is located at $2 / 5$ of $E_{g}$ from the CB. Reprinted with permission from Olsson et al., Phys. Rev. Lett. 102, 227204 (2009). Copyright 2009 American Physical Society.

pathway for the realization of solids with the desired optical properties.

The potential efficiency of solar cells based on DMS has been derived from the photon and electron balance equations, as usual. In the present case, these were computed within the Boltzmann transport formalism, where additional selection rules based on electron spin have to be considered in the case of DMS. ${ }^{16}$ Detailed assumptions related to the calculations can be found in Ref. 16 and its supplementary materials.

All efficiencies reported were related to standard AM 1.5 solar illumination. ${ }^{188}$ In both IL type devices (DMS and ILSC), the sensitivity to precise energy level positions was not found to be very large as the optimum is wide. This is illustrated by the contour plots of Fig. 51 where isoefficiency lines are plotted as a function of both the smallest and largest transition energies: efficiencies up to $52 \%$ are achievable with DMS cells (Table I). Remarkably, non-ideal DMS compounds suffering from nonradiative recombination still offer excellent efficiency expectations. The efficiency decrease in an ILSC is very similar to a DMS but the possibility of attaining a high level of radiative recombination without a blocking mechanism is very low with the former 


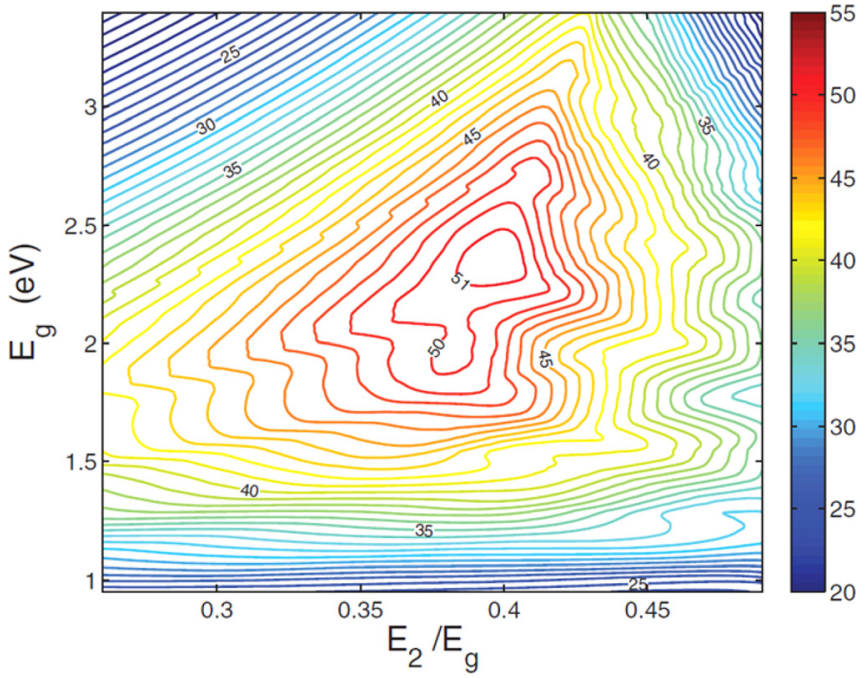

FIG. 51. Contour plots of the conversion efficiency under 1 sun illumination with AM 1.5 spectrum by a DMS solar cell as a function of $E_{2}$ and $E_{g}$. Contours are spaced by $1 \%$. The lowest corresponds to $20 \%$ conversion efficiency, while the central one corresponds to $52 \%$ efficiencies. For comparison, under the same assumptions, a semiconductor in a single-junction photovoltaic device would be limited to $31 \%$ (for which the optimal bandgap is $1.31 \mathrm{eV}$ (Ref. 5)). Adapted with permission from Olsson et al., Phys. Rev. Lett. 102, 227204 (2009). Copyright 2009 American Physical Society.

as illustrated in the literature. ${ }^{105}$ These computations gave the optimal transition energies. Considering Figs. 50 and 51 as well as the ordinary physical guidelines with respect to photovoltaic device construction, the following design criteria for suitable DMS compounds were proposed: (i) $E_{g}$ in the 1.5-3.5 eV range, (ii) lift of $\mathrm{CB}$ minimum and $\mathrm{VB}$ maximum spin degeneracy by $0.1-0.2 \mathrm{eV}$ for efficient spin polarisation, (iii) existence of a ferromagnetic state with, a Curie temperature $T_{\mathrm{C}}$ greater than $300 \mathrm{~K}$, (iv) existence of a spin polarised IB at a suitable energy (about $2 / 5$ of the gap), with a high absorption coefficient for all transitions, and (v) an $E_{g}^{\prime}$ gap smaller than the $E_{g}$ (i.e., $\Delta E_{g}>0$ in Fig. 50). As noted before, ${ }^{105}$ efficient absorption on both transitions with the IB as the initial or final state will also require partially occupied IB, which in some cases can be provided by the transition element itself. Finally, this is a point not too often discussed in the context, some attention was also paid to the impact of the width of the IB.

\section{Lifetime recovery}

Investigating the causes for an increased nonradiative recombination imparted by the ILs, it was proposed ${ }^{6}$ that

TABLE I. Efficiencies and optimal values of absorption thresholds of DMS and ILSC solar cells for a given branching ratio of radiative to nonradiative recombination $\left(R_{r} / R_{n r}\right)$.

\begin{tabular}{lccccc}
\hline \hline $\mathrm{R}_{\mathrm{r}} / \mathrm{R}_{\mathrm{nr}}(\%)$ & Type & Eff $(\%)$ & $\mathrm{E}_{\mathrm{g}}(\mathrm{eV})$ & $\mathrm{E}_{2}(\mathrm{eV})$ & $\Delta \mathrm{E}(\mathrm{eV})$ \\
\hline 100 & DMS & 52.0 & 2.345 & 0.933 & 0.295 \\
100 & ILSC & 49.5 & 2.433 & 0.933 & 0 \\
1 & DMS & 45.7 & 2.336 & 0.943 & 0.393 \\
1 & ILSC & 42.7 & 2.469 & 0.948 & 0 \\
\hline \hline
\end{tabular}

under certain conditions, increasing the concentration of dopants could lead to an increase, rather than to the expected decrease, of the minority carrier lifetime.

The underlying reason for that lies with the process by which deep (mid-gap) impurities trigger nonradiative recombination through carrier capture followed by the excess energy dissipation through multiphonon emission, fairly well known for isolated impurities. In that case, the relaxation rate (via the capture cross section) is expected to decrease fast as the size of the defect wavefunction increases. Since metallic systems have delocalised states, this result was taken to imply that mid-gap metallic states might become a relatively inefficient route for nonradiative recombination. Then, a high impurity concentration, strong enough to cause an insulator to metal transition, could lead to lifetime recovery.

Indeed, some experimental validation of this was found studying Si highly doped with $\mathrm{Ti}^{189}$ and postulated in the case of heavy doping with chalcogens. ${ }^{190}$ It should be noted nevertheless that in many cases the converse is true: large defect concentrations do not decrease nonradiative recombination. More importantly, the introduction of a high density of dopants does not restore radiative recombination as the dominant mechanism. A careful examination of the underlying model, extending the multiphonon model to the case of large impurity concentrations, ${ }^{191}$ indeed confirmed that delocalised states in this case actually increase the trapping rates.

The above considerations do not exclude that under some conditions a trivial increase of the lifetime with increasing impurities could occur (when, e.g., the nature of the impurity states are varied, as when impurity complexes or precipitates get formed), but these are not expected to lead to a strong decrease of the nonradiative rates, and especially at levels close or below the radiative rates.

\section{Ab-initio computation of IB and DMS band structure}

IB semiconductors have been studied in the framework of DFT in order to determine the total energy difference between ferromagnetic and anti-ferromagnetic configurations, when relevant, as well as to obtain an approximate determination of the position of the IB. As is well known, the bandgaps predicted by DFT are systematically too small. As such, the IB position of the $3 d$ states as predicted by DFT can be questionable. In ordinary semiconducting systems, one can correct the DFT bandgap by applying the GW method $^{192}$ or by using exact exchange ${ }^{193}$ or self-energy corrected potentials. ${ }^{194}$ However, for simple systems, such as $\mathrm{Si}$ and $\mathrm{Ge}$, the introduction of a simple scissor operator that expands the bandgap without further affecting the band structure can very well approximate the GW correction of the band structure. ${ }^{192}$ In the ILV case, scissor cuts can a priori be placed above and/or below the IB. In comparison with experimental results, ${ }^{195,196}$ it was clearly seen that the bandgap correction should be fully applied above the Fermi level and thus the IB, ${ }^{16}$ but this rule has not been systematically applied to all investigations carried out in the field.

Some groups have computed the electronic density of states of transition metal doped semiconductors, and 


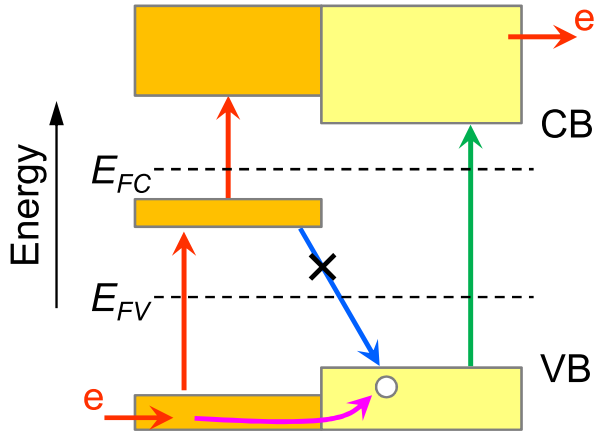

FIG. 52. Schematics of transitions in a DMS based IBSC.

especially GaAs and GaP doped by a variety of transition elements ( $\mathrm{Sc}, \mathrm{Ti}, \mathrm{V}$, and $\mathrm{Cr}$ ), ${ }^{197}$ albeit only at very high impurity concentration ( $25 \%$ of cation sites). In all cases, a well formed IB could be seen around mid-gap (finding subject to the limitations of the computation then used). II-VI binaries and I-III- $-\mathrm{VI}_{2}$ ternaries have been investigated as well ${ }^{198,199}$ with similar conclusions.

Interestingly, optical transitions oscillator strength and absorption coefficients related to the different transitions could have also been computed for many of these compounds, ${ }^{200-202}$ indicating that absorption coefficients in the range required for efficient IBSC operation (e.g., above $10^{4} \mathrm{~cm}^{-1}$ ) could be found in these material systems. For the requirement of reduced nonradiative recombination, we now turn to studies on DMS.

For DMS, the ferromagnetic- and anti-ferromagnetic calculations, respectively, two atoms in the supercell were substituted and given corresponding initial spins. Using standard Metropolis Monte-Carlo simulations on Ising spin system, ${ }^{187}$ determination of approximate Curie or Neel temperatures were also performed.
With the above rules in mind, the most II-VI, III-V binaries and some ternary tetrahedrally coordinated semiconductors were searched for candidates. For binaries, growth induced compressive strain can be used to lift the degeneracy from the top of the VB to enable an efficient spin polarisation, while selected ternaries such as I-III-VI 2 exhibit naturally this feature, ${ }^{203,204}$ and could also yield potentially interesting compounds, ${ }^{205,206}$ matching closely the ideal band structure (Fig. 52).

To find ferromagnetic candidates, doping with transition metals of the $3 d$ row was explored ab initio. Of more than a hundred compounds simulated (II-VI, III-V and I-III-VI with $E_{g}>1.5 \mathrm{eV}$ ), almost half were found to be ferromagnetically stable, and among those the search was narrowed in order to find the ideal type of electronic structure. Among all studied compounds only $2 \%$ were found to satisfy all criteria. This small number is itself a justification of the $a b$ initio screening of complex functional compounds having a long list of requirements to meet. A selection of the results of computation is shown in Fig. 53 and Ref. 243, showing the systematic shift to lower energies of the IB when transition metal atomic number increases.

Overall, in the midst of several promising compounds, the optimal one was found to be $\mathrm{Cr}$ doped zinc-blende AlP, an indirect bandgap semiconductor with an almost optimal bandgap of $2.43 \mathrm{eV}$. Substitution by $\mathrm{Cr}$ on the Al site was found to give rise to a near perfect electronic structure, from the point of view of the proposed design rules. ${ }^{16}$ The IB is present in only one spin channel and is there placed at 2/5 of the gap. In the other spin channel, the gap is somewhat closed by the spin split $d$ levels that end up at the CB minimum. An optimal conversion efficiency of $50 \%$ is calculated for AlP doped with $8 \% \mathrm{Cr}$ on the cationic sites, only a few percent is lower than the theoretical maximum conversion
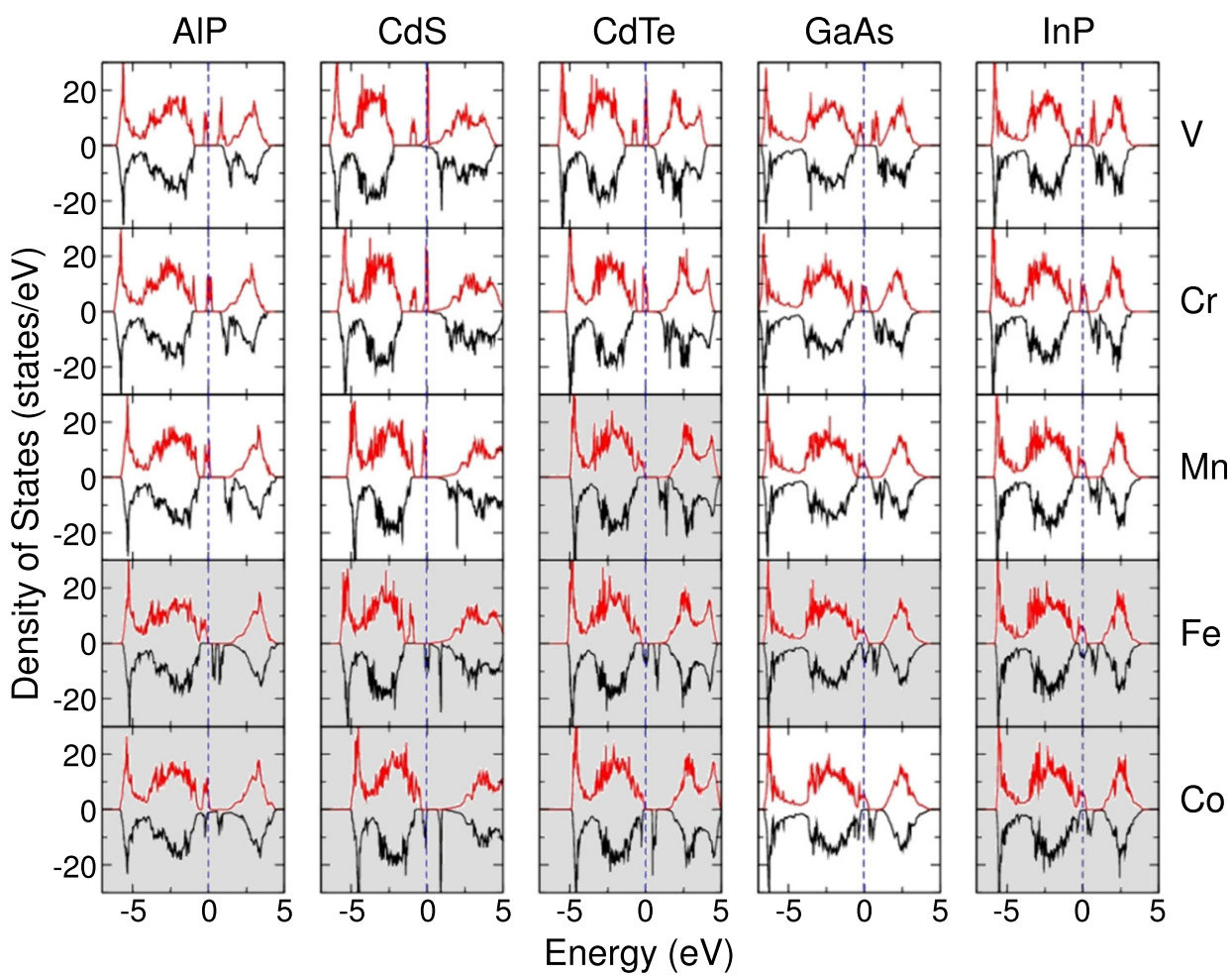

FIG. 53. Compilation of selected III-V and II-VI spin dependent density of states of compounds for main transition metal doping. Gray shaded compositions are anti-ferromagnetic, and therefore not relevant for DMS IBSC. (P. Olsson, C. Domain, and J.-F. Guillemoles, paper present at the 19th Quantsol Conference, 2007). ${ }^{243}$ 
TABLE II. Cr doping in AlP. The maximal photovoltaic efficiency is presented as well as the $T_{\mathrm{C}}$, exchange interaction and sizes of the gaps. $E_{1}, E_{2}$, $E_{g}$, and $E_{g}^{\prime}$ are the band edge separations as in Fig. 50. The efficiency is computed for operation at RT, for comparison with standard materials. $T_{\mathrm{C}}$ was below $160 \mathrm{~K}$ for the $\mathrm{Cr}$ concentration studied ( $8 \%$ cation).

\begin{tabular}{lcccc}
\hline \hline Configuration & Eff $(\%)$ & $T_{\mathrm{C}}(\mathrm{K})$ & $\Delta E_{\mathrm{FM}-\mathrm{AFM}}(\mathrm{eV})$ & \\
\hline AlP:Cr $\mathrm{Al}_{\mathrm{Al}}$ & 49.9 & 160 & -0.19 & \\
& $E_{1}(\mathrm{eV})$ & $E_{2}(\mathrm{eV})$ & $E_{g}(\mathrm{eV})$ & $E_{g}-E_{g}^{\prime}(\mathrm{eV})$ \\
& 1.33 & 0.52 & 2.43 & 0.08 \\
\hline \hline
\end{tabular}

efficiency. Moreover, looking at the full band structure, alloying with $\mathrm{Cr}$ produces an IB having direct transitions to both the $\mathrm{CB}$ and the VB. The compound is ferromagnetically stable and has an estimated $T_{\mathrm{C}} \sim 160 \mathrm{~K}$. The obtained calculated parameters are summarized in Table II. The ferromagnetic interaction between $\mathrm{Cr}$ atoms is long-ranged and particularly strong when the dopants are separated by $\mathrm{Al}$ atoms, due to anisotropic $p-d$ hybridisation as in GaAs doped with $\mathrm{Mn}^{207}$ Thus, a directed growth pattern could increase the ferromagnetic stability. The stability of the ferromagnetic solution is also increasing with concentration (see Fig. 54). At the same time, a doping level higher than $10 \%$ broadens the IB prohibitively for practical photovoltaic applications. $T_{\mathrm{C}}$ presented here corresponds well with those found in the literature ${ }^{186}$ and is high enough for practical demonstration of the effect here described. Indeed, alloys GaAlP:Cr have been produced that exhibits large $T_{\mathrm{C}}{ }^{208,209}$

Having defect levels or bands within the bandgap, a quite common feature is unlikely by itself to provide the expected solar cell efficiency enhancement, but under favourable conditions, a ferromagnetic implementation, or other scheme to prevent enhanced nonradiative recombination through the IB as shown in Fig. 4, could yield an efficiency benefit in practice. The dilute $\mathrm{Cr}$ doped AlP compound that has been shown to fulfil the criteria for optimal photovoltaic conversion may be one of them. With the rapid

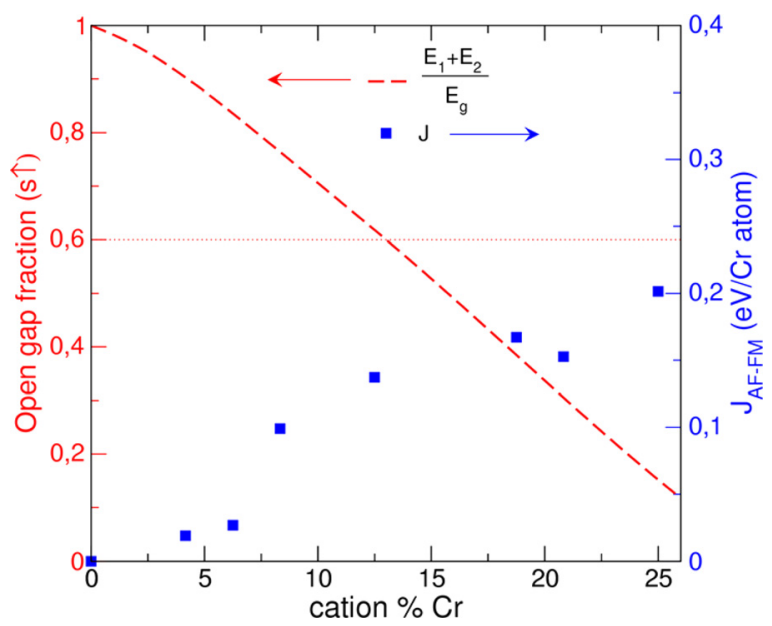

FIG. 54. Magnetic stability (squares, right hand scale) versus the open fraction of the gap (dashed line, left hand scale) as functions of the $\mathrm{Cr}$ concentration for compounds with randomly dispersed dopant atoms. The open gap fraction decreases with increasing dopant concentration. Adapted with permission from Olsson et al., Phys. Rev. Lett. 102, 227204 (2009). Copyright 2009 American Physical Society. progresses of semiconductors for both spintronic and VISUV LED's, suitable materials for high efficiency photovoltaics may be found and evaluated in the future.

\section{Spectroscopy measurements and techniques}

When standard solar cell measurements are performed on an IBSC, it is not immediately apparent from the solar cell $J-V$ characteristics whether the IBSC is operating as desired, or as a conventional two-band solar cell. Adding additional states to a high bandgap solar cell can increase the photocurrent and reduce the operating voltage, mimicking the $J-V$ result shown in Fig. 1. The extent to which the current is increased and the voltage reduced depends upon the density of those states, so detailed modelling is required to determine whether the interplay between current and voltage follows what would be expected for an IBSC, or a conventional two-band solar cell. However, spectroscopic tests can be used to discriminate true IBSC behaviour from conventional two-band solar cells. ${ }^{210}$ We first discuss two basic PL tests and then consider measurement of TSPA; photocurrent production, carrier absorption/recombination dynamics, and inter-subband photocurrent spectroscopy.

\section{Steady state emission from an IBSC}

A simple steady-state PL (or EL) measurement will normally reveal the lowest energy states in a conventional twoband solar cell. In an IBSC, additional strong emission peaks should be observed corresponding to the VB-IB transition, CB-IB transition, and VB-CB transition. The simulated PL is modeled using the generalised Planck equation, derived by Würfel. ${ }^{17}$ The PL intensities are simulated assuming detailed balance between absorbed and emitter photon fluxes. A simulated example is shown in Fig. 55, where an IBSC with energy thresholds at $1.96 \mathrm{eV}, 1.24 \mathrm{eV}$, and $0.72 \mathrm{eV}$ is pumped optically at an energy in-excess of $1.96 \mathrm{eV}\left(G_{\mathrm{VC}}\right)$, thereby photo-generating carriers in the $\mathrm{CB}$ only. The emission spectrum is calculated assuming only radiative transitions exist, therefore providing an indication of the spectral signature for an ideal IBSC material. ${ }^{210}$ The clear signature

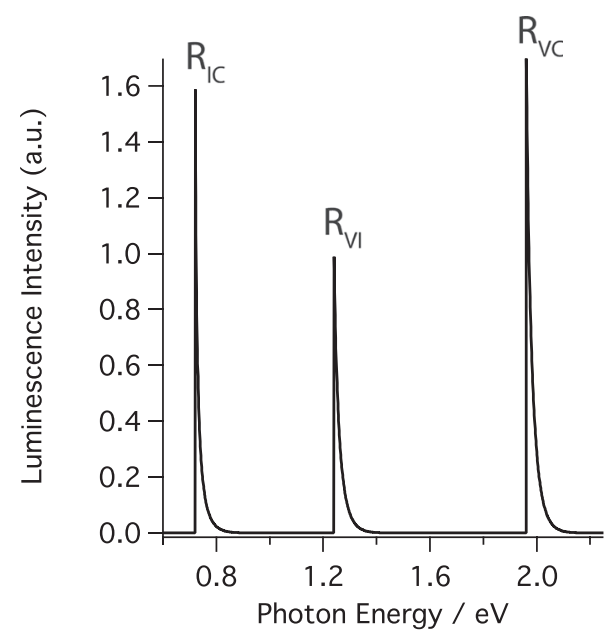

FIG. 55. Simulated PL from an ideal IBSC material, showing three strong and roughly equal PL peaks over an extremely broad photon energy range. 
of a radiatively efficient IBSC material is given by the roughly equal emission intensities from all three optical transitions. This arises when the recombination pathways, either directly to the VB, emitting one photon, or via the IB emitting two photons are roughly equal, as required for efficient operation. This behaviour is in stark contrast to a thermal electron population where the emission at $0.72 \mathrm{eV}$ would be approximately twenty orders of magnitude more intense than the emission at $1.96 \mathrm{eV}$.

A slightly more complex verification for the IBSC can be made if the two optical transitions $G_{\mathrm{VI}}$ and $G_{\mathrm{IC}}$ are excited, pumping carriers into the $\mathrm{CB}$ via the IB. Here, a functioning IBSC material will show up-conversion, namely, the emission of light at the sum of the two incident photon energies. A simulated example of the detailed behavior of an ideal IBSC is shown in Fig. 56, illustrating both strong up-conversion and PL from all optical transitions in the device. One advantage of this up-conversion experiment is that it can be performed on bare IBSC material, enabling its function to be confirmed before contacts are made.

\section{Two-step photon absorption (TSPA) photocurrent spectroscopy}

Once contacts are made to an IBSC material, a photocurrent can be measured. This has the virtue of enabling bias conditions other than open-circuit (under which the two tests above would be performed) and provides a more quantitative measure of the TSPA taking place in the device. Conventional monochromatic EQE spectroscopy cannot determine the gain in photocurrent via TSPA processes; instead an IR bias light is used to promote TSPA that induces a change in the EQE $(\triangle \mathrm{EQE})$.

$\triangle \mathrm{EQE}$ induced by inter-subband photocurrent under IR bias light illumination is described by the following equation:

$$
\Delta \mathrm{EQE}=\mathrm{EQE}_{w / \mathrm{IR}}-\mathrm{EQE}_{w / o \mathrm{IR}} .
$$

A typical continuous wave (CW) white-light IR biased $\triangle \mathrm{EQE}$ spectroscopy setup is shown schematically in Figs. 57(a) and 58(a). CW monochromatic light generated by a white-light source (Xenon or halogen lamp) and a

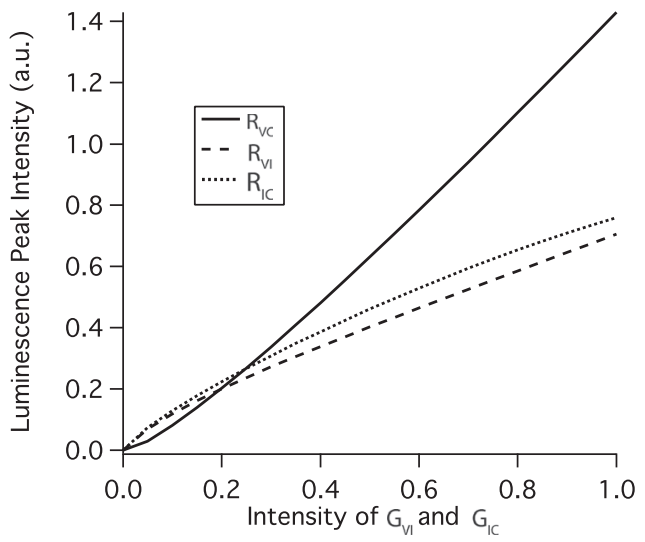

FIG. 56. Simulated up-converted PL $R_{\mathrm{VC}}$ from an ideal IBSC material while pumping $G_{\mathrm{VI}}$ and $G_{\mathrm{IC}}$.
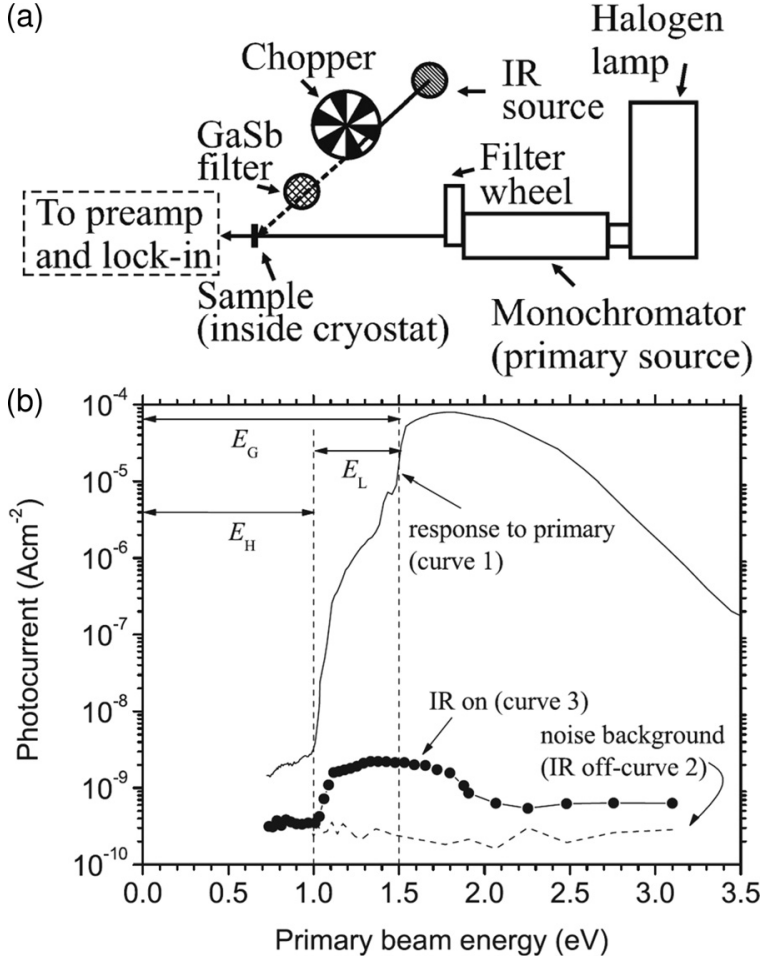

FIG. 57. (a) Schematic optical setup for low temperature white-light IR biased inter-subband photocurrent spectroscopy. The lock-in amplifier is synchronized with an optical chopper modulating CW IR bias light. (b) Photocurrent produced in a Si $\delta$-doped InAs/GaAs QDSC by the interband transition by monochromatic light (curve 1 ; at $V=-1.5 \mathrm{~V}, T=4.2 \mathrm{~K}$ ), noise background of lock-in detection (curve 2; at $V=0 \mathrm{~V}, T=36 \mathrm{~K}$ ), and intersubband transition by monochromatic and IR bias light (curve 3 at $V=0 \mathrm{~V}$, $T=36 \mathrm{~K})$. Reprinted with permission from Martí et al., Phys. Rev. Lett. 97, 247701 (2006). Copyright 2006 American Physical Society.

monochromator causes the first step interband transition and is used to control the quasi-Fermi level separation in the IBSC. With the additional white-light source, the IR bias light is produced by using a long pass filter. Typical intensity of the IR bias light is $\sim 10 \mathrm{~mW} / \mathrm{cm}^{2}$, when spectrally integrated across the IR part of the 1 sun illumination of AM $1.5 \mathrm{G}$ spectrum. In conventional EQE measurement apparatus, as shown in Fig. 58(a), the optical chopper is located at the $\mathrm{CW}$ monochromatic light side and the difference in $\mathrm{EQE}$ spectra with and without IR bias light is determined. In Fig. 57(a), the photocurrent intensity modulation by IR bias light is recorded by a lock-in amplifier and synchronized with an optical chopper placing at IR bias light path. The signal to noise ratio of inter-subband photocurrent can be significantly improved because the background interband photocurrent signal by $\mathrm{CW}$ monochromatic light (usually several orders of magnitude larger than inter-subband photocurrent) can be subtracted by the lock-in detection.

There have been a large number of reports on the gain in photocurrent via TSPA from an IBSC. The photocurrent gain via TSPA by applying IR bias light was first demonstrated by Martí et al. at low temperature $(36 \mathrm{~K})$ in a $\mathrm{Si} \delta$-doped InAs/GaAs QDSC as shown in Fig. 57(b). ${ }^{172}$ The changes in photocurrent under white-light IR bias irradiation were recorded by scanning the first step interband transition photon energy. This result has been confirmed by EQE 

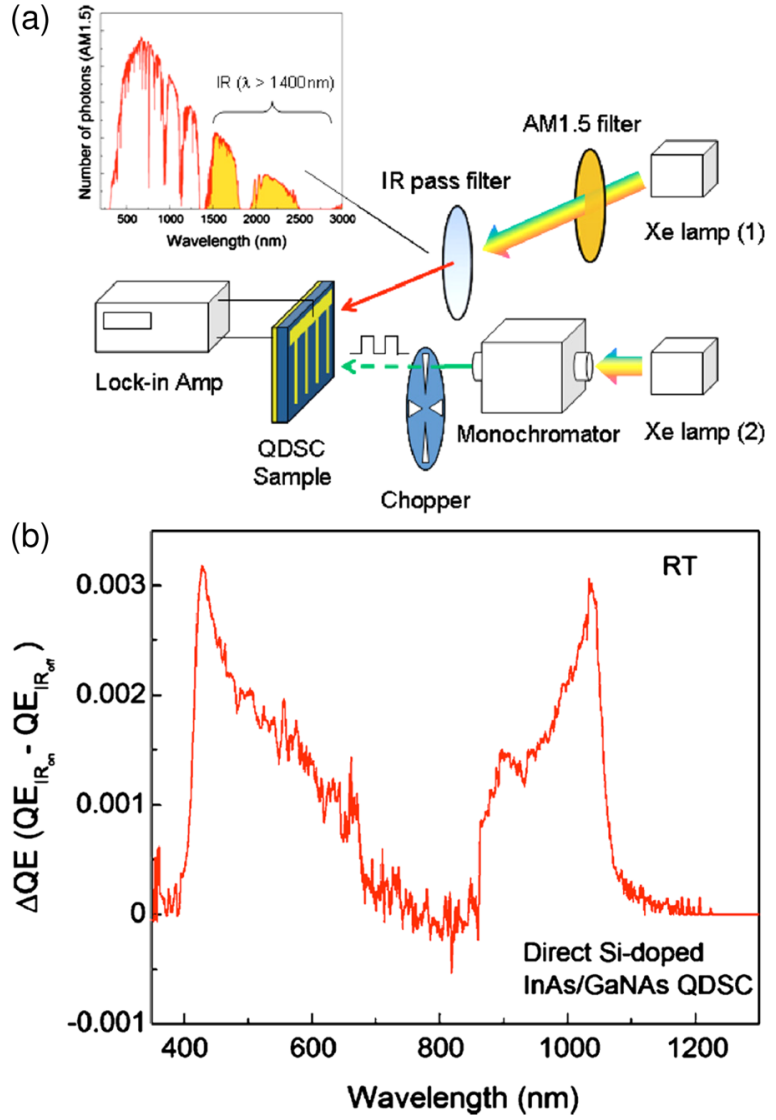

FIG. 58. (a) Schematic optical setup for IR part of 1 sun illumination (AM 1.5) biased inter-subband photocurrent spectroscopy. The difference of with and without IR bias light is determined. (b) $\triangle \mathrm{EQE}$ spectrum with and without IR illumination in a direct Si-doped InAs/GaNAs QDSC at RT. Adapted with permission from Okada et al., J. Appl. Phys. 109, 024301 (2011). Copyright 2011 AIP Publishing LLC.

enhancement at RT in InAs/GaAs based QDSCs. ${ }^{124,137,183,211,212}$ Figure 58(b) indicates the first demonstration at RT of $\triangle \mathrm{EQE}$ by inter-subband photocurrent. As described in Sec. III B, QDSCs have been widely studied to
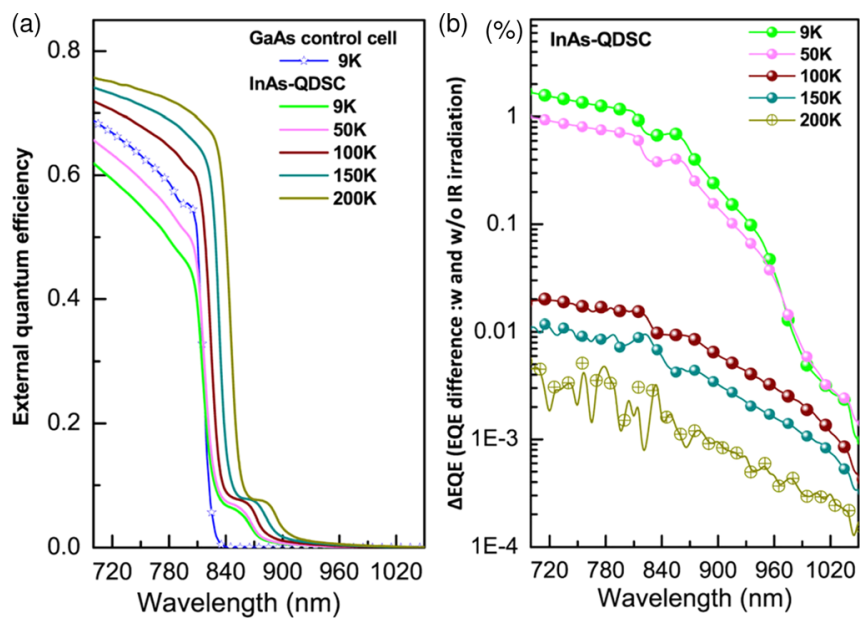

FIG. 59. (a) Low temperature EQE spectra of an InAs/GaAs QDSC and a GaAs control cell. (b) Low temperature $\triangle \mathrm{EQE}$ spectra of the QDSC difference of with and without CW IR white-light bias irradiation. Adapted with permission from Sogabe et al., Sci. Rep. 4, 4792 (2014). Copyright 2014 Nature Publishing Group. achieve IBSCs, and photocurrent production via TSPA is investigated by several groups. ${ }^{35,36,124,137,140,172,183,211-218}$ Photocurrent gain induced by TSPA in GaNAs(Sb) solar cells has been investigated ${ }^{85,95}$ together with ZeTeO. ${ }^{64,97-99}$ In addition, inter-subband photocurrent production is also confirmed in MQW solar cells. ${ }^{219-222}$

However, the enhancement of EQE at RT is very weak, typically below $1 \%$ gain, and it is partly due to fast thermal excitation of photo-carriers from the IB. As described in Sec. II, the IB must be sufficiently filled to support efficient TSPA. In conventional $\mathrm{In}_{0.5} \mathrm{Ga}_{0.5} \mathrm{As} / \mathrm{GaAs}$ QDSCs, significant IR response can be observed below $70 \mathrm{~K} .{ }^{213}$ Figure 59(a) shows temperature dependence of EQE spectra in an InAs/GaAs QDSC. ${ }^{183}$ Decreasing temperature suppresses the carrier collection efficiency for the QDSC above the GaAs bandgap energy and thermal excitation of photocarriers from QDs can be reduced. Figure 59(b) shows low temperature $\mathrm{CW}$ white-light IR biased $\triangle \mathrm{EQE}$ spectra and a dramatically enhanced EQE, of for more than $1 \%$, was obtained at $9 \mathrm{~K}$. To suppress the thermal excitation of photocarriers from the IB and enhance inter-subband photocurrent production, type-II GaSb QD, ${ }^{140}$ higher bandgap barrier materials (AlGaAs), ${ }^{36,217,223-226}$ type-II wide bandgap barrier (AlAsSb), ${ }^{218}$ or blocking layers ${ }^{64,85,95,98,99}$ have been investigated by TSPA photocurrent spectroscopy.

The dependence of the IB filling condition on photocurrent production via TSPA has been investigated by applying a DC bias voltage ${ }^{137,183,212,213}$ and intensity dependent interband photoexcitation. ${ }^{213,214,224}$ Field-assisted tunneling from IB is reduced by applying forward DC bias hence filling the $\mathrm{IB}$ and increasing and higher $\triangle \mathrm{EQE}$ is obtained. Larger forward bias application decreases $\triangle \mathrm{EQE}$ due to insufficient carrier collection through CB after TSPA. Figure 60 shows the photocurrent production via inter-subband transition as a function of interband photocurrent in an $\operatorname{In}_{0.5} \mathrm{Ga}_{0.5} \mathrm{As} / \mathrm{GaAs}$ QDSC at $20 \mathrm{~K}^{213}$ The interband and inter-subband photocurrent are obtained at -3.0 and $0.5 \mathrm{~V}$, respectively. Although increasing interband excitation intensity increases the QD

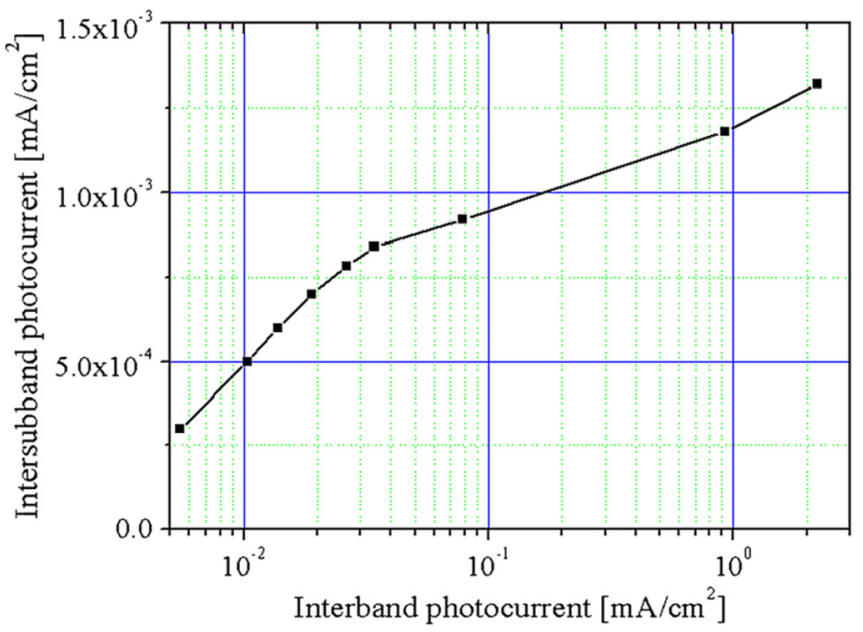

FIG. 60. Interband photocurrent dependence on inter-subband photocurrent in an $\mathrm{In}_{0.5} \mathrm{Ga}_{0.5} \mathrm{As} / \mathrm{GaAs}$ QDSC at $20 \mathrm{~K}$. The interband and inter-subband photocurrent are measured at applied bias at $-3.0 \mathrm{~V}$ and $0.5 \mathrm{~V}$, respectively. Reprinted with permission from Jolley et al., Prog. Photovoltaics: Res. Appl. 21, 736 (2013). Copyright 2013 John Wiley and Sons, Ltd. 
electron occupancy, hence leading to recombination being favored over absorption and saturating the inter-subband transition. In this regime, increasing the interband excitation intensity does not increase the inter-subband photocurrent production significantly. ${ }^{213,214}$

\section{Carrier dynamics of inter-subband transition in InAs/GaAs QDs}

Self-assembled InAs QDs were grown on a $\mathrm{GaAs}(001)$ substrate using MBE. The average QD height was $5 \mathrm{~nm}$, and the base size was $20 \mathrm{~nm}$. The QD density was approximately $4 \times 10^{10} \mathrm{~cm}^{-2}$. Three QD layers were stacked with a spacer layer of GaAs with the thickness of $50 \mathrm{~nm}$. This spacer layer is thick enough to separate each QD layer, and thus the electronic coupling along the stacked direction is negligible. In this experiment, a one-dimensional photonic cavity structure was introduced to enhance the inter-subband transition strength (see Fig. 61). The three QD layers were inserted into the center position of a cavity behaving the anti-node of the electromagnetic field to enhance the interaction between the QD and light. The cavity structure is comprised of a couple of distributed Bragg reflector (DBR) mirrors of GaAs and AlAs. The periods of the top and bottom mirrors were 5 and 9 , respectively. A normal reflectivity spectrum of the cavity structure measured at RT demonstrates the resonant wavelength of $1300 \mathrm{~nm}$ and the measured $\mathrm{Q}$ value of this cavity was 77 .

To characterize carrier dynamics in the intermediate state, two-color photo-excitation spectroscopy is developed as illustrated in Fig. 62 using a near-IR streak-camera system with the temporal resolution of $20 \mathrm{ps}^{227} \mathrm{~A} \mathrm{CW}$ laser light with the wavelength of $659 \mathrm{~nm}$ irradiated the sample to create carriers, which controls the quasi-Fermi level. And then, the intermediate state was excited by $1300 \mathrm{~nm}$ pulsed laser light (see Fig. 61). The IR pulsed light source used was generated by an optical parametric oscillator (OPO) excited by a mode-locked Ti:Sapphire laser. The pulse duration was approximately $200 \mathrm{fs}$ and the repetition rate was $80 \mathrm{MHz}$. In this study, interband TR-PL of QD was observed. The PL intensity corresponds to the electron density in the

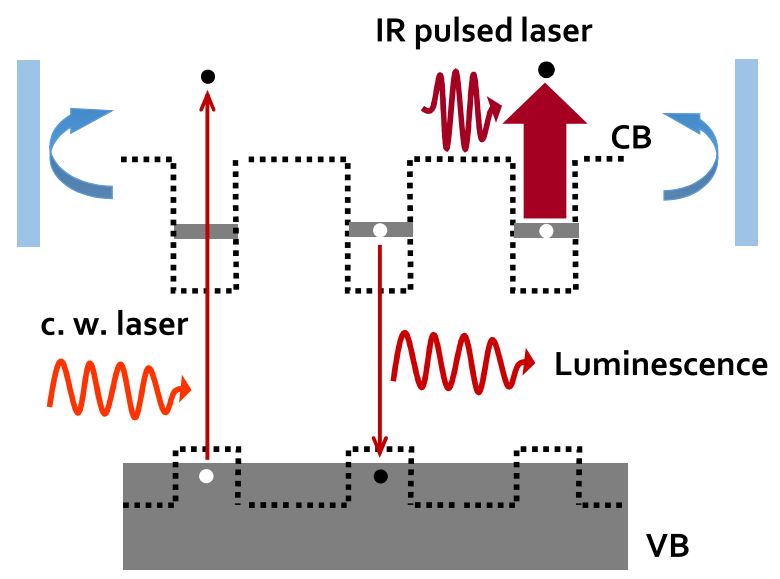

FIG. 61. InAs/GaAs QD layers with a one-dimensional cavity structure. Two-color photo-excitations done for characterizing carrier dynamics are illustrated.

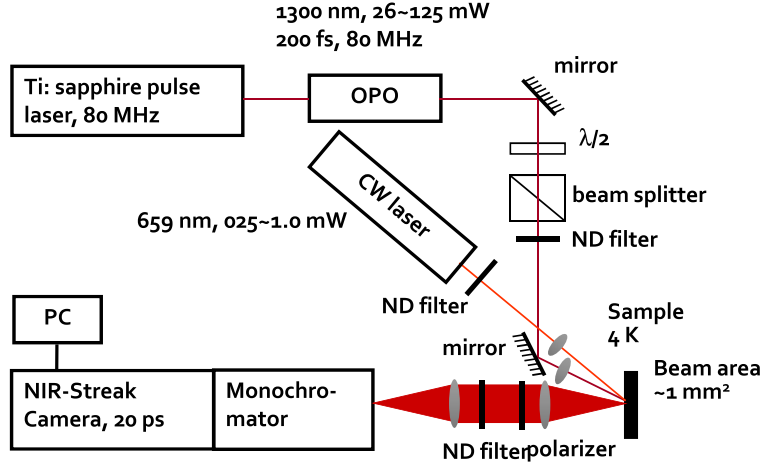

FIG. 62. Schematic illustration of experimental setup of two-color photo-excitation TR-PL spectroscopy.

intermediate state. Therefore, the pulse modulated electron density can be observed by monitoring the PL intensity.

Figure 63 shows PL spectra measured at various excitation powers. The measurement was carried out at $17 \mathrm{~K}$, and the excitation energy was $1.88 \mathrm{eV}$ which is greater than the GaAs bandgap. With the increase in the excitation power, PL signals from the excited states become strong significantly. The peak wavelength of the PL is approximately $1140 \mathrm{~nm}$, which is shorter than the pulse excitation wavelength of $1300 \mathrm{~nm}$. Therefore, the pulse laser light is transparent for the interband transition and is enabled to excite electrons in the QD state acting as the intermediate state.

A typical spectrum of the two-color photo-excitation spectroscopy is shown in Fig. 64. ${ }^{227,228}$ The PL intensity decreased immediately after the excitation. This indicates electrons in the intermediate state were pumped out by the IR-pulse excitation. This is a key process in the IBSC. It is noted that this intensity reduction was followed by a gain. This gain is caused by extra carrier injection from the barrier layer. With the increase in the IR-pulse excitation power, the signal amplitude increases almost linearly, and then, shows a saturation. This saturation arises from a complete excitation of electrons created by the CW excitation, in other words, saturable absorption occurs. ${ }^{184,229}$

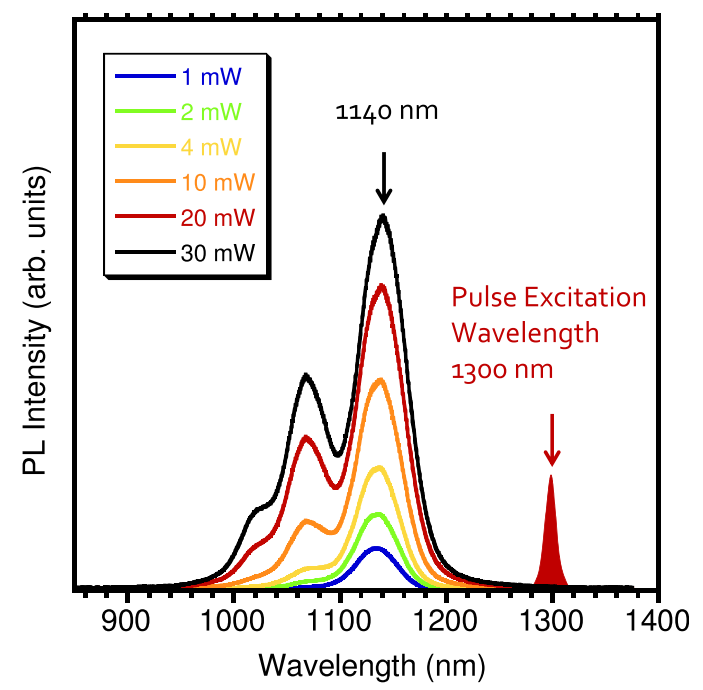

FIG. 63. Excitation-power dependence of PL spectra measured at $17 \mathrm{~K}$. The excitation energy was $1.88 \mathrm{eV}$. 


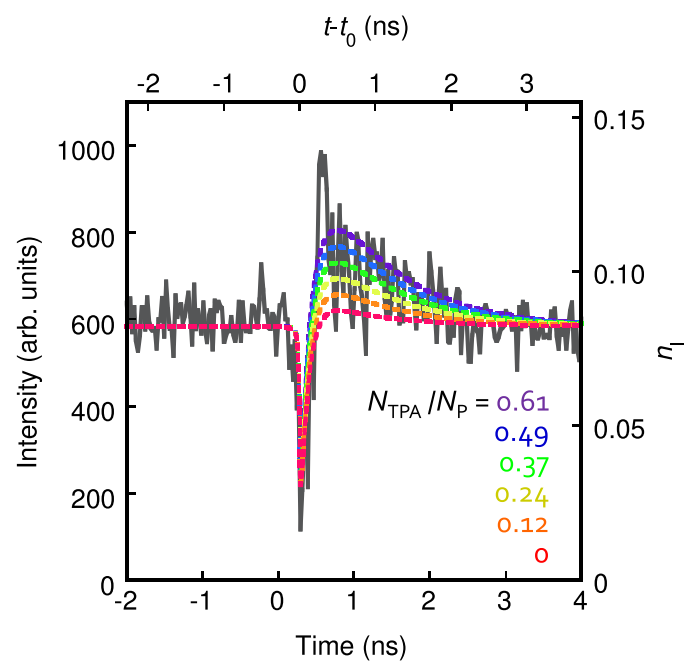

FIG. 64. Typical two-color photoexcitation spectroscopy signal and theoretical calculations obtained by varying contribution of TPA.

Figure 65 shows the pulse-excitation power dependence of the maximum PL intensity reduction. Squares, circles, and triangles indicate the results for the $\mathrm{CW}$-excitation powers of $0.25,1.0$, and $1.5 \mathrm{~mW}$, respectively. The PL-intensity reduction strongly depends on the $\mathrm{CW}$-excitation power; the low $\mathrm{CW}$-excitation power considerably reduces the PL intensity at low pulse-excitation power.

In order to understand the observed $\mathrm{CW}$ - and pulseexcitation power-dependent PL dynamics, the phenomenological rate equations are developed by taking into account the effect of the saturable absorption for the bound-to-continuum inter-subband transition. ${ }^{184,227}$ Figure 66 shows a model for interpreting the observed results. The $\mathrm{CW}$ laser excites the VB electrons to the $\mathrm{CB}$ at the rate $G$. The populated electron density in the $\mathrm{CB}$ is $n_{\mathrm{C}}$. The excited electrons relax to the intermediate state or the $\mathrm{VB}$ with time constants of $\tau_{\mathrm{IC}}$ and $\tau_{\mathrm{C}}$, respectively. The electron density of the intermediate state is $n_{\mathrm{I}}$, which is proportional to the PL intensity. Electrons in the intermediate state recombine in radiative and nonradiative processes with a time constant of $\tau_{\mathrm{I}}$. The IR

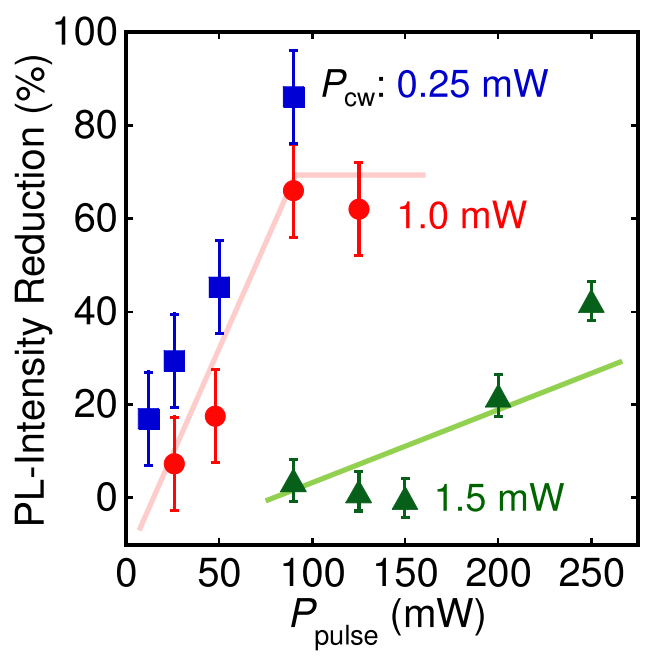

FIG. 65. IR-pulse-excitation-power dependence of the two-color photo-excitation spectroscopy signal as a function various interband CW-excitation intensity.

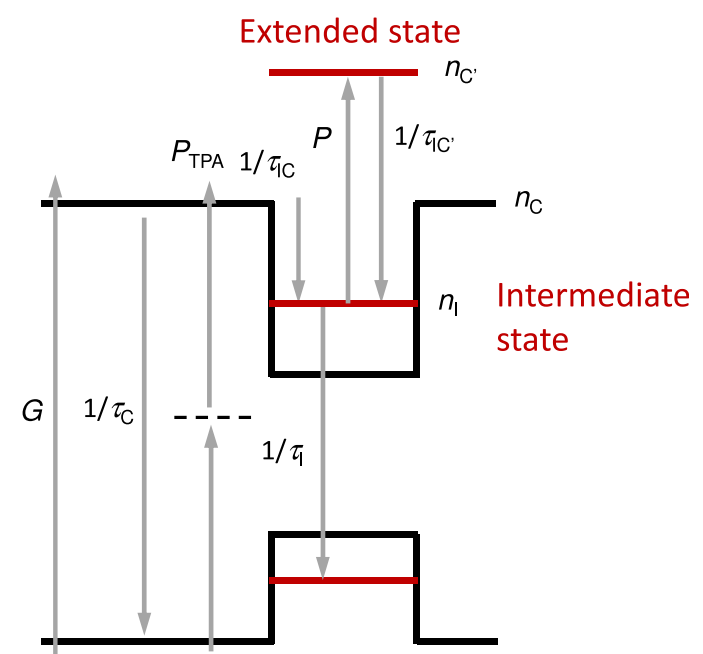

FIG. 66. Three-level model used in the rate equation analysis.

laser pulse excites the intermediate state electrons to the CB. The density of the electrons that are excited by the IR laser pulse in the $\mathrm{CB}$ is $n_{\mathrm{C}^{\prime}}$, which relaxes to the intermediate state with a time constant of $\tau_{\mathrm{IC}^{\prime}}$. The excitation rate by the IR laser is expected to be proportional to the laser shape represented by a $\operatorname{sech}^{2}$-shaped function. The number of excited electrons per pulse is $N_{\mathrm{P}}$. Using these parameters, the following phenomenological rate equations can be derived:

$$
\begin{gathered}
\frac{\partial n_{\mathrm{C}}}{\partial t}=G-\frac{n_{\mathrm{C}}}{\tau_{\mathrm{IC}}}\left(1-\frac{n_{\mathrm{I}}}{2}\right)-\frac{n_{\mathrm{C}}}{\tau_{\mathrm{C}}}+P_{\mathrm{TPA}}, \\
\frac{\partial n_{\mathrm{C}^{\prime}}}{\partial t}=-\frac{n_{\mathrm{C}^{\prime}}}{\tau_{\mathrm{IC}}}\left(1-\frac{n_{\mathrm{I}}}{2}\right)+P, \\
\frac{\partial n_{\mathrm{I}}}{\partial t}=\frac{n_{\mathrm{C}}}{\tau_{\mathrm{IC}}}\left(1-\frac{n_{\mathrm{I}}}{2}\right)-\frac{n_{\mathrm{C}^{\prime}}}{\tau_{\mathrm{IC}}}\left(1-\frac{n_{\mathrm{I}}}{2}\right)-\frac{n_{\mathrm{I}}}{\tau_{\mathrm{I}}}-P, \\
P_{(\mathrm{TPA})}=\frac{N_{\mathrm{P}(\mathrm{TPA})}}{2 \Delta} \operatorname{sech}^{2}\left(\frac{t-t_{0}}{\Delta}\right) .
\end{gathered}
$$

These equations include the Pauli blocking factor $1-n_{\mathrm{I}} / 2$ for the intermediate state and nonlinear two-photon absorption (TPA) process. In the present study, the effect of saturable absorption on the bound-to-continuum inter-subband transition was taken into account when $N_{\mathrm{P}}$ is larger than the $n_{\mathrm{I}}$ at the steady-state. The recombination time in the GaAs $\mathrm{CB}, \tau_{\mathrm{C}}$, was assumed to be $3.3 \mathrm{~ns}$, which corresponds to the radiative lifetime of free excitons in GaAs. ${ }^{230}$ The time constants were estimated to be $\tau_{\mathrm{IC}}=0.3 \mathrm{~ns}$ and $\tau_{\mathrm{I}}=0.9 \mathrm{~ns}$, from the TR-PL of the interband transition. ${ }^{227}$ Using these values, the PL response is calculated according to the temporal dependence of $n_{\mathrm{I}}$ which is broadened by convolution with a Lorentzian with a full width at half maximum of 20 ps which is the resolution of the streak camera system.

Typical calculated responses of PL intensity created by a pulse excitation at $G=0.1 \mathrm{~ns}^{-1}$ with varied $N_{\mathrm{TPA}} / N_{\mathrm{P}}$ drawn by dashed lines in Fig. 64. $N_{\mathrm{P}}$ is assumed to be equal to the steady-state value of $n_{\mathrm{I}}\left(n_{\mathrm{I}, \mathrm{SS}}\right)$. The observed PL response is well reproduced by this calculation. ${ }^{227}$ The electron density in the intermediate state decreases immediately after the pulse excitation. This reduction rapidly recovers 
because of rapid carrier injection from the adjacent GaAs layers. The dip structure is followed by a gain with a slow temporal evolution. This gain feature is attributed to extra carrier injection from the GaAs barrier region into QDs, which arises from the TPA process caused by the intense IR laser pulse excitation. The injected electrons recombine with holes with the lifetime of $\tau_{\mathrm{I}}$. Thus, the $\tau_{\mathrm{I}}$ limits the temporal evolution of the gain.

The pulse-excitation power is proportional to $N_{\mathrm{P}}+2 N_{\mathrm{TPA}}$, whereas the inter-subband transition rate is proportional to $n_{\mathrm{I}, \mathrm{SS}}$. Therefore, the pulse-excitation power is proportional to $\left(N_{\mathrm{P}}+2 N_{\mathrm{TPA}}\right) / n_{\mathrm{I}, \mathrm{SS}}$ when the incident photon number of the pulsed light is much larger than $N_{\mathrm{P}}+2 N_{\mathrm{TPA}}$. Figure 67 shows the calculated pulse-excitation power dependence of the maximum PL-intensity reduction at the $N_{\text {TPA }} / N_{\mathrm{P}}^{2}$ of $3 .{ }^{184}$ The maximum PL-intensity reduction decreases with an increase in $G$, which results from the rapid carrier relaxation from GaAs into QDs. On the other hand, a saturation of the maximum PL-intensity reduction is obvious with an increase in the pulse-excitation power when $G$ is smaller than $0.1 \mathrm{~ns}^{-1}$. This feature is caused by the saturable absorption for the bound-to-continuum inter-subband transition. The calculated PL-intensity reduction gradually decreases with the increase in $G$ at the $\left(N_{\mathrm{P}}+2 N_{\text {TPA }}\right) / n_{\text {I.SS }}$ of less than 1 . The calculated pulse-excitation power dependence of the maximum PL-intensity reduction shown in Fig. 67 moderately reproduces the observed results in Fig. 65. According to this analysis, the absorption coefficient for the bound-to-continuum transition has been estimated to be approximately $200 \mathrm{~cm}^{-1}$. Here, the intermediate state was approximately $4 \%$ filled. Therefore, the absorption coefficient is estimated to be approximately $5000 \mathrm{~cm}^{-1}$ in case the intermediate state is completely filled. According to the enhancement factor of 8 for the PL-intensity reduction in the cavity structure, ${ }^{227}$ the absorption coefficient without the cavity structure can be roughly estimated to be approximately $600 \mathrm{~cm}^{-1}$ at the completely filled condition. This absorption coefficient moderately agrees with the value recently calculated by Luque et $a .^{231}$ These results demonstrated that the inter-subband transition is strong enough to provide a quick path to the $\mathrm{CB}$ for electrons pumped from the VB to the IB.

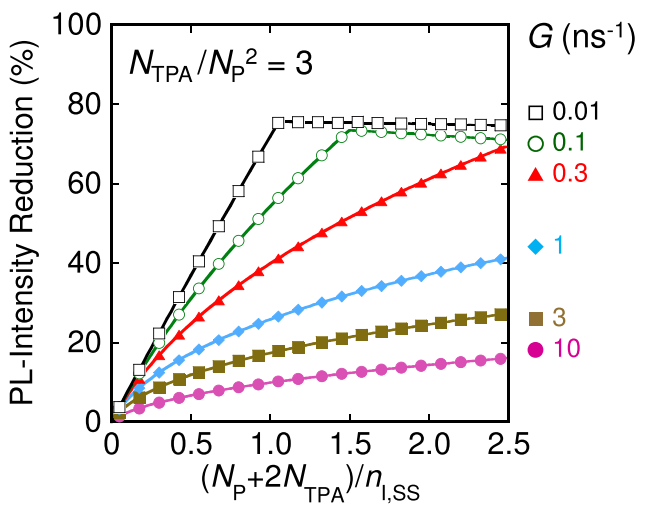

FIG. 67. Pulse-excitation power dependence of the two-color photo-excitation spectroscopy signal calculated for various $\mathrm{CW}$-excitation rate $G$.
The strength of the bound-to-continuum transition is crucial to achieve efficient TSPA and energy conversion efficiency above Shockley-Queisser limit. Detailed balance calculations ${ }^{177,232}$ revealed $\alpha \sim 1000 \mathrm{~cm}^{-1}$ which is necessary to achieve above $40 \%$ efficiency in thermodynamic limit. Absorption coefficients of IB-CB inter-subband transition, $\alpha_{\mathrm{IC}}$, in closely packed QD-SLs have been determined by $k \cdot p$ calculations. Luque et al. ${ }^{231}$ evaluated $\alpha_{\mathrm{IC}} \sim 100 \mathrm{~cm}^{-1}$ in a vertically coupled QD-SL with half-filled IB, $f_{\mathrm{I}}=0.5$. Tomić et al. ${ }^{233}$ determined $\alpha_{\mathrm{IC}} \sim 100 \mathrm{~cm}^{-1}$ in a horizontally coupled QD-SL with $f_{\mathrm{I}}=1.0$. The localized nature of QD quantized states reduces spacial overlap integrals of wavefunctions between continuum states, which are sparse in threedimensional volume. Therefore, the $\alpha_{\mathrm{IC}}$ is usually smaller than the ones for interband transitions. However, the selection rules of dipole transitions in zero-dimensional quantized states are allowed to excite IB electrons optically to CB unlike in two-dimensional QWs. Furthermore, partially delocalized wavefunctions by forming QD-SL would be preferable to realize the larger $\alpha_{\mathrm{IC}}$ than intermediate state in isolated QDs, e.g., low in-plane density or thick spacer layers. The moderate $\alpha_{\text {IC }}$ in currently available QDSCs will be improved by fabricating higher in-plane QD density as described in Sec. IIIB 1 together with utilizing lightmanagement schemes. ${ }^{234,235}$

\section{Carrier recombination process in InAs/GaAs QD-IBSCs}

An IB placed in the bandgap of a host semiconductor plays a key role in extra photo-absorption. If photoexcitation provides a quick pass to the $\mathrm{CB}$ for electrons pumped from the VB to the IB, extra photocurrent is generated. The optical transition probability from the IB to the $\mathrm{CB}$ is proportional to the electron density in the IB. Therefore, electron escape from the IB reduces the extra photocurrent generation in IBSCs and lowers the $V_{\mathrm{OC}}$. Kasamatsu et al. studied the effects of the internal electric field on the carrier wavefunctions and tunneling-assisted carrier escape in InAs/ GaAs QD-IBSCs using TR-PL measurements. ${ }^{236}$ To focus on the effects of the electric field, they studied the optical responses at low temperature which provides a condition the thermal carrier escape process can be ignored.

InAs/GaAs QD-IBSC structures were grown on $n^{+}$$\mathrm{GaAs}(001)$ substrates using solid-source MBE. An undoped nine-stacked InAs/GaAs QD layers was grown at $480^{\circ} \mathrm{C}$ on a buffer layer of $i$-GaAs( $(1) / n$-GaAs $/ n^{+}$-GaAs grown at $550{ }^{\circ} \mathrm{C}$. The nominal thickness of the deposited InAs was $0.61 \mathrm{~nm}(2.0 \mathrm{ML})$, and the $\mathrm{As}_{2}$ flux during the QD growth was $1.3 \times 10^{-3} \mathrm{~Pa}$. The GaAs spacer layer thickness was $5.1 \mathrm{~nm}(18 \mathrm{ML})$. QDs were closely stacked along the growth direction so that the electronic states in the stacked QDs couple with each other. ${ }^{237,238}$ Subsequently, $p^{+}$-GaAs $/ p$ $\mathrm{AlGaAs} / p-\mathrm{GaAs} / i-\mathrm{GaAs}(2)$ layers were deposited on the stacked QD layers. The $p$-layers were deposited at $500^{\circ} \mathrm{C}$ and the $i$-GaAs layer was at $480^{\circ} \mathrm{C}$. The built-in electric field in the intrinsic layer was controlled by changing the thicknesses of the $i-\operatorname{GaAs}(1)$ and $i-\operatorname{GaAs}(2)$ layers. The expected internal electric fields applied to the QDs were 46 and 
$193 \mathrm{kV} / \mathrm{cm}$ when the total intrinsic layer thicknesses were 299.3 and $69.8 \mathrm{~nm}$, respectively. For a reference sample, nine-stacked QDs were grown on an undoped $\mathrm{GaAs}(001)$ substrate. The capping layer thickness of the GaAs in the reference sample was $100 \mathrm{~nm}$. Although the built-in internal electric field of this reference sample was not zero in a strict sense, it is referred as a sample with a very small internal electric field.

PL measurements were conducted through excitation using a laser diode with a wavelength of $659 \mathrm{~nm}$. TR-PL measurements were performed by using a near-IR streak camera system with a temporal resolution of $20 \mathrm{ps}$. The light source used was a mode-locked Ti:Sapphire pulsed laser with wavelengths of 800 and $900 \mathrm{~nm}$. The repetition rate was $4 \mathrm{MHz}$. These experiments were conducted under a shortcircuit condition to avoid photoinduced changes in the internal electric field.

Figure 68 shows the PL spectra measured at $16 \mathrm{~K}$. As the electric field increased, the PL peak wavelength exhibited an obvious redshift, and the PL intensity decreases dramatically. These are typical features of the quantumconfined Stark effect (QCSE). The in-plane linear polarization characteristics of the PL show a dramatic change with the electric field. The QDSC at $46 \mathrm{kV} / \mathrm{cm}$ exhibits obvious linear polarization anisotropy. The [110]-polarization component is stronger than the [110] component. This is direct evidence of VB mixing in stacked QDs where the electronic states are coupled electronically along the stacking direction, ${ }^{237,238}$ and therefore, a SL structure is formed. This anisotropy originally comes from the anisotropic QD shape and the piezoelectricity caused by the strain field. ${ }^{237,239}$ Conversely, the QDSC at $193 \mathrm{kV} / \mathrm{cm}$ does not show such polarization anisotropy. This indicates that electronic coupling along the stacking direction becomes weak under the strong electric field. Therefore, the electronic states are localized in each QD. This strong vertical confinement diminishes the anisotropy.

Figure 68 also displays the detection wavelength dependence of the PL decay profile measured at $3 \mathrm{~K}$. The reference sample shows a slow single exponential decay and clear detection wavelength dependence. The PL decay time was approximately $2 \mathrm{~ns}$. Since the decay time of conventional single-layer InAs/GaAs QDs is approximately $1 \mathrm{~ns},{ }^{170,227}$ the slow decay is attributed to delocalization of the exciton wavefunction in the stacked, coupled QDs. The detection wavelength dependence indicates spatial carrier transfer along the stacking direction, which has never been observed in single-layer QDs. On the other hand, the QDSC at $46 \mathrm{kV} / \mathrm{cm}$ shows a double exponential decay. The rapid initial decay is slightly shorter than $2 \mathrm{~ns}$, and the following slow decay becomes longer than $2 \mathrm{~ns}$. The internal electric field spatially separates electrons and holes toward the opposite directions along the stacked QDs. The detection wavelength dependence of this slow decay component indicates that the electronic states still extend in the SL under this internal electric field.

Conversely, at an extremely large internal electric field, a quite different PL decay profile is observed as shown in Fig. 68. The decay was very fast and can be described by double exponential components of $\tau_{1}$ and $\tau_{2}\left(\tau_{1}<\tau_{2}\right)$.
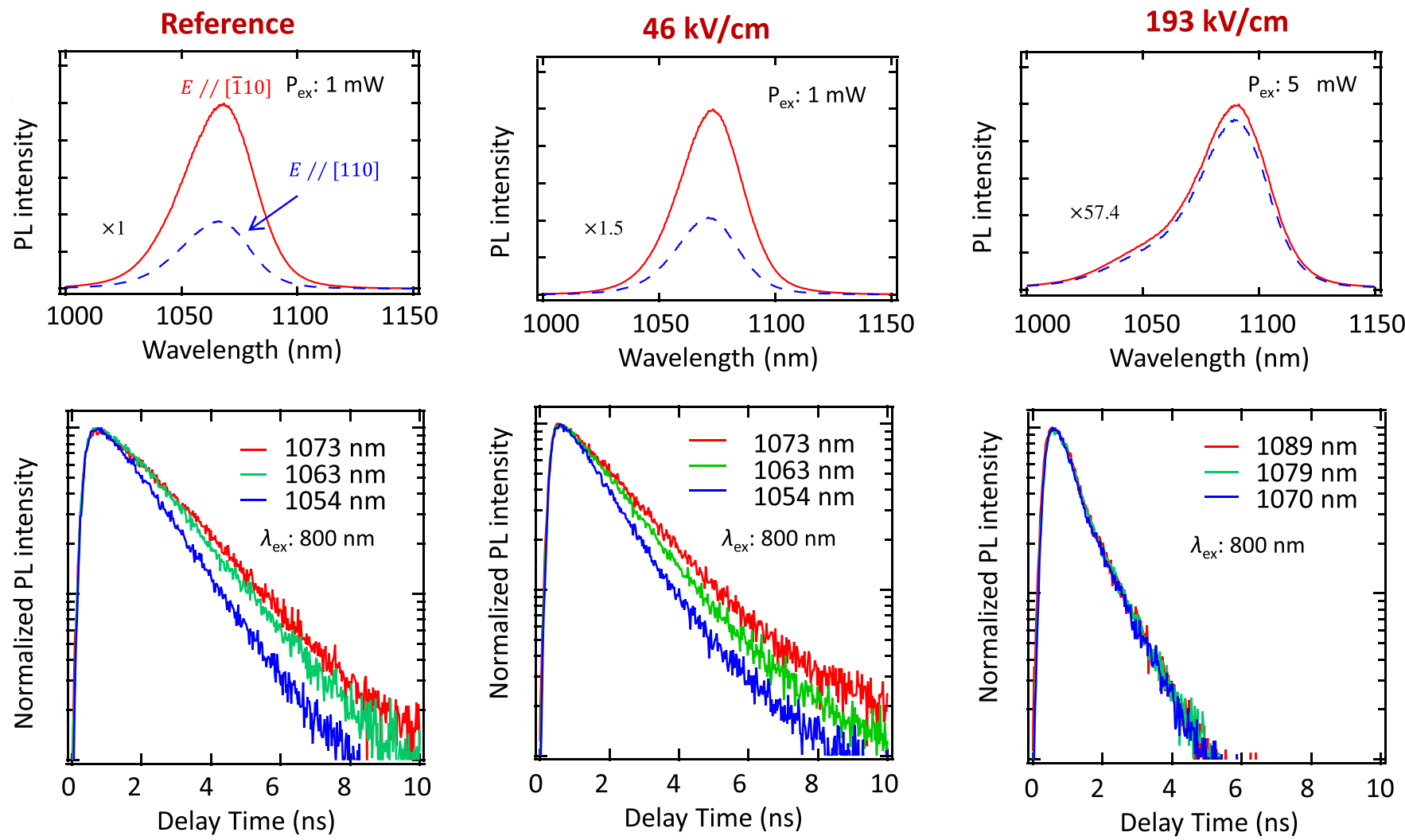

FIG. 68. In-plane linear PL-polarization anisotropy measured at $16 \mathrm{~K}$ and detection-wavelength dependence of PL decay profile at $3 \mathrm{~K}$ for a reference QDs and QDSCs at 46 and $193 \mathrm{kV} / \mathrm{cm}$ internal electric field. 
Besides, the detection wavelength dependence of the PL decay disappeared, which indicates that the field-induced decoupled energy states in QDs prohibit the spatial carrier transfer at the low temperature. The strong electric field causes substantial QCSE for the PL such as the remarkable intensity reduction and the large redshift. The recombination rate of electrons and holes should become greater in the decoupled QDs. The decay time $\tau_{2}$ was $1.09 \mathrm{~ns}$, which is close to a typical PL decay time in conventional single-layer QDs. It is noted that the hole mass of the fundamental state becomes large owing to the electric-field-induced decoupling of the electronic states in the stacked QDs. The hole quantization in the decoupled QDs becomes weaker than the electron's one. Besides, the electric field lowers the confining potential barrier. Therefore, the strong electric field can easily extract electrons from the QDs; in other words, tunneling-assisted electron escape occurs easily. The electron escape rapidly reduces the PL intensity with a time constant $\tau_{1}$ of $0.46 \mathrm{~ns}$. This short lifetime in the high electric field will prevent photo-excitation of electrons in the IB in QDs, and the $V_{\mathrm{OC}}$ of this QDSC eventually becomes small.

Efficient TSPA in IBSCs is achieved by suppressing both the tunneling and thermal electron escape processes. According to this work, tunneling electron escape turned out to be suppressed at the built-in electric field of $46 \mathrm{kV} / \mathrm{cm}$ which still maintains electronic coupling in stacked QDs.

\section{Spectrally resolved inter-subband photocurrent spectroscopy}

Tamaki et al. established optical characterization technique to investigate inter-subband transition photocurrent spectra by utilizing intense mid-IR pulsed laser as the wavelength tunable bias light source. ${ }^{214,215}$ Low temperature midIR pulse biased $\triangle \mathrm{EQE}$ spectroscopy setup is schematically shown in Fig. 69. Visible to near-IR CW monochromatic light, which causes the first step interband transition, is generated by a halogen lamp and a diffractive monochromator. Photocurrent spectra were normalized by the $\mathrm{CW}$ monochromatic light photon flux density to calculate EQE. The typical photon flux density is about $1.0 \times 10^{14}$ photons $\mathrm{cm}^{-2} \mathrm{~s}^{-1}$. Second harmonics of output pulses from a diode direct pumped regenerative amplifier (Light Conversion Ltd., PHAROS) with the repetition rate of $200 \mathrm{kHz}$ were used as

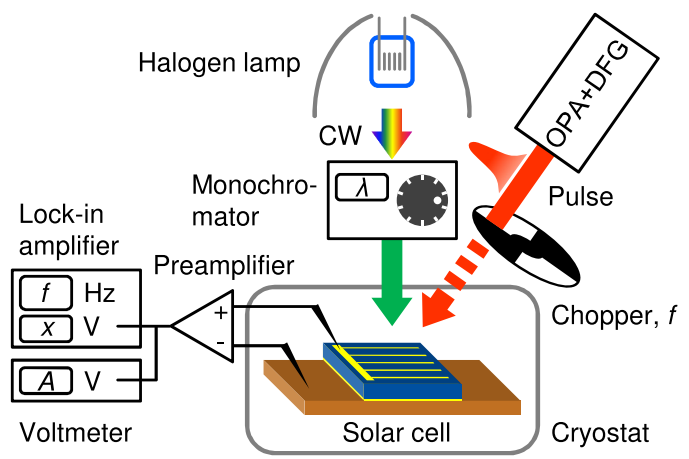

FIG. 69. Schematic representation of the IR pulse biased $\triangle \mathrm{EQE}$ spectroscopy setup. The OPA and DFG can generate intense wavelength tunable IR bias pulse. the pump for an optical parametric amplifier (OPA). Outputs from the OPA and difference frequency generation (DFG) were used as wavelength tunable intense IR bias light source, which triggers the second step inter-subband transition. The photon flux density of IR pulses was kept at $2.1 \times 10^{13}$ photons $\mathrm{cm}^{-2}$ per pulse, i.e., $4.2 \times 10^{18}$ photons $\mathrm{cm}^{-2} \mathrm{~s}^{-1}$, which is 40 times higher than that of long pass filtered $(>1.4 \mu \mathrm{m})$ spectrally integrated AM $1.5 \mathrm{G}$ of 1 sun illumination. The change in photocurrent under IR bias irradiation was detected to obtain $\triangle \mathrm{EQE}$ by a lock-in amplifier synchronized with an optical chopper modulating the IR pulse trains. To resolve both the first and second step transitions in TSPA, two-types of photon energy scanning experiments were performed. For the first step interband transitions, mid-IR bias photon energy was fixed and CW monochromatic light one was scanned. As for the second step inter-subband transitions, CW monochromatic photon energy was fixed and mid-IR bias photon energy was changed while fixing the mid-IR pulse photon flux density as described above.

Figure 70(a) resolves the second step inter-subband transitions by changing the photon energy of mid-IR pulses, whereas in Fig. 70(b), the first step interband transition photon energy is scanned while fixing the inter-subband transition photon energy. ${ }^{214,215}$ In Fig. 70(a), the inter-subband transition spectra by exciting $\mathrm{GaAs}(1.70 \mathrm{eV}$, blue) and wetting layers $(1.45 \mathrm{eV}$, green) exhibit a peak centered at
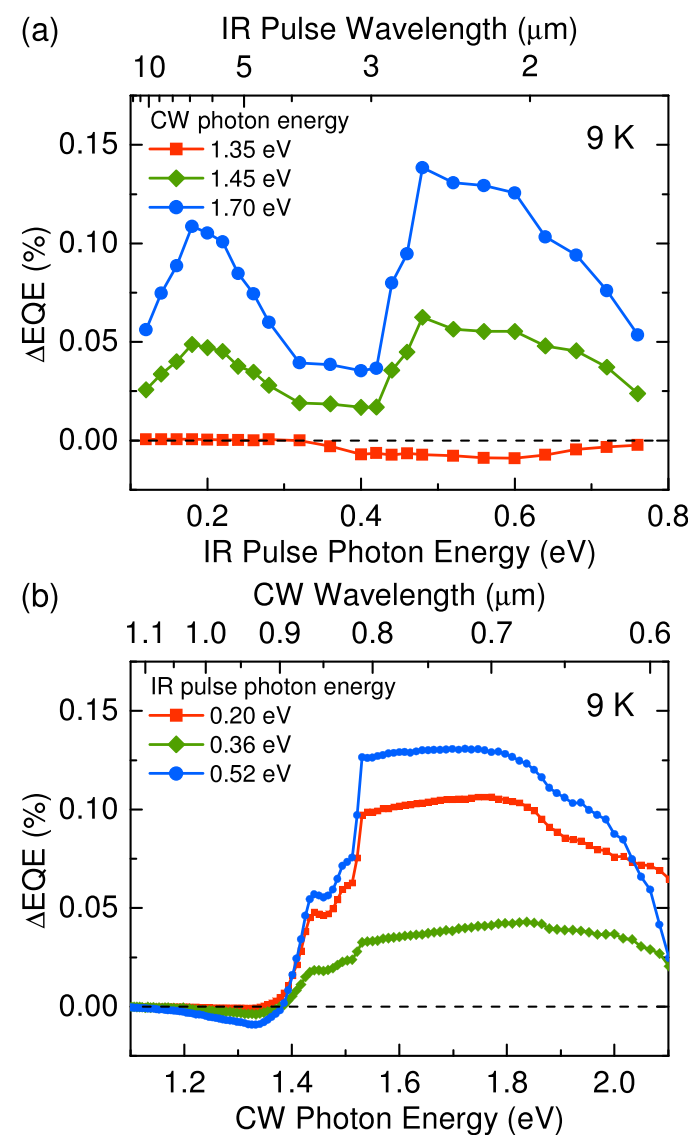

FIG. 70. $\triangle E Q E$ spectra by scanning (a) the second step inter-subband transition and (b) the first step interband transition of TSPA in an $\mathrm{In}_{0.4} \mathrm{Ga}_{0.6} \mathrm{As} /$ GaAs QDSC at $9 \mathrm{~K}$. Reprinted with permission from Tamaki et al., Appl. Phys. Lett. 105, 073118 (2014). Copyright 2014 AIP Publishing LLC. 
$0.20 \mathrm{eV}$ and a band above $0.42 \mathrm{eV}$. On the other hand, resonant excitation of QD ground states $(1.35 \mathrm{eV}$, red) results in EQE reduction by irradiating above $0.36 \mathrm{eV}$ mid-IR bias light. The $\triangle E Q E$ spectra at 1.45 and $1.70 \mathrm{eV}$ in Fig. 70(a) are similar. Therefore, the mid-IR bias light is exciting photo-carriers in the same initial states, QD ground states. The lower energy peak in Fig. 70(a) appears at larger photon energy than the total band offset between the QDs and GaAs $\mathrm{CB}(0.16 \mathrm{eV})$, indicating inter-subband transitions to the bound states above the GaAs CB edge, so-called "virtual bound states." 240 The higher energy band in Fig. 70(a) can be assigned as bound-to-continuum transitions to GaAs CB. The $\triangle \mathrm{EQE}$ enhancement above $0.5 \mathrm{eV}$ decreases by increasing inter-subband transition photon energy, suggesting smaller interaction between QD confined levels and higher energy GaAs continuum states. A possible explanation of negative $\triangle \mathrm{EQE}$ in Fig. 70 is nonradiative recombination of IR photoexcited carriers at higher energy trap states. ${ }^{214} \mathrm{At}$ the surface of GaAs, some unoccupied trap states are located above the CB edge. ${ }^{241,242}$ The threshold photon energy of $0.36 \mathrm{eV}$ for the negative $\triangle \mathrm{EQE}$ implies the energy gap between QD ground states and higher energy trap states. IR bias irradiation excites QD electrons, and photoexcited electrons can be captured by such trap states. On the other hand, the traps, of course, do not affect the field-assisted tunneling process. Therefore, IR photoexcitation resulted in EQE reduction. IR photons below $0.36 \mathrm{eV}$ cannot excite QD electrons to the higher energy trap states hence no negative $\triangle \mathrm{EQE}$ is obtained.

As discussed in this section, spectrally and/or timeresolved spectroscopies on TSPA processes can reveal underlying physics of photo-carrier dynamics in IBSCs: (i) Steadystate and up-conversion PL can clarify IB material nature without device fabrication. (ii) Thermal- and field-assisted carrier escape suppress the filling of IB and obscure the intersubband transition. (iii) Inter-subband absorption coefficient and photocurrent production are quantitatively evaluated. Typically, currently available conventional IBSCs indicate small signal contribution on TSPA photocurrent; therefore, modulation spectroscopic technique is preferable for investigation. Furthermore, artificially controlled measurement conditions, e.g., low temperature and high density photoexcitation, which is sometimes far from thermal equilibrium of actual operation conditions, can make it visible about the weak TSPA signal contribution. Further investigations by advanced optical spectroscopies would be indispensable to achieve highly efficient IBSCs overcoming Shockley-Queisser limit.

\section{FUTURE DIRECTIONS}

We have reviewed the on-going topics on the thermodynamics of solar energy conversion in IBSCs, the device physics, and the carrier dynamics processes with a particular emphasis on the two-step inter-subband absorption/recombination processes that are paramount to realization of highefficiency IBSCs. Our aim is to identify the issues and factors that need to be explored and challenged both theoretically and experimentally in order to achieve high-efficiency IBSCs.
Significant efforts and a rapid progress have been made in the device physics as well as practical demonstration of high-efficiency IBSCs. These achievements have been possible due to mature and highly uniform III-V and II-VI thinfilm as well as QW and QD nanostructure material growth and processing technologies.

Strong optical absorption and long carrier lifetimes are prerequisite in IBSC materials and we have pointed out the necessity of optimizing these processes in terms of material and cell structure. The difficulty in the IB approach for photovoltaics stems from the dual nature of IB: it must have optimum carrier occupancy such that it can participate in optical absorptions which promote electrons in-to and out-of the IB. If the occupancy in the IB is too low, the second optical transition (from the IB to the $\mathrm{CB}$ ) is strongly bleached. Conversely, if the occupancy is too high, the first optical absorption (from the VB to the IB) is strongly bleached. Current QD, QW, and HMA materials suffer from low occupancy in the IB and need to be further optimized.

For this, the demonstration of QD-based IBSCs is presently undergoing two stages. The first is to develop technology to fabricate high-density QD arrays or superlattice of low defect density and long carrier lifetime. The fabrication of such QD arrays is most commonly achieved by taking advantage of spontaneous self-assembly of coherent 3D islands in lattice-mismatched epitaxy in S-K growth mode. It has been proven that strain-compensated or strain-balanced growth techniques significantly improve the QD material quality and characteristics of solar cells even after stacking of 50-100 QD layers in S-K growth. The second is to realize ideally halffilled IB states to maximize photocurrent generation by TSPA. The doping of IB region, photofilling by light concentration, and photon confinement structures are currently heavily explored. In addition to further improvements in the material quality, the control of absorption matching in IB materials are expected to improve the efficiency of IBSCs in the near future.

We have also reviewed the two emerging approaches for IBSCs: one with the photon-ratchet approach using molecular system and the other is by using DMSs, both of which, in principle, could inhibit carrier relaxation and hence achieve prolonged lifetimes in the excited states. The fundamental efficiency benefit of relaxation is recognized in up-conversion systems that rely upon sequential absorption TSPA. The novelty of photon-ratchet approach is that optical transitions between the RB and VB are configured to be forbidden. The aim of using ferromagnetic DMS on the other is similar but to mimic triplet states in bulk inorganic semiconductors. In such compounds, spin degeneracy of the bands is lifted, opening the way for configurations favorable for the operation of IBSC, where recombination is suppressed by spin selection rules or by low occupancy of states involved in the allowed recombination.

\section{ACKNOWLEDGMENTS}

Y.O., T.S., and N.A. would also like to acknowledge Professor Stanko Tomić of University of Salford, UK, and Dr. Wladyslaw Walukiewicz and Dr. Kin Man Yu of 
Lawrence Berkeley National laboratory, USA for the valuable discussions and collaboration. The authors gratefully acknowledge the funding support from the New Energy and Industrial Technology Development Organization (NEDO), and the Ministry of Economy, Trade and Industry (METI), Japan, and the Engineering and Physical Sciences Research Council (EPSRC) (Ref. No. EP/ K029398/1), UK. Y.O. and J.F.G. would also like to acknowledge support from the LIA-NextPV Program between CNRS, France and RCAST, the University of Tokyo, and the Japan Society for the Promotion of Science (JSPS).

${ }^{1}$ M. Wolf, Proc. IRE 48, 1246 (1960).

${ }^{2}$ S. Kettemann and J. F. Guillemoles, in Proceedings of the 13th European Photovoltaic Solar Energy Conference (1995), pp. 119-121.

${ }^{3}$ A. Luque and A. Martí, Phys. Rev. Lett. 78, 5014 (1997).

${ }^{4}$ M. J. Keevers and M. A. Green, J. Appl. Phys. 75, 4022 (1994).

${ }^{5}$ M. A. Green, Third Generation Photovoltaics: Advanced Solar Electricity Generation (Springer Berlin Heidelberg, 2003).

${ }^{6}$ A. Luque, A. Martí, E. Antolín, and C. Tablero, Physica B 382, 320 (2006).

${ }^{7}$ W. Shockley and H. J. Queisser, J. Appl. Phys. 32, 510 (1961).

${ }^{8}$ B. M. Kayes, H. Nie, R. Twist, S. G. Spruytte, F. Reinhardt, I. C. Kizilyalli, and G. S. Higashi, in Proceedings of the 37th IEEE Photovoltaic Specialists Conference (PVSC) (IEEE, 2011), pp. 000004-000008.

${ }^{9}$ L. C. Hirst and N. J. Ekins-Daukes, Prog. Photovoltaics: Res. Appl. 19, 286 (2011).

${ }^{10}$ P. Würfel, Sol. Energy Mater. Sol. Cells 38, 23 (1995).

${ }^{11}$ S. P. Bremner, M. Y. Levy, and C. B. Honsberg, Appl. Phys. Lett. 92, 171110 (2008).

${ }^{12}$ M. Yoshida, N. J. Ekins-Daukes, D. J. Farrell, and C. C. Phillips, Appl. Phys. Lett. 100, 263902 (2012).

${ }^{13}$ T. Markvart, J. Opt. A: Pure Appl. Opt. 10, 015008 (2008).

${ }^{14}$ T. Trupke, M. A. Green, and P. Würfel, J. Appl. Phys. 92, 4117 (2002).

${ }^{15}$ N. J. Ekins-Daukes and T. W. Schmidt, Appl. Phys. Lett. 93, 063507 (2008).

${ }^{16}$ P. Olsson, C. Domain, and J.-F. Guillemoles, Phys. Rev. Lett. 102, 227204 (2009)

${ }^{17}$ P. Würfel, J. Phys. C: Solid State Phys. 15, 3967 (1982).

${ }^{18}$ A. V. Semichaevsky and H. T. Johnson, Sol. Energy Mater. Sol. Cells 108, 189 (2013)

${ }^{19}$ A. Martí, L. Cuadra, and A. Luque, IEEE Trans. Electron Devices 49, $1632(2002)$

${ }^{20}$ A. S. Lin and J. D. Phillips, IEEE Trans. Electron Devices 56, 3168 (2009).

${ }^{21}$ K. Yoshida, Y. Okada, and N. Sano, Appl. Phys. Lett. 97, 133503 (2010).

${ }^{22}$ K. Yoshida, Y. Okada, and N. Sano, J. Appl. Phys. 112, 084510 (2012).

${ }^{23}$ R. Strandberg and T. W. Reenaas, Prog. Photovoltaics: Res. Appl. 19, 21 (2011).

${ }^{24}$ I. Tobías, A. Luque, and A. Martí, Semicond. Sci. Technol. 26, 014031 (2011).

${ }^{25}$ A. S. Brown, M. A. Green, and R. P. Corkish, Physica E 14, 121 (2002).

${ }^{26}$ T. Nozawa and Y. Arakawa, J. Appl. Phys. 113, 243102 (2013).

${ }^{27}$ R. Strandberg and T. W. Reenaas, J. Appl. Phys. 105, 124512 (2009).

${ }^{28} \mathrm{~J}$. Nelson, The Physics of Solar Cells (Imperial College Press, 2003).

${ }^{29}$ A. Martí, J. L. Balenzategui, and R. F. Reyna, J. Appl. Phys. 82, 4067 (1997).

${ }^{30}$ E. F. Schubert, Light-Emitting Diodes, 2nd ed. (Cambridge University Press, 2006).

${ }^{31}$ T. Inoue, S. Kido, K. Sasayama, T. Kita, and O. Wada, J. Appl. Phys. 108, 063524 (2010)

${ }^{32}$ A. S. Lin, W. Wang, and J. D. Phillips, J. Appl. Phys. 105, 064512 (2009).

${ }^{33}$ D. Sato, J. Ota, K. Nishikawa, Y. Takeda, N. Miyashita, and Y. Okada, J. Appl. Phys. 112, 094305 (2012).

${ }^{34}$ S. A. Blokhin, A. V. Sakharov, A. M. Nadtochy, A. S. Pauysov, M. V. Maximov, N. N. Ledentsov, A. R. Kovsh, S. S. Mikhrin, V. M. Lantratov, S. A. Mintairov, N. A. Kaluzhniy, and M. Z. Shvarts, Semiconductors 43, 514 (2009).
${ }^{35}$ D. G. Sellers, S. Polly, S. M. Hubbard, and M. F. Doty, Appl. Phys. Lett. 104, 223903 (2014).

${ }^{36}$ S. Asahi, H. Teranishi, N. Kasamatsu, T. Kada, T. Kaizu, and T. Kita, J. Appl. Phys. 116, 063510 (2014).

${ }^{37}$ A. Martí, E. Antolín, E. Cánovas, N. López, P. G. Linares, A. Luque, C. R. Stanley, and C. D. Farmer, Thin Solid Films 516, 6716 (2008).

${ }^{38}$ Y. Y. Cheng, B. Fückel, T. Khoury, R. G. C. R. Clady, M. J. Y. Tayebjee, N. J. Ekins-Daukes, M. J. Crossley, and T. W. Schmidt, J. Phys. Chem. Lett. 1, 1795 (2010).

${ }^{39}$ R. B. Piper, M. Yoshida, D. J. Farrell, T. Khoury, M. J. Crossley, T. W. Schmidt, S. A. Haque, and N. J. Ekins-Daukes, RSC Adv. 4, 8059 (2014).

${ }^{40}$ Dilute III-V Nitride Semiconductors and Materials Systems, edited by A. Erol (Springer, Berlin, Heidelberg, 2008)

${ }^{41}$ S. R. Kurtz, A. A. Allerman, E. D. Jones, J. M. Gee, J. J. Banas, and B. E. Hammons, Appl. Phys. Lett. 74, 729 (1999).

${ }^{42}$ M. Wiemer, V. Sabnis, and H. Yuen, Proc. SPIE 8108, 810804 (2011).

${ }^{43}$ M. Cardona, Phys. Rev. 129, 69 (1963).

${ }^{44}$ M. Kondow, K. Uomi, K. Hosomi, and T. Mozume, Jpn. J. Appl. Phys., Part 2 33, L1056 (1994).

${ }^{45}$ H. J. Lee, L. Y. Juravel, J. C. Woolley, and A. J. S. Thorpe, Phys. Rev. B 21, 659 (1980).

${ }^{46}$ J. Neugebauer and C. G. V. de Walle, Phys. Rev. B 51, 10568 (1995).

${ }^{47}$ L. Bellaiche, S.-H. Wei, and A. Zunger, Phys. Rev. B 54, 17568 (1996).

${ }^{48}$ L. Bellaiche, S.-H. Wei, and A. Zunger, Appl. Phys. Lett. 70, 3558 (1997).

${ }^{49}$ W. G. Bi and C. W. Tu, Appl. Phys. Lett. 70, 1608 (1997).

${ }^{50}$ J. D. Perkins, A. Mascarenhas, Y. Zhang, J. F. Geisz, D. J. Friedman, J. M. Olson, and S. R. Kurtz, Phys. Rev. Lett. 82, 3312 (1999).

${ }^{51}$ W. Shan, W. Walukiewicz, J. W. Ager, E. E. Haller, J. F. Geisz, D. J. Friedman, J. M. Olson, and S. R. Kurtz, J. Appl. Phys. 86, 2349 (1999).

${ }^{52}$ K. M. Yu, W. Walukiewicz, J. W. Ager, D. Bour, R. Farshchi, O. D. Dubon, S. X. Li, I. D. Sharp, and E. E. Haller, Appl. Phys. Lett. 88, 092110 (2006).

${ }^{53}$ K. Alberi, J. Wu, W. Walukiewicz, K. M. Yu, O. D. Dubon, S. P. Watkins, C. X. Wang, X. Liu, Y.-J. Cho, and J. Furdyna, Phys. Rev. B 75, 045203 (2007).

${ }^{54}$ K. M. Yu, W. Walukiewicz, J. Wu, W. Shan, J. W. Beeman, M. A. Scarpulla, O. D. Dubon, and P. Becla, Phys. Rev. Lett. 91, 246403 (2003).

${ }^{55}$ J. Wu, W. Walukiewicz, K. M. Yu, J. W. Ager, E. E. Haller, I. Miotkowski, A. K. Ramdas, C. H. Su, I. K. Sou, R. C. C. Perera, and J. D. Denlinger, Phys. Rev. B 67, 035207 (2003).

${ }^{56}$ K. Alberi, J. Blacksberg, L. D. Bell, S. Nikzad, K. M. Yu, O. D. Dubon, and W. Walukiewicz, Phys. Rev. B 77, 073202 (2008).

${ }^{57}$ W. Walukiewicz, W. Shan, K. M. Yu, J. W. Ager, E. E. Haller, I. Miotkowski, M. J. Seong, H. Alawadhi, and A. K. Ramdas, Phys. Rev. Lett. 85, 1552 (2000).

${ }^{58}$ W. Shan, K. M. Yu, W. Walukiewicz, J. W. Ager, E. E. Haller, and M. C. Ridgway, Appl. Phys. Lett. 75, 1410 (1999).

${ }^{59}$ K. Alberi, K. M. Yu, P. R. Stone, O. D. Dubon, W. Walukiewicz, T. Wojtowicz, X. Liu, and J. K. Furdyna, Phys. Rev. B 78, 075201 (2008).

${ }^{60}$ J. Wu, W. Walukiewicz, and E. E. Haller, Phys. Rev. B 65, 233210 (2002).

${ }^{61}$ P. W. Anderson, Phys. Rev. 124, 41 (1961).

${ }^{62}$ W. Shan, W. Walukiewicz, J. W. Ager, E. E. Haller, J. F. Geisz, D. J. Friedman, J. M. Olson, and S. R. Kurtz, Phys. Rev. Lett. 82, 1221 (1999).

${ }^{63}$ W. Shan, K. M. Yu, W. Walukiewicz, J. Wu, J. W. Ager, and E. E. Haller, J. Phys.: Condens. Matter 16, S3355 (2004).

${ }^{64}$ T. Tanaka, K. M. Yu, A. X. Levander, O. D. Dubon, L. A. Reichertz, N. Lopez, M. Nishio, and W. Walukiewicz, Jpn. J. Appl. Phys., Part 1 50, 082304 (2011).

${ }^{65}$ T. Tanaka, S. Kusaba, T. Mochinaga, K. Saito, Q. Guo, M. Nishio, K. M. Yu, and W. Walukiewicz, Appl. Phys. Lett. 100, 011905 (2012).

${ }^{66}$ W. Shan, W. Walukiewicz, J. W. Ager, K. M. Yu, J. Wu, E. E. Haller, Y. Nabetani, T. Mukawa, Y. Ito, and T. Matsumoto, Appl. Phys. Lett. 83, 299 (2003).

${ }^{67}$ T. Mattila, S.-H. Wei, and A. Zunger, Phys. Rev. B 60, R11245 (1999).

${ }^{68}$ Y. Zhang, A. Mascarenhas, H. P. Xin, and C. W. Tu, Phys. Rev. B 63, 161303 (2001).

${ }^{69}$ P. R. C. Kent, L. Bellaiche, and A. Zunger, Semicond. Sci. Technol. 17, 851 (2002).

${ }^{70}$ P. J. Klar, H. Grüning, W. Heimbrodt, G. Weiser, J. Koch, K. Volz, W. Stolz, S. W. Koch, S. Tomić, S. A. Choulis, T. J. C. Hosea, E. P. O'Reilly, M. Hofmann, J. Hader, and J. V. Moloney, Semicond. Sci. Technol. 17, 830 (2002). 
${ }^{71}$ M. F. Pereira, Jr. and S. Tomić, Appl. Phys. Lett. 98, 061101 (2011).

${ }^{72}$ B. Gu, N. H. Kwong, and R. Binder, Phys. Rev. B 87, 125301 (2013).

${ }^{73}$ B. Lee and L.-W. Wang, Appl. Phys. Lett. 96, 071903 (2010).

${ }^{74}$ C. Tablero, A. Martí, and A. Luque, Appl. Phys. Lett. 96, 121104 (2010).

${ }^{75}$ I. H. Ho and G. B. Stringfellow, MRS Proceedings Material Research Society 449, 871 (1996).

${ }^{76}$ M. Takahashi, A. Moto, S. Tanaka, T. Tanabe, S. Takagishi, K. Karatani, M. Nakayama, K. Matsuda, and T. Saiki, J. Cryst. Growth 221, 461 (2000).

${ }^{77}$ F. Dimroth, A. Howard, J. K. Shurtleff, and G. B. Stringfellow, J. Appl. Phys. 91, 3687 (2002).

${ }^{78}$ J. S. Harris, Jr., J. Cryst. Growth 278, 3 (2005).

${ }^{79}$ N. Miyashita, N. Ahsan, and Y. Okada, Sol. Energy Mater. Sol. Cells 111, 127 (2013)

${ }^{80}$ Y. Jin, R. M. Jock, H. Cheng, Y. He, A. M. Mintarov, Y. Wang, C. Kurdak, J. L. Merz, and R. S. Goldman, Appl. Phys. Lett. 95, 062109 (2009)

${ }^{81}$ P. Krispin, V. Gambin, J. S. Harris, and K. H. Ploog, J. Appl. Phys. 93, 6095 (2003)

${ }^{82}$ S. B. Zhang and S.-H. Wei, Phys. Rev. Lett. 86, 1789 (2001).

${ }^{83}$ N. López, L. A. Reichertz, K. M. Yu, K. Campman, and W. Walukiewicz, Phys. Rev. Lett. 106, 028701 (2011).

${ }^{84}$ A. Luque and A. Martí, Nat. Photonics 5, 137 (2011).

${ }^{85}$ N. Ahsan, N. Miyashita, M. M. Islam, K. M. Yu, W. Walukiewicz, and Y. Okada, Appl. Phys. Lett. 100, 172111 (2012).

${ }^{86}$ M. Burgelman, P. Nollet, and S. Degrave, Thin Solid Films 361-362, 527 (2000).

${ }^{87}$ R. Kudrawiec, G. Sȩk, J. Misiewicz, F. Ishikawa, A. Trampert, and K. H. Ploog, Appl. Phys. Lett. 94, 011907 (2009).

${ }^{88}$ A. Luque and A. Martí, Prog. Photovoltaics: Res. Appl. 9, 73 (2001).

${ }^{89}$ L. Cuadra, A. Martí, and A. Luque, Thin Solid Films 451-452, 593 (2004)

${ }^{90}$ N. Ahsan, N. Miyashita, K. M. Yu, W. Walukiewicz, and Y. Okada, "Electron barrier engineering in a thin-film intermediate band solar cell," IEEE J. Photovoltaics (published online).

${ }^{91}$ R. R. King, D. C. Law, K. M. Edmondson, C. M. Fetzer, G. S. Kinsey, H. Yoon, R. A. Sherif, and N. H. Karam, Appl. Phys. Lett. 90, 183516 (2007).

${ }^{92}$ H. Fujii, K. Watanabe, M. Sugiyama, and Y. Nakano, Jpn. J. Appl. Phys., Part 1 51, 10ND04 (2012).

${ }^{93}$ N. Miyashita, N. Ahsan, and Y. Okada, "Generation and collection of photo-carriers in dilute nitride GaInNAsSb solar cells," Prog. Photovoltaics: Res. Appl. (submitted).

${ }^{94}$ M. M. Islam, N. Miyashita, N. Ahsan, and Y. Okada, Appl. Phys. Lett. 102, 074104 (2013).

${ }^{95}$ N. Ahsan, N. Miyashita, M. M. Islam, K. M. Yu, W. Walukiewicz, and Y. Okada, IEEE J. Photovoltaics 3, 730 (2013).

${ }^{96}$ M. Sydor, N. Jahren, W. C. Mitchel, W. V. Lampert, T. W. Haas, M. Y. Yen, S. M. Mudare, and D. H. Tomich, J. Appl. Phys. 67, 7423 (1990).

${ }^{97}$ W. Wang, A. S. Lin, and J. D. Phillips, Appl. Phys. Lett. 95, 011103 (2009).

${ }^{98}$ T. Tanaka, M. Miyabara, Y. Nagao, K. Saito, Q. Guo, M. Nishio, K. M. Yu, and W. Walukiewicz, IEEE J. Photovoltaics 4, 196 (2014).

${ }^{99}$ T. Tanaka, M. Miyabara, Y. Nagao, K. Saito, Q. Guo, M. Nishio, K. M. Yu, and W. Walukiewicz, Appl. Phys. Lett. 102, 052111 (2013).

${ }^{100}$ J. Phillips, A. Teran, C. Chen, E. Antolín, I. Ramiro, E. López, E. Hernández, I. Artacho, C. Tablero, A. Martí, and A. Luque, in Proceedings of the 39th IEEE Photovoltaic Specialists Conference (PVSC) (2013), p. 1640.

${ }^{101}$ Y. J. Kuang, K. M. Yu, R. Kudrawiec, A. V. Luce, M. Ting, W. Walukiewicz, and C. W. Tu, Appl. Phys. Lett. 102, 112105 (2013).

${ }^{102}$ R. Kudrawiec, A. V. Luce, M. Gladysiewicz, M. Ting, Y. J. Kuang, C. W. Tu, O. D. Dubon, K. M. Yu, and W. Walukiewicz, Phys. Rev. Appl. 1, 034007 (2014).

${ }^{103}$ A. J. Nozik, Physica E 14, 115 (2002).

${ }^{104}$ A. Martí, N. López, E. Antolín, E. Cánovas, C. Stanley, C. Farmer, L. Cuadra, and A. Luque, Thin Solid Films 511-512, 638 (2006)

${ }^{105}$ A. Luque, A. Martí, N. López, E. Antolín, E. Cánovas, C. Stanley, C. Farmer, and P. Díaz, J. Appl. Phys. 99, 094503 (2006).

${ }^{106}$ A. Martí, N. López, E. Antolín, E. Cánovas, A. Luque, C. R. Stanley, C. D. Farmer, and P. Díaz, Appl. Phys. Lett. 90, 233510 (2007).

${ }^{107}$ R. B. Laghumavarapu, M. El-Emawy, N. Nuntawong, A. Moscho, L. F. Lester, and D. L. Huffaker, Appl. Phys. Lett. 91, 243115 (2007).

${ }^{108}$ S. M. Hubbard, C. D. Cress, C. G. Bailey, R. P. Raffaelle, S. G. Bailey, and D. M. Wilt, Appl. Phys. Lett. 92, 123512 (2008).
${ }^{109}$ R. Oshima, A. Takata, and Y. Okada, Appl. Phys. Lett. 93, 083111 (2008).

${ }^{110}$ D. Alonso-Álvarez, A. G. Taboada, J. M. Ripalda, B. Alén, Y. González, L. González, J. M. García, F. Briones, A. Martí, A. Luque, A. M. Sánchez, and S. I. Molina, Appl. Phys. Lett. 93, 123114 (2008).

${ }^{111}$ V. Popescu, G. Bester, M. C. Hanna, A. G. Norman, and A. Zunger, Phys. Rev. B 78, 205321 (2008).

${ }^{112}$ Y. Okada, R. Oshima, and A. Takata, J. Appl. Phys. 106, 024306 (2009).

${ }^{113}$ R. Oshima, A. Takata, Y. Shoji, K. Akahane, and Y. Okada, Physica E 42, 2757 (2010).

${ }^{114}$ S. M. Hubbard, C. Plourde, Z. Bittner, C. G. Bailey, M. Harris, T. Bald, M. Bennett, D. V. Forbes, and R. Raffaelle, in Proceedings of the 35th IEEE Photovoltaic Specialists Conference (PVSC) (IEEE, 2010), pp. 001217-001222.

${ }^{115}$ D. Zhou, G. Sharma, S. F. Thomassen, T. W. Reenaas, and B. O. Fimland, Appl. Phys. Lett. 96, 061913 (2010).

${ }^{116}$ D. Zhou, P. E. Vullum, G. Sharma, S. F. Thomassen, R. Holmestad, T. W. Reenaas, and B. O. Fimland, Appl. Phys. Lett. 96, 083108 (2010).

${ }^{117}$ D. Guimard, R. Morihara, D. Bordel, K. Tanabe, Y. Wakayama, M. Nishioka, and Y. Arakawa, Appl. Phys. Lett. 96, 203507 (2010).

${ }^{118}$ E. Antolín, A. Martí, C. D. Farmer, P. G. Linares, E. Hernández, A. M. Sánchez, T. Ben, S. I. Molina, C. R. Stanley, and A. Luque, J. Appl. Phys. 108, 064513 (2010).

${ }^{119}$ K. A. Sablon, J. W. Little, K. A. Olver, Z. M. Wang, V. G. Dorogan, Y. I. Mazur, G. J. Salamo, and F. J. Towner, J. Appl. Phys. 108, 074305 (2010).

${ }^{120}$ G. Jolley, H. F. Lu, L. Fu, H. H. Tan, and C. Jagadish, Appl. Phys. Lett. 97, 123505 (2010).

${ }^{121}$ V. M. Lantratov, S. A. Mintairov, S. A. Blokhin, N. A. Kalyuzhnyy, N. N. Ledentsov, M. V. Maximov, A. M. Nadtochiy, A. S. Pauysov, A. V. Sakharov, and M. Z. Shvarts, Adv. Sci. Technol. 74, 231 (2010).

${ }^{122}$ T. Sugaya, T. Amano, M. Mori, S. Niki, and M. Kondo, Jpn. J. Appl. Phys., Part 1 49, 030211 (2010).

${ }^{123}$ T. Sugaya, T. Amano, M. Mori, and S. Niki, J. Vac. Sci. Technol., B 28, C3C4 (2010)

${ }^{124}$ Y. Okada, T. Morioka, K. Yoshida, R. Oshima, Y. Shoji, T. Inoue, and T. Kita, J. Appl. Phys. 109, 024301 (2011).

${ }^{125}$ C. G. Bailey, D. V. Forbes, R. P. Raffaelle, and S. M. Hubbard, Appl. Phys. Lett. 98, 163105 (2011).

${ }^{126}$ K. Y. Chuang, T. E. Tzeng, Y. C. Liu, K. D. Tzeng, and T. S. Lay, J. Cryst. Growth 323, 508 (2011).

${ }^{127}$ T. Sugaya, O. Numakami, R. Oshima, S. Furue, H. Komaki, T. Amano, K. Matsubara, Y. Okano, and S. Niki, Energy Environ. Sci. 5, 6233 (2012).

${ }^{128}$ Y. Shoji, K. Akimoto, and Y. Okada, in Proceedings of the 38th IEEE Photovoltaic Specialists Conference (PVSC) (IEEE, 2012), Vol. 2, pp. $1-4$.

${ }^{129}$ C. G. Bailey, D. V. Forbes, S. J. Polly, Z. S. Bittner, Y. Dai, C. Mackos, R. P. Raffaelle, and S. M. Hubbard, IEEE J. Photovoltaics 2, 269 (2012).

${ }^{130}$ K. Tanabe, K. Watanabe, and Y. Arakawa, Appl. Phys. Lett. 100, 192102 (2012).

${ }^{131}$ K. Tanabe, D. Guimard, D. Bordel, and Y. Arakawa, Appl. Phys. Lett. 100, 193905 (2012).

${ }^{132}$ W.-S. Liu, H.-M. Wu, F.-H. Tsao, T.-L. Hsu, and J.-I. Chyi, Sol. Energy Mater. Sol. Cells 105, 237 (2012).

${ }^{133}$ P. J. Carrington, M. C. Wagener, J. R. Botha, A. M. Sanchez, and A. Krier, Appl. Phys. Lett. 101, 231101 (2012).

${ }^{134}$ P. J. Carrington, A. S. Mahajumi, M. C. Wagener, J. R. Botha, Q. Zhuang, and A. Krier, Physica B 407, 1493 (2012).

${ }^{135}$ Y. Eguchi, M. Shiokawa, K. Sakamoto, and K. Yamaguchi, in Proceedings of the 38th IEEE Photovoltaic Specialists Conference (PVSC) (IEEE, 2012), pp. 000045-000047.

${ }^{136}$ M. Shiokawa, E. Saputra, K. Sakamoto, and K. Yamaguchi, in Proceedings of the 39th IEEE Photovoltaic Specialists Conference (PVSC) (IEEE, 2013), pp. 0311-0313.

${ }^{137}$ Y. Shoji, K. Akimoto, and Y. Okada, J. Phys. D: Appl. Phys. 46, 024002 (2013).

${ }^{138}$ F. K. Tutu, J. Wu, P. Lam, M. Tang, N. Miyashita, Y. Okada, J. Wilson, R. Allison, and H. Liu, Appl. Phys. Lett. 103, 043901 (2013).

${ }^{139}$ S. Hatch, J. Wu, K. Sablon, P. Lam, M. Tang, Q. Jiang, and H. Liu, Opt. Express 22, A679 (2014).

${ }^{140}$ J. Hwang, K. Lee, A. Teran, S. Forrest, J. D. Phillips, A. J. Martin, and J. Millunchick, Phys. Rev. Appl. 1, 051003 (2014).

${ }^{141}$ Y. Okada, K. Yoshida, Y. Shoji, and T. Sogabe, IEICE Electron. Express 10, 20132007 (2013). 
${ }^{142}$ S. Tomić, T. S. Jones, and N. M. Harrison, Appl. Phys. Lett. 93, 263105 (2008).

${ }^{143}$ R. Heitz, T. R. Ramachandran, A. Kalburge, Q. Xie, I. Mukhametzhanov, P. Chen, and A. Madhukar, Phys. Rev. Lett. 78, 4071 (1997).

${ }^{144}$ K. Akahane, T. Kawamura, K. Okino, H. Koyama, S. Lan, Y. Okada, M. Kawabe, and M. Tosa, Appl. Phys. Lett. 73, 3411 (1998).

${ }^{145}$ S.-K. Park, J. Tatebayashi, and Y. Arakawa, Appl. Phys. Lett. 84, 1877 (2004)

${ }^{146}$ K. Yamaguchi and T. Kanto, J. Cryst. Growth 275, e2269 (2005).

${ }^{147}$ N. Kakuda, T. Yoshida, and K. Yamaguchi, Appl. Surf. Sci. 254, 8050 (2008).

${ }^{148}$ E. Saputra, J. Ohta, N. Kakuda, and K. Yamaguchi, Appl. Phys. Express 5, 125502 (2012).

${ }^{149}$ Z. R. Wasilewski, S. Fafard, and J. P. McCaffrey, J. Cryst. Growth 201-202, 1131 (1999).

${ }^{150}$ T. K. Woodward, T. Sizer, D. L. Sivco, and A. Y. Cho, Appl. Phys. Lett. 57, 548 (1990).

${ }^{151}$ N. J. Ekins-Daukes, K. W. J. Barnham, J. P. Connolly, J. S. Roberts, J. C. Clark, G. Hill, and M. Mazzer, Appl. Phys. Lett. 75, 4195 (1999).

${ }^{152}$ K. Akahane, N. Yamamoto, and T. Kawanishi, Phys. Status Solidi A 208, 425 (2011).

${ }^{153}$ C. G. Bailey, S. M. Hubbard, D. V. Forbes, and R. P. Raffaelle, Appl Phys. Lett. 95, 203110 (2009).

${ }^{154}$ C. G. Bailey, S. J. Polly, J. Okvath, D. V. Forbes, C. D. Cress, S. M. Hubbard, and R. P. Raffaelle, in Proceedings of the 35th IEEE Photovoltaic Specialists Conference (PVSC) (IEEE, 2010), pp. 000364-000369.

${ }^{155}$ M. Y. Levy and C. Honsberg, IEEE Trans. Electron Devices 55, 706 (2008).

${ }^{156}$ S. Tomić, Appl. Phys. Lett. 103, 072112 (2013).

${ }^{157}$ R. Heitz, M. Veit, N. N. Ledentsov, A. Hoffmann, D. Bimberg, V. M. Ustinov, P. S. Kop'ev, and Z. I. Alferov, Phys. Rev. B 56, 10435 (1997).

${ }^{158}$ Y. D. Jang, H. Lee, D. Lee, J. S. Kim, J. Y. Leem, and S. K. Noh, J. Appl. Phys. 99, 096101 (2006).

${ }^{159}$ R. Teissier, D. Sicault, J. C. Harmand, G. Ungaro, G. L. Roux, and L. Largeau, J. Appl. Phys. 89, 5473 (2001).

${ }^{160}$ J.-B. Wang, S. R. Johnson, S. A. Chaparro, D. Ding, Y. Cao, Y. G. Sadofyev, Y.-H. Zhang, J. A. Gupta, and C. Z. Guo, Phys. Rev. B 70, 195339 (2004)

${ }^{161}$ W.-H. Chang, Y.-A. Liao, W.-T. Hsu, M.-C. Lee, P.-C. Chiu, and J.-I. Chyi, Appl. Phys. Lett. 93, 033107 (2008).

${ }^{162}$ Y. S. Chiu, M. H. Ya, W. S. Su, and Y. F. Chen, J. Appl. Phys. 92, 5810 (2002).

${ }^{163}$ K. Nishikawa, Y. Takeda, K. Yamanaka, T. Motohiro, D. Sato, J. Ota, N. Miyashita, and Y. Okada, J. Appl. Phys. 111, 044325 (2012).

${ }^{164}$ K. Nishikawa, Y. Takeda, T. Motohiro, D. Sato, J. Ota, N. Miyashita, and Y. Okada, Appl. Phys. Lett. 100, 113105 (2012).

${ }^{165}$ O. B. Shchekin and D. G. Deppe, Appl. Phys. Lett. 80, 2758 (2002).

${ }^{166} \mathrm{O}$. Wada, A. Suzuki, Y. Ogawa, and K. Tajima, in Femtosecond Technology, edited by T. Kamiya, F. Saito, O. Wada, and H. Yajima (Springer, Berlin, Heidelberg, 1999), p. 59.

${ }^{167}$ R. Dingle, H. L. Störmer, A. C. Gossard, and W. Wiegmann, Appl. Phys. Lett. 33, 665 (1978).

${ }^{168}$ J. Phillips, K. Kamath, X. Zhou, N. Chervela, and P. Bhattacharya, Appl. Phys. Lett. 71, 2079 (1997).

${ }^{169}$ J. S. Kim, P. W. Yu, J.-Y. Leem, J. I. Lee, S. K. Noh, J. S. Kim, G. H. Kim, S.-K. Kang, S. I. Ban, S. G. Kim, Y. D. Jang, U. H. Lee, J. S. Yim, and D. Lee, J. Cryst. Growth 234, 105 (2002).

${ }^{170}$ T. Kita, R. Hasagawa, and T. Inoue, J. Appl. Phys. 110, 103511 (2011).

${ }^{171}$ A. Martí, L. Cuadra, and A. Luque, IEEE Trans. Electron Devices 48, $2394(2001)$

${ }^{172}$ A. Martí, E. Antolín, C. R. Stanley, C. D. Farmer, N. López, P. Díaz, E. Cánovas, P. G. Linares, and A. Luque, Phys. Rev. Lett. 97, 247701 (2006).

${ }^{173}$ K. A. Sablon, J. W. Little, V. Mitin, A. Sergeev, N. Vagidov, and K. Reinhardt, Nano Lett. 11, 2311 (2011).

${ }^{174}$ X. Yang, K. Wang, Y. Gu, H. Ni, X. Wang, T. Yang, and Z. Wang, Sol. Energy Mater. Sol. Cells 113, 144 (2013).

${ }^{175}$ P. Lam, S. Hatch, J. Wu, M. Tang, V. G. Dorogan, Y. I. Mazur, G. J. Salamob, I. Ramiro, A. Seeds, and H. Liu, Nano Energy 6, 159 (2014).

${ }^{176}$ A. Luque, A. Martí, and C. Stanley, Nat. Photonics 6, 146 (2012).

${ }^{177}$ K. Sakamoto, Y. Kondo, K. Uchida, and K. Yamaguchi, J. Appl. Phys. 112, 124515 (2012).

${ }^{178}$ I. Tobías, A. Luque, E. Antolín, P. García-Linares, I. Ramiro, E. Hernández, and A. Martí, J. Appl. Phys. 112, 124518 (2012).

${ }^{179}$ S. M. Hubbard, C. G. Bailey, R. Aguinaldo, S. Polly, D. V. Forbes, and R. P. Raffaelle, in Proceedings of the 34th IEEE Photovoltaic Specialists Conference (PVSC) (IEEE, 2009), pp. 000090-000095.
${ }^{180}$ M. Yang and M. Yamaguchi, Sol. Energy Mater. Sol. Cells 60, 19 (2000).

${ }^{181}$ A. Takata, R. Oshima, Y. Shoji, K. Akahane, and Y. Okada, in Proceedings of the 35th IEEE Photovoltaic Specialists Conference (PVSC) (IEEE, 2010), pp. 001877-001880.

${ }^{182}$ P. G. Linares, A. Martí, E. Antolín, C. D. Farmer, I. Ramiro, C. R. Stanley, and A. Luque, Sol. Energy Mater. Sol. Cells 98, 240 (2012).

${ }^{183}$ T. Sogabe, Y. Shoji, M. Ohba, K. Yoshida, R. Tamaki, H.-F. Hong, C.-H. Wu, C.-T. Kuo, S. Tomić, and Y. Okada, Sci. Rep. 4, 4792 (2014).

${ }^{184}$ Y. Harada, T. Maeda, and T. Kita, J. Appl. Phys. 113, 223511 (2013).

${ }^{185}$ D. Birkedal, J. Bloch, J. Shah, L. N. Pfeiffer, and K. West, Appl. Phys. Lett. 77, 2201 (2000).

${ }^{186}$ T. Dietl, H. Ohno, F. Matsukura, J. Cibert, and D. Ferrand, Science 287, 1019 (2000).

${ }^{187}$ J.-M. Raulot, C. Domain, and J.-F. Guillemoles, Phys. Rev. B 71, 035203 (2005).

${ }^{188}$ K. Emery, Handbook of Photovoltaic Science and Engineering, 2nd ed., edited by A. H. Luque and S. Hegedus (John Wiley \& Sons, New York, 2010).

${ }^{189}$ E. Antolín, A. Martí, J. Olea, D. Pastor, G. González-Díaz, I. Mártil, and A. Luque, Appl. Phys. Lett. 94, 042115 (2009).

${ }^{190}$ K. Sánchez, I. Aguilera, P. Palacios, and P. Wahnón, Phys. Rev. B 82, 165201 (2010).

${ }^{191}$ J. J. Krich, B. I. Halperin, and A. Aspuru-Guzik, J. Appl. Phys. 112, 013707 (2012)

${ }^{192}$ L. Hedin, Phys. Rev. 139, A796 (1965).

${ }^{193}$ M. Städele, J. A. Majewski, P. Vogl, and A. Görling, Phys. Rev. Lett. 79, 2089 (1997).

${ }^{194}$ T. C. Schulthess, W. M. Temmerman, Z. Szotek, W. H. Butler, and G. M. Stocks, Nat. Mater. 4, 838 (2005)

${ }^{195}$ T. Dietl, F. Matsukura, and H. Ohno, Phys. Rev. B 66, 033203 (2002).

${ }^{196}$ P. Mahadevan and A. Zunger, Phys. Rev. B 69, 115211 (2004).

${ }^{197}$ P. Wahnón and C. Tablero, Phys. Rev. B 65, 165115 (2002).

${ }^{198}$ C. Tablero, Sol. Energy Mater. Sol. Cells 90, 588 (2006).

${ }^{199}$ Y. Seminóvski, P. Palacios, and P. Wahnón, Thin Solid Films 519, 7517 (2011).

${ }^{200}$ C. Tablero, A. J. García, J. J. Fernández, P. Palacios, and P. Wahnón, Comput. Mater. Sci. 27, 58 (2003)

${ }^{201}$ I. Aguilera, P. Palacios, and P. Wahnón, Thin Solid Films 516, 7055 (2008).

${ }^{202}$ I. Aguilera, P. Palacios, K. Sánchez, and P. Wahnón, Phys. Rev. B 81, 075206 (2010).

${ }^{203}$ R. Fiederling, M. Keim, G. Reuscher, W. Ossau, G. Schmidt, A. Waag, and L. W. Molenkamp, Nature 402, 787 (1999).

${ }^{204}$ A. Janotti and S.-H. Wei, Appl. Phys. Lett. 81, 3957 (2002).

${ }^{205}$ J.-F. Guillemoles, J. M. Raulot, and C. Domain, in 17th Workshop on Quantum Solar Energy Conversion, European Society for Quantum Solar Energy Conversion, 2005.

${ }^{206}$ J.-F. Guillemoles, P. Olsson, and C. Domain, in 19th Workshop on Quantum Solar Energy Conversion, European Society for Quantum Solar Energy Conversion, 2007.

${ }^{207}$ B. Sanyal, O. Bengone, and S. Mirbt, Phys. Rev. B 68, 205210 (2003).

${ }^{208}$ M. E. Overberg, G. T. Thaler, R. M. Frazier, C. R. Abernathy, S. J. Pearton, R. Rairigh, J. Kelly, N. A. Theodoropoulou, A. F. Hebard, R. G. Wilson, and J. M. Zavada, Electrochem. Solid-State Lett. 7, G44 (2004).

${ }^{209}$ M. E. Overberg, G. T. Thaler, R. M. Frazier, R. Rairigh, J. Kelly, C. R. Abernathy, S. J. Pearton, A. F. Hebard, R. G. Wilson, and J. M. Zavada, Solid-State Electron. 47, 1549 (2003).

${ }^{210}$ N. J. Ekins-Daukes, C. B. Honsberg, and M. Yamaguchi, in Conference Record of the Thirty-First IEEE Photovoltaic Specialists Conference (IEEE, 2005), pp. 49-54.

${ }^{211}$ Y. Shoji, K. Akimoto, and Y. Okada, J. Appl. Phys. 112, 064314 (2012).

${ }^{212}$ Y. Shoji, K. Narahara, H. Tanaka, T. Kita, K. Akimoto, and Y. Okada, J. Appl. Phys. 111, 074305 (2012).

${ }^{213}$ G. Jolley, L. Fu, H. F. Lu, H. H. Tan, and C. Jagadish, Prog. Photovoltaics: Res. Appl. 21, 736 (2013).

${ }^{214}$ R. Tamaki, Y. Shoji, Y. Okada, and K. Miyano, Appl. Phys. Lett. 105, 073118 (2014).

${ }^{215}$ R. Tamaki, Y. Shoji, Y. Okada, and K. Miyano, IEEE J. Photovoltaics 5, 229 (2015).

${ }^{216}$ E. Antolín, A. Martí, C. R. Stanley, C. D. Farmer, E. Cánovas, N. López, P. G. Linares, and A. Luque, Thin Solid Films 516, 6919 (2008).

${ }^{217}$ A. Scaccabarozzi, S. Adorno, S. Bietti, M. Acciarri, and S. Sanguinetti, Phys. Status Solidi RRL 7, 173 (2013). 
${ }^{218}$ Z. S. Bittner, S. Hellstroem, S. J. Polly, R. B. Laghumavarapu, B. Liang, D. L. Huffaker, and S. M. Hubbard, Appl. Phys. Lett. 105, 253903 (2014).

${ }^{219}$ M. Sugiyama, Y. Wang, K. Watanabe, T. Morioka, Y. Okada, and Y. Nakano, IEEE J. Photovoltaics 2, 298 (2012).

${ }^{220}$ M. Sugiyama, Y. Wang, H. Fujii, H. Sodabanlu, K. Watanabe, and Y. Nakano, J. Phys. D: Appl. Phys. 46, 024001 (2013).

${ }^{221}$ M. Yoshida, H. Amrania, D. J. Farrell, B. Browne, E. Yoxall, N. J. EkinsDaukes, and C. C. Phillips, IEEE J. Photovoltaics 4, 634 (2014).

${ }^{222}$ M. Elborg, M. Jo, Y. Ding, T. Noda, T. Mano, and K. Sakoda, Jpn. J. Appl. Phys., Part 1 51, 06FF15 (2012).

${ }^{223}$ D. M. Tex, I. Kamiya, and Y. Kanemitsu, Phys. Rev. B 87, 245305 (2013).

${ }^{224}$ D. M. Tex, I. Kamiya, and Y. Kanemitsu, Sci. Rep. 4, 4125 (2014).

${ }^{225}$ D. M. Tex, T. Ihara, I. Kamiya, and Y. Kanemitsu, Phys. Rev. B 89, 125301 (2014).

${ }^{226}$ J. Wu, D. Shao, Z. Li, M. O. Manasreh, V. P. Kunets, Z. M. Wang, and G. J. Salamo, Appl. Phys. Lett. 95, 071908 (2009).

${ }^{227}$ T. Kita, T. Maeda, and Y. Harada, Phys. Rev. B 86, 035301 (2012).

${ }^{228}$ B. N. Murdin, A. R. Hollingworth, J. A. Barker, D. G. Clarke, P. C. Findlay, C. R. Pidgeon, J.-P. R. Wells, I. V. Bradley, S. Malik, and R. Murray, Phys. Rev. B 62, R7755 (2000).

${ }^{229}$ P. Rale, A. Delamarre, G. El-Hajje, R. Tamaki, K. Watanabe, Y. Shoji, Y. Okada, M. Sugiyama, L. Lombez, and J.-F. Guillemoles, "Quantitative optical measurement of chemical potentials in intermediate band solar cells," J. Photon. Energy (to be published).
${ }^{230}$ G. W. p't Hooft, W. A. J. A. van der Poel, L. W. Molenkamp, and C. T. Foxon, Phys. Rev. B 35, 8281 (1987).

${ }^{231}$ A. Luque, A. Martí, A. Mellor, D. F. Marrón, I. Tobías, and E. Antolín, Prog. Photovoltaics: Res. Appl. 21, 658 (2013).

${ }^{232}$ W. G. Hu, T. Inoue, O. Kojima, and T. Kita, Appl. Phys. Lett. 97, 193106 (2010).

${ }^{233} \mathrm{~S}$. Tomić, T. Sogabe, and Y. Okada, "In-plane coupling effect on absorption coefficients of InAs/GaAs quantum dots arrays for intermediate band solar cell," Prog. Photovoltaics: Res. Appl. (published online 2014).

${ }^{234}$ H. A. Atwater and A. Polman, Nat. Mater. 9, 205 (2010).

${ }^{235}$ A. Polman and H. A. Atwater, Nat. Mater. 11, 174 (2012).

${ }^{236}$ N. Kasamatsu, T. Kada, A. Hasegawa, Y. Harada, and T. Kita, J. Appl. Phys. 115, 083510 (2014).

${ }^{237}$ Y. Ikeuchi, T. Inoue, M. Asada, Y. Harada, T. Kita, E. Taguchi, and H. Yasuda, Appl. Phys. Express 4, 062001 (2011).

${ }^{238}$ A. Takahashi, T. Ueda, Y. Bessho, Y. Harada, T. Kita, E. Taguchi, and H. Yasuda, Phys. Rev. B 87, 235323 (2013).

${ }^{239}$ M. Usman, T. Inoue, Y. Harada, G. Klimeck, and T. Kita, Phys. Rev. B 84, 115321 (2011).

${ }^{240}$ V. Popescu, G. Bester, and A. Zunger, Appl. Phys. Lett. 95, 023108 (2009).

${ }^{241}$ M. Murayama, K. Shiraishi, and T. Nakayama, Jpn. J. Appl. Phys., Part 1 37, 4109 (1998).

${ }^{242}$ T. Kita, O. Wada, T. Nakayama, and M. Murayama, Phys. Rev. B 66, 195312 (2002).

${ }^{243}$ P. Olsson, C. Domain, and J.-F. Guillemoles, paper present at the 19th Quantsol Conference (2007). 